\title{
On the vanishing of Love numbers for Kerr black holes
}

\author{
Panagiotis Charalambous, ${ }^{a}$ Sergei Dubovsky ${ }^{a}$ and Mikhail M. Ivanov ${ }^{a, b}$ \\ ${ }^{a}$ Center for Cosmology and Particle Physics, Department of Physics, New York University, \\ New York, NY 10003, U.S.A. \\ ${ }^{b}$ Institute for Nuclear Research of the Russian Academy of Sciences, \\ 60th October Anniversary Prospect, 7a, 117312 Moscow, Russia \\ E-mail: pc2560@nyu.edu, dubovsky@nyu.edu, mi1271@nyu.edu
}

ABSTRACT: It was shown recently that the static tidal response coefficients, called Love numbers, vanish identically for Kerr black holes in four dimensions. In this work, we confirm this result and extend it to the case of spin- 0 and spin- 1 perturbations. We compute the static response of Kerr black holes to scalar, electromagnetic, and gravitational fields at all orders in black hole spin. We use the unambiguous and gauge-invariant definition of Love numbers and their spin- 0 and spin- 1 analogs as Wilson coefficients of the point particle effective field theory. This definition also allows one to clearly distinguish between conservative and dissipative response contributions. We demonstrate that the behavior of Kerr black hole responses to spin- 0 and spin- 1 fields is very similar to that of the spin-2 perturbations. In particular, static conservative responses vanish identically for spinning black holes. This implies that vanishing Love numbers are a generic property of black holes in four-dimensional general relativity. We also show that the dissipative part of the response does not vanish even for static perturbations due to frame-dragging.

KeYwords: Black Holes, Classical Theories of Gravity, Effective Field Theories

ArXiv EPrint: 2102.08917 


\section{Contents}

1 Introduction $\quad 2$

2 Newtonian tidal response 4

3 Scalar response coefficients $\quad 8$

3.1 Definition from Newtonian matching 9

3.2 Static response in the EFT 9

$\begin{array}{ll}3.3 \text { Microscopic computation } & 17\end{array}$

3.4 Matching to the EFT 21

4 Spin-1 response coefficients $\quad 24$

4.1 Definition 24

4.2 Newman-Penrose formalism 26

4.3 From Maxwell-Newman-Penrose scalars to response coefficients 27

4.4 Matching to the EFT 29

$5 \quad$ Spin-2 response coefficients 31

5.1 Definition 31

5.2 From Weyl scalar to response coefficients 32

5.3 Matching to the EFT 33

6 Master formula for black hole response coefficients $\quad 35$

6.1 Static responses 35

6.2 Time-dependent responses 36

$\begin{array}{lll}7 & \text { Discussion and conclusions } & 39\end{array}$

A Useful mathematical relations $\quad 40$

A.1 Spherical harmonics 40

A.2 Gamma function $\quad 42$

A.3 Gauss hypergeometric function 43

B Calculation of Maxwell-Newman-Penrose scalars 43

C Spin-1 magnetic Love numbers $\quad 44$

$\begin{array}{ll}\text { D Comment on the near-field approximation } & 46\end{array}$

D.1 Scalar field example 46

D.2 Teukolsky equation in the near-field approximation 48

D.3 Comparison with the low-frequency solution 49 


\section{Introduction}

The static response of black holes to external perturbations, captured by the so-called Love numbers, ${ }^{1}$ has recently attracted significant attention both from the observational and theoretical sides. On the one hand, black holes' Love numbers are measurable quantities that can be probed with gravitational wave observations $[2,3]$. On the other hand, they play an important role in the effective field theory (EFT) of binary inspirals [4-7], where they determine Wilson coefficients that describe leading finite-size effects.

The tidal gravitational Love numbers of non-rotating Schwarzschild black holes have been independently computed by Fang and Lovelace [8], Damour and Nagar [9], and by Binnington and Poisson [10]. Remarkably, they vanish in four dimensions in general relativity (GR), which poses a naturalness problem from the EFT point of view [6], and therefore might hint on the existence of a new symmetry of black holes. Intriguingly, there exist several physical examples where the black holes' Love numbers do not vanish. In particular, the calculation of Love numbers has been extended to higher dimensions in refs. [11, 12], which have shown that their identical vanishing for all multipoles is a unique result taking pace only in four dimensions. Recently this result has been generalized to the cases of spin-0, spin-1 and spin-2 perturbations of different parities in ref. [7]: the static responses of Schwarzschild black holes are generally non-zero for all these different types of perturbations, but accidentally they vanish in four dimensions. Moreover, black holes' Love numbers were found to be non-zero in certain modified gravity theories $[3,13,14]$.

As of now, it has been firmly established that the Love numbers of all perturbing fields vanish in four dimensions for Schwarzshield black holes [7-11, 15]. However, the properties of spinning (Kerr) black holes [16] are still under debate. Tidal deformations of slowly rotating black holes were studied in refs. [17-22], which have found that the Love numbers vanish for axisymmetric perturbations. Moreover, Landry and Poisson (2015) [20] have claimed that the Love numbers vanish for other types of perturbations at first order in black hole's spin. However, this result was recently questioned by Le Tiec and Casals [23], who argued that conclusions of Landry and Poisson (2015) might have been affected by an uncertainty introduced by the split of the gravitational potential into the source and response parts. Similar concerns have been earlier raised in the context of Schwarzschild black holes [11, 24]. To avoid that ambiguity, Kol and Smolkin [11] have used an analytic continuation of the relevant general relativity solutions into higher dimensions, which is effectively equivalent to promoting the orbital mode number (multipolar index) $\ell$ to noninteger values. Using a similar analytic continuation technique, Le Tiec et al. (2020) [23, 25] have obtained non-vanishing static response coefficients and have claimed that the Love numbers do not vanish for general spin-2 (tidal) perturbations around Kerr black holes.

Recently, Chia (2020) [26] and Goldberger et al. (2020) [27] have pointed out that the "Love numbers" that Le Tiec et al. (2020) have computed actually correspond to dissipative effects, whereas the conservative tidal response vanishes identically for spinning black holes. Analogous results have also appeared in refs. [22, 28]. All these works imply that the Love

\footnotetext{
${ }^{1}$ The Love numbers are named after the mathematician A.E.H. Love who introduced them to describe the tidal deformation of the Earth in ref. [1].
} 
numbers defined in the classical sense of conservative tidal deformability are zero for Kerr black holes.

In this work, we compute analogs of the tidal Love numbers produced by spin- 0 and spin-1 perturbations around Kerr black holes. We will define Love numbers as Wilson coefficients of local operators in the worldline point-particle effective field theory. This will allow us to distinguish between the conservative response to external fields, which is related to static Love numbers, and the dissipative part of black hole's response. The finite-size local EFT operators are expected to be present in the EFT on general grounds and they should have a tensorial structure dictated by the axial symmetry of the Kerr background. We introduce these couplings in the EFT for static fields and demonstrate how the new tensorial Wilson coefficients are related to the response coefficients that we have extracted from the solutions to linearized spin- 0 , spin- 1 , and spin-2 field perturbations in the Kerr background. We show that the structure of these GR solutions is such that the dissipative parts of scalar and electromagnetic responses do not vanish for the Kerr black holes just like their spin-2 counterparts. However, the EFT Wilson coefficients that capture the local (conservative) responses of spinning black holes vanish for all bosonic perturbing fields.

On the technical side, we demonstrate that the analytic continuation procedure utilized in refs. $[25,26]$ allows one to avoid the uncertainty in the source/response split and obtain consistent gauge-independent results for response coefficients in the spin- 0 and spin- 1 cases. We also give an interpretation of this analytic continuation procedure in the EFT context. We show that the subleading source corrections, which may overlap with the induced response contributions, are, in fact, produced by interactions between external fields and gravitational degrees of freedom. This observation allows one to unambiguously identify the black hole multipole moment induced by external fields. Indeed, the graviton corrections to the source solution can be computed order by order within the EFT. Thus, given a full GR solution, one can subtract the graviton interaction contributions from it and hence robustly extract the Love numbers. This procedure is equivalent to using the analytic continuation $\ell \rightarrow \mathbb{R}$.

Our paper is structured as follows. We start with a recap of the Newtonian response coefficients in section 2. Then we focus on the scalar response coefficients in section 3 , where we discuss in detail their calculation both in general relativity and in the pointparticle EFT. In section 4 we compute static response of the Kerr black hole to the external electric field and match this result with the EFT calculation. We repeat the same procedure for the spin-2 (gravitational) perturbation in section 5. Finally, we recap the main general relativity calculations for all spins in section 6 and extend them to the case of non-static perturbations. We discuss our main results and draw conclusions in section 7. Some additional material is presented in several appendices. Appendix A is a brief reference to key mathematical relations and conventions. In appendix B we give some details on the calculation of the Newman-Penrose-Maxwell scalars, which encapsulate the electromagnetic field around the Kerr black hole and which are required to extract spin1 response coefficients. We explicitly compute the spin-1 magnetic response coefficients in appendix $\mathrm{C}$ - they happen to identically coincide with the electric ones. Finally, in appendix D we comment on the validity of the response coefficients computed in the potential region approximation. 
Conventions. In what follows we will work with the metric with signature $(-,+,+,+)$; greek letters (e.g. $\mu, \nu$, etc.) will denote the spacetime indices; latin letters from the middle of the alphabet (e.g. $i, j$, etc.) will denote the spatial 3-dimensional indices; latin letter from the beginning of the alphabet (e.g. $a, b$, etc.) will run over the coordinates on the two-sphere $\mathbb{S}^{2}$. We will work in the $c=G=1$ units in most of the paper.

\section{Newtonian tidal response}

In this section we review the definition of tidal response in Newtonian theory [10, 25, 29], and discuss some important subtleties present for spinning bodies.

Spherical bodies. Let us consider a non-rotating spherical body of mass $M$ and equilibrium radius $r_{s}$. Now imagine that we adiabatically apply an external gravitational field $U_{\text {ext }}$. It is convenient to place the body in the origin of the coordinate system. Then we can characterize the external source in terms of the multipole moments,

$$
\mathcal{E}_{L}(t)=-\left.\frac{1}{(\ell-2) !} \partial_{\left\langle i_{1}\right.} \ldots \partial_{\left.i_{\ell}\right\rangle} U_{\mathrm{ext}}\right|_{r=0}
$$

where $r$ is the distance from the origin, $\langle\ldots\rangle$ denotes the symmetric trace-free part, and $L \equiv i_{1} \ldots i_{\ell}$ is the multi-index. The multipole moments $\mathcal{E}_{L}$ are symmetric trace-free tensors (STFs) that parametrically depend on time $t$. In what follows we suppress this explicit parametric dependence of the tidal field $\mathcal{E}_{L}$ on time. In response to the external field, the body will deform and develop internal multipole moments $I_{L}$,

$$
I_{L} \equiv \int_{\mathbb{R}^{3}} d^{3} x \rho(\mathbf{x}) x^{\langle L\rangle},
$$

where $\rho(\mathbf{x})$ is perturbed body's density and $x^{L} \equiv x^{i_{1}} \ldots x^{i_{\ell}}$. Summing up the two contributions, we find the following expression for the total Newtonian gravitational potential

$$
U=\frac{M}{r}-\sum_{\ell=2}\left[\frac{(\ell-2) !}{\ell !} \mathcal{E}_{L} x^{L}-\frac{(2 \ell-1) ! !}{\ell !} \frac{I_{L} n^{L}}{r^{\ell+1}}\right],
$$

where $n^{L} \equiv n^{i_{1}} \ldots n^{i_{\ell}}$ is the tensor product of unit direction vectors $n^{i} \equiv x^{i} /|\mathbf{x}|$, and we have omitted the dipole moment $\ell=1$ since it corresponds to trivial center-of-mass translations. At this point, it is convenient to switch to the spherical coordinates and use an expansion of the external source and induced multipole moments in terms of the spherical harmonics $Y_{\ell m}(\theta, \phi)$. This way eq. (2.3) can be rewritten as follows:

$$
U=\frac{M}{r}-\sum_{\ell=2} \sum_{m=-\ell}^{\ell} Y_{\ell m}\left[\frac{(\ell-2) !}{\ell !} \mathcal{E}_{\ell m} r^{\ell}-\frac{(2 \ell-1) ! !}{\ell !} \frac{I_{\ell m}}{r^{\ell+1}}\right],
$$

where the angular harmonic coefficients $\mathcal{E}_{\ell m}$ are related to the STF components via

$$
n^{L} \mathcal{E}_{L}=\sum_{m=-\ell}^{\ell} \mathcal{E}_{\ell m} Y_{\ell m}, \quad \mathcal{E}_{\ell m}=\mathcal{E}_{L} \oint_{\mathbb{S}^{2}} d \Omega n^{L} Y_{\ell m}^{*}
$$

and $d \Omega=\sin \theta d \theta d \phi$ denotes the measure on the two-sphere $\mathbb{S}^{2}$. 
If the external gravitational field is adiabatic and weak, linear response theory dictates that the response multipoles should be proportional to the perturbing multipole moments $[29-33],{ }^{2}$

$$
\begin{aligned}
I_{L}(t) & =\lambda_{\ell} \mathcal{E}_{L}(t)-\nu_{\ell} \frac{d}{d t} \mathcal{E}_{L}(t)+\ldots \approx \lambda_{\ell} \mathcal{E}_{L}\left(t-\tau^{\prime}\right), \quad \text { or } \\
I_{\ell m} & =\lambda_{\ell} \mathcal{E}_{\ell m}-\nu_{\ell} \frac{d}{d t} \mathcal{E}_{\ell m}+\ldots,
\end{aligned}
$$

where we have restored the explicit time-dependence for clarity, and "..." denotes nonlinear corrections and contributions with more time derivatives. Here the coefficient $\lambda_{\ell}$ is referred to as the Newtonian tidal Love number, while $\nu_{\ell}$ is the dissipative response coefficient related to body's kinematic viscosity [29]. This contribution captures the fact that dissipation produces a time lag $\tau^{\prime}$ between the external field and body's response [33]. ${ }^{3}$ Note that non-zero viscosity triggers various dissipative effects, such as heating of the body and the transfer of angular momentum between the body and the source, known as "tidal torque," see e.g. [34]. Using the frequency-space ansatz $\mathcal{E}_{L} \propto e^{-i \omega t}$, eq. (2.6) can be written as

$$
I_{\ell m}=\lambda_{\ell} \mathcal{E}_{\ell m}+i \nu_{\ell} \omega \mathcal{E}_{\ell m}+\ldots
$$

If the external tidal environment is static in body's rest frame, the viscosity contribution disappears.

Spinning bodies. If the test body is rotating, the definition of response coefficients is more intricate. The rotating body will generally depart from spherical symmetry and hence it will have internal multipole moments even in the absence of an external perturbation. For a moment, let us assume that the body's equilibrium configuration can be approximated as a rigidly rotating sphere. If the rotation is sufficiently slow, the linear response in the body's rotation frame takes the same form (2.6) as for the non-rotating body [29]. However, an important effect appears when we switch to an inertial frame. Let us focus on the leading frequency-dependent contribution $\propto \frac{d}{d t} \mathcal{E}$. Because of rotation, the total time derivative in the body's rotation frame takes the following form

$$
\frac{d}{d t} \mathcal{E}_{i_{1} \ldots i_{\ell}}=\frac{\partial}{\partial t} \mathcal{E}_{i_{1} \ldots i_{\ell}}-\sum_{n=1}^{\ell} \Omega_{i_{n} j} \mathcal{E}_{i_{1} \ldots i_{n-1} j i_{n+1} \ldots i_{\ell}} \equiv \frac{\partial}{\partial t} \mathcal{E}_{i_{1} \ldots i_{\ell}}+\kappa_{L}^{L^{\prime}} \mathcal{E}_{L^{\prime}}
$$

where $\frac{\partial}{\partial t} \mathcal{E}$ is the time derivative in a fixed inertial frame. Here we introduced the angular velocity tensor $\Omega_{i j}=-\Omega_{j i}$ and defined

$$
\kappa_{i_{1}^{\prime} \ldots i_{\ell}^{\prime}}^{i_{1} \ldots i_{\ell}} \equiv-\sum_{n=1}^{\ell} \Omega_{i_{n} i_{n}^{\prime}} \delta_{i_{1}^{\prime}}^{i_{1}} \ldots \delta_{i_{n+1}^{\prime}}^{i_{n+1}} \delta_{i_{n-1}^{\prime}}^{i_{n-1}} \ldots \delta_{i_{\ell}^{\prime}}^{i_{\ell}}
$$

\footnotetext{
${ }^{2}$ Note that the coupling between different orbital and azimuthal modes is absent by virtue of linearity, i.e. weakness of the external perturbations.

${ }^{3}$ This is true for small adiabatic perturbations of the body's equilibrium configuration. In the case of weak friction this description holds for an arbitrary equation of state. Beyond the weak friction approximation it was formally derived for a homogeneous incompressible body [33].
} 
Note that the matrix $\kappa_{L}^{L^{\prime}}$ is odd w.r.t. $L \leftrightarrow L^{\prime}$. We see that in the case of a spinning body the dissipative response contribution may not vanish even if the external perturbation is purely static in a non-rotating frame, i.e. $\partial_{t} \mathcal{E}_{L}=0$. Physically, this can be interpreted as a result of frame-dragging, i.e. the fact that the static sources are viewed as time-dependent by locally rotating observers [35]. ${ }^{4}$ In this case, eq. (2.6) takes the following form

$$
I_{L}=\left(\lambda_{\ell} \delta_{L L^{\prime}}-\nu_{\ell}\left(\delta_{L L^{\prime}} \partial_{t}+\kappa_{L L^{\prime}}\right)\right) \mathcal{E}_{L^{\prime}}, \quad \text { where } \quad \kappa_{L L^{\prime}}=-\kappa_{L^{\prime} L}
$$

We see that rotating bodies can generate an antisymmetric tensorial response even for static external perturbations. Switching to spherical harmonics and using the frequency-space ansatz $\mathcal{E}_{L} \propto e^{-i \omega t}$, we can recast eq. (2.9) in the form similar to eq. $(2.7)^{5}$

$$
I_{\ell m}=\left(\lambda_{\ell}+i \nu_{\ell}(\omega-m \Omega)\right) \mathcal{E}_{\ell m},
$$

where $\Omega$ is body's angular velocity and $m$ is the azimuthal ("magnetic") harmonic number. Note the appearance of the term $\omega-\Omega m$ is generic for rotating bodies [37], it is reminiscent of superradiant scattering [38]. Also note that this term is clearly of non-conservative origin because it is odd under the time reversal transformation $\omega \rightarrow-\omega, m \rightarrow-m$. If body's viscosity is not negligible, the dissipative contribution can survive even if the external tidal environment is static, i.e. $\omega=0$. It is well known that $\nu_{\ell} \neq 0$ for both static and spinning black holes, which manifests itself e.g. in graviton absorption and superradiance $[4,5,38$ 40]. Hence, non-vanishing of the dissipative response in eq. (2.10) for spinning black holes is to be expected.

The upshot of our discussion is that a rigidly rotating spherically-symmetric body develops an antisymmetric tensorial response to weak static external perturbations. These responses correspond to the imaginary part of harmonic-space response coefficients. We will see that a similar picture also holds in a more general case when the body's equilibrium configuration is not spherically-symmetric. In this situation it is convenient to use the following general ansatz for the static response in a non-rotating frame [25],

$$
I_{L}=\lambda_{L L^{\prime}} \mathcal{E}_{L^{\prime}}, \quad I_{\ell m}=\lambda_{\ell m} \mathcal{E}_{\ell m}
$$

Using the point-particle EFT [27], it can be shown that the part of $\lambda_{L L^{\prime}}$ which is even under $L \leftrightarrow L^{\prime}$ corresponds to conservative tidal deformations, whereas the antisymmetric part of $\lambda_{L L^{\prime}}$ captures non-conservative effects such as tidal dissipation. This will be discussed in detail in section 3. Using the isomorphism between the STF tensors and spherical harmonics, one can also separate conservative and dissipative responses at the level of relevant harmonic coefficients $I_{\ell m}$. In this case dissipative effects are encoded in imaginary parts of harmonic-space tidal response coefficients, which map onto the antisymmetric w.r.t. $L \leftrightarrow L^{\prime}$ response tensors, whereas the real part of harmonic coefficients captures the tidal

\footnotetext{
${ }^{4}$ See also ref. [36], which shows that a slowly rotating body affected by a stationary external field produces a dynamical response.

${ }^{5}$ To get this one has to use that $\Omega_{i j}=\Omega \varepsilon_{i k j} \hat{z}_{k},\left(\varepsilon_{i j k}\right.$ is the Levi-Civita symbol, $\left.\hat{z}_{k}=\delta_{k}^{3}\right)$, then contract $\kappa_{L}^{L^{\prime}} \mathcal{E}_{L^{\prime}}$ with $n^{\langle L\rangle}$, expand $n^{\langle L\rangle}$ and $\mathcal{E}_{L^{\prime}}$ over the STF tensor basis and use identities (A.16) from appendix A.
} 
Love numbers and maps onto the response tensors that are even w.r.t. $L \leftrightarrow L^{\prime}$. This suggests that it is more appropriate to call quantities $\lambda_{\ell m}$ defined in eq. (2.11) "tidal response coefficients", and reserve the term "Love numbers" only for its conservative real part.

All in all, the aggregate potential produced by an external static perturbation (2.4) takes the following form ${ }^{6}$

$$
U_{\text {pert }}=-\frac{(\ell-2) !}{\ell !} \sum_{\ell=2} \sum_{m=-\ell}^{\ell} Y_{\ell m} \mathcal{E}_{\ell m} r^{\ell}\left[1+k_{\ell m}\left(\frac{r}{r_{s}}\right)^{-2 \ell-1}\right],
$$

where "pert" means that we have subtracted the body's internal multipole moments, ${ }^{7}$ $r_{s}$ is body's equilibrium radius (in our context this will be black hole's Schwarzschild radius $\left.r_{s}=2 M\right)^{8}$ and $k_{\ell m}$ are dimensionless tidal response coefficients in the Newtonian approximation, defined as

$$
\lambda_{\ell m}=-k_{\ell m} \frac{(\ell-2) !}{(2 \ell-1) ! !} r_{s}^{2 \ell+1} .
$$

Relativistic picture. So far our discussion has been entirely in the realm of the Newtonian approximation, which is only a long-distance approximation to the full general relativity picture. In this more general case, it is convenient to look at the temporal metric component $g_{00}=-1+2 h_{00}$ in body's local asymptotic rest-frame [34, 42], which generalizes the Newtonian potential in general relativity. In the case of perturbations around the Schwarzschild and Kerr black hole solutions, it can be written as [10, 20, 25, 43] ${ }^{9}$

$$
\begin{aligned}
h_{00}^{\text {pert }}=-\frac{(\ell-2) !}{\ell !} \sum_{\ell=2} \sum_{m=-\ell}^{\ell} Y_{\ell m} \mathcal{E}_{\ell m} r^{\ell}[ & \left(1+a_{1} \frac{r_{s}}{r}+\ldots\right) \\
& \left.+k_{\ell m}\left(\frac{r}{r_{s}}\right)^{-2 \ell-1}\left(1+b_{1} \frac{r_{s}}{r}+\ldots\right)\right],
\end{aligned}
$$

where $a_{1}$ and $b_{1}$ are some calculable spin-dependent coefficients whose exact expressions are omitted for clarity. This expression asymptotes eq. (2.12) for the Newtonian potential in the long-distance limit and hence, at first glance, provides us with a practical prescription to extract the tidal response coefficients from a given gravitational potential produced by an external source in full general relativity. Indeed, apparently, we only need to Taylor expand this potential at spatial infinity and read off the coefficient in front of the $r^{-\ell-1}$ power in the expansion. In what follows we will denote this procedure by Newtonian matching. It is important to stress that the response coefficients that we have discussed so far, and which can be extracted by means of the Newtonian matching, are referred to as the electric type Love numbers [10]. There also exist so-called magnetic-type response

\footnotetext{
${ }^{6}$ Note that our response coefficients differ from those of refs. [10, 25] by a factor of 2 .

${ }^{7}$ For black holes these multipole moments can be straightforwardly extracted from the Kerr metric, see e.g. [41].

${ }^{8}$ This choice is only a matter of convention. We could have equally chosen this scale to be, e.g. the difference between the outer and inner horizons of Kerr black holes $r_{+}-r_{-}$. This would only lead to a trivial rescaling of response coefficients by a constant factor.

${ }^{9}$ The fact that the gravitational potential of the Kerr metric reduces to the flat space expression (2.12) follows from the fact that the Kerr spacetime is asymptotically flat.
} 
coefficients, which do not have counterparts in the Newtonian approximation [10, 20]. We will discuss them in section 5 .

Newtonian matching for electric response coefficients was justified in refs. [10, 11], which showed that it gives a gauge-independent result for the Schwarzschild background in four dimensions. However, from the expression (2.14) we see that in the physical case $\ell \in \mathbb{N} /\{1\}$ there may be some ambiguity if the subleading corrections to the source appear to have the same power exponent as the response contribution, i.e. the source and response series overlap. We will see shortly that this ambiguity indeed takes place in the case of the Kerr background when the perturbations are studied in the advanced Kerr coordinates. A similar ambiguity takes place for Love numbers of Schwarzschild black holes in certain higher dimensions. To avoid this ambiguity, Kol and Smolkin [11] have suggested to use the analytic continuation into higher dimensions, where generically the overlap between the source and response series does not happen [11]. Recently Le Tiec et al. (2020) [25] have applied a similar analytic continuation in four dimensions. To that end, it is enough to treat $\ell$ as a generic rational number $\ell \rightarrow \mathbb{R}$. In our work, we show that this approach indeed allows one to avoid the ambiguity and moreover, is motivated from the EFT point of view. We will also show that the EFT itself provides us with an unambiguous way to define the conservative part of the tidal response, i.e. the actual Love numbers.

Finally, a comment on the role of the no-hair theorems [44-47] is in order. In our context these theorems essentially state that external perturbing fields cannot smoothly generate a non-vanishing static profile on top of the Kerr metric. This means that when the external source is adiabatically turned on, the generalized gravitational potential in eq. (2.14) should be uniquely defined by the source itself and body's response. In other words, the no-hair theorems guarantee that black holes' response is analytic in the vicinity of $\omega=0$, there is no gravitational hysteresis (apart from a possible change of black hole's mass and spin) - once the source is turned off, the relevant solution will tend to the Kerr metric again. Thus, it is only because of the no-hair theorems that the decomposition (2.14) is unique and the definition of the response coefficients for black holes is meaningful.

\section{Scalar response coefficients}

In this section we will compute the response of a Kerr black hole to an external spin-0 perturbation. In analogy with the tidal response, it will be referred to as a scalar response coefficient (SRC). First, we will discuss the definition of SRCs in the Newtonian approximation and in the point-particle EFT. Then we will compute the SRCs by solving the scalar field equation of motion (i.e. the spin-0 Teukolsky equation [48-51]) in the Kerr background. We will do so in two different coordinate systems and show that the results agree only if we analytically continue the orbital number of relevant scalar perturbations to non-integer values. We also justify this procedure from the EFT point of view. Finally, we present an explicit matching between the EFT and general relativity calculations. We will show that the dissipative response is captured by complex tidal response coefficients, whereas their real part describes conservative deformations that are natural to associate with classic Love numbers. The scalar response coefficients happen to be purely imaginary, and hence we can conclude that the scalar Love numbers are identically zero for Kerr black holes. 


\subsection{Definition from Newtonian matching}

The response coefficients for a free scalar field can be defined in analogy with the Newtonian gravitation potential outside a generic static body tidally deformed by a weak external gravitational field. Indeed, the fully relativistic Klein-Gordon equation for a static massless scalar field $\Phi$ reduces to the Poisson equation on very large scales. Therefore, in the asymptotic limit $r \rightarrow \infty$ the static scalar field takes the standard expression of the Newtonian potential, which can be split into contributions from an external source and body's response similarly to eq. (2.12),

$$
\left.\Phi\right|_{r \rightarrow \infty}=\sum_{\ell=1} \sum_{m=-\ell}^{\ell} Y_{\ell m} \mathcal{E}_{\ell m}^{(0)} r^{\ell}\left[1+k_{\ell m}^{(0)}\left(\frac{r}{r_{s}}\right)^{-2 \ell-1}\right] .
$$

Note that unlike the gravitation potential, whose multipole expansion can only start with $\ell=2$ by virtue of the equivalence principle, a generic scalar field is allowed to have a non-trivial dipole moment. For this reason we have omitted the normalization factors used in eq. (2.12).

It is known that the full solution to the static Klein-Gordon equation in the Schwarzschild and Kerr backgrounds factorizes in the spherical coordinates, and hence it can be written in the following form $[11,52,53]$

$$
\Phi=\sum_{\ell=1} \sum_{m=-\ell}^{\ell} Y_{\ell m} \mathcal{E}_{\ell m}^{(0)} r^{\ell}\left[\left(1+a_{1} \frac{r_{s}}{r}+\ldots\right)+k_{\ell m}^{(0)}\left(\frac{r}{r_{s}}\right)^{-2 \ell-1}\left(1+b_{1} \frac{r_{s}}{r}+\ldots\right)\right]
$$

where $a_{1}, b_{1}$ are some calculable constants, which depend on black hole's spin. At face value, this gives us a tool to extract the SRCs from the full GR solution: we just have to Taylor expand this solution in $r_{s} / r$ and read off the coefficient in front of the $r^{-\ell-1}$ term. However, at this point it is not clear if the Newtonian definition of SLNs is unambiguous. This disadvantage can be avoided if the Love numbers are defined within the point-particle effective field theory.

\subsection{Static response in the EFT}

In what follows we will use the point particle effective field theory that includes our test scalar field $\Phi$, along with the long-wavelenght metric field $g_{\mu \nu}^{L}$, and the position of the compact object $x^{\mu}$ (see $\left.[4,5,7]\right)$ for further details). Our goal here is to focus on three particular aspects: the EFT definition of Love numbers, a possible ambiguity between the source and response contributions that may appear during the matching of the EFT and microscopic (GR) calculations, and the EFT extension in the presence of spin.

The core idea of the point particle EFT is that any object acts like a point particle when viewed from large enough scales. As we get closer to that object, or as measurement precision becomes better, corrections to the point particle description become important. These finite size effects are captured by higher derivative operators in the context of EFT.

We will start with the non-spinning case, which is sufficient for our purpose to define the Love numbers in a way free of the arbitrariness produced by the response/source split. 
We will re-introduce the Planck mass $M_{P}$ in order to keep track of mass dimensions of the EFT operators. Let us write the following action for a static scalar field coupled to gravity

$$
S=S_{\Phi}^{(2)}+S_{h}^{(2)}+S_{\Phi h}+S_{\mathrm{pp}}+S_{\text {finite-size }}
$$

where $S_{\Phi}^{(2)}$ and $S_{h}^{(2)}$ are the bulk quadratic terms for the scalar field and gravitation, $S_{\Phi h}$ describes the leading interaction between them, $S_{\mathrm{pp}}$ is the point particle worldline action, and the part $S_{\text {finite-size }}$ captures finite-size effects. Let us describe each term separately.

Bulk action. The kinetic term for the bulk scalar field in flat space is given by

$$
S_{\Phi}^{(2)}=-\frac{1}{2} \int d^{4} x \quad \partial_{\mu} \Phi \partial^{\mu} \Phi=-\frac{1}{2} \int d^{4} x \quad\left(\partial_{i} \Phi\right)^{2}
$$

where in the last equality we took the static limit. Now let us focus on the gravitational sector. It is described by expanding the Einstein-Hilbert action in graviton perturbations around the flat background (see e.g. [54]),

$$
g_{\mu \nu}=\eta_{\mu \nu}+2 h_{\mu \nu} .
$$

For the purposes of this section it will be enough to work in the gauge that reproduces the Schwarzschild solution from GR. To that end, we consider the following isotropic perturbations

$$
g_{00}=-\left(1+2 H_{0}(t, r)\right) \quad g_{r r}=\left(1+2 H_{2}(t, r)\right),
$$

with all other components given by the unperturbed Minkowski metric. The kinetic term can be extracted directly from the Einstein-Hilbert action

$$
S_{h}^{(2)}=\frac{M_{P}^{2}}{2} \int d^{4} x \sqrt{-g} R=\frac{M_{P}^{2}}{2} \int d t d \phi d \cos \theta d r\left(2 H_{2}^{2}-4 r\left(\partial_{r} H_{0}\right) H_{2}\right) .
$$

The leading interaction term between the gravitons and the scalar field stems from the scalar field kinetic term,

$$
S_{\Phi h}=\frac{1}{2} \int d t d \phi d \cos \theta d r r^{2}\left(H_{2}-H_{0}\right)\left(\partial_{r} \Phi\right)^{2} \subset-\frac{1}{2} \int d^{4} x \sqrt{-g} g^{\mu \nu} \partial_{\mu} \Phi \partial_{\nu} \Phi .
$$

Point particle action. Finally, we include the worldline action for the black hole. It starts with the point particle part

$$
\begin{aligned}
S_{\mathrm{pp}} & \equiv-M \int d s=-M \int d \tau\left(g_{\mu \nu} \dot{x}^{\mu} \dot{x}^{\nu}\right)^{1 / 2} \\
& =-M \int d^{4} x \int d \tau\left(1+H_{0}\right) \delta^{(4)}(x-x(\tau)),
\end{aligned}
$$

where $M$ is the black hole mass, $d s$ is the infinitesimal proper worldline interval, and $\tau$ is the worldline parameter (proper time); the overdot denotes $d / d \tau$. 
Finite-size effects. As far as the finite size effects are concerned, it is instructive to recall some details of linear response theory $[4,5,27,39,40]$. The worldline action describing the coupling of a source multipole $I_{L}$ and the tidal field $\mathcal{E}_{L}(x)$ (which can be either the gravitational tidal field or its scalar field analog $\left.\mathcal{E}_{L} \propto \partial_{\langle L\rangle} \Phi\right)$ is given by [5]

$$
S_{I \mathcal{E}}=\frac{1}{2} \int d \tau \int d^{4} x \delta^{(4)}(x-x(\tau)) I_{L}(\tau) \mathcal{E}^{L}(x) .
$$

The tidal field $\mathcal{E}_{L}$ acts like a source for $I_{L}$. Hence, in the linear approximation we can write $^{10}$

$$
\left\langle I_{L}(\tau)\right\rangle=\int d \tau^{\prime} G^{\mathrm{ret}} \cdot L_{L}^{\prime}\left(\tau, \tau^{\prime}\right) \mathcal{E}_{L^{\prime}}\left(x\left(\tau^{\prime}\right)\right)
$$

where $\langle\ldots\rangle$ denotes ensemble-averaging w.r.t. internal degrees of freedom and short-scale modes, and we have introduced the retarded Green's function as follows:

$$
G_{L}^{\mathrm{ret} L^{\prime}}\left(\tau, \tau^{\prime}\right)=-i\left\langle\left[I^{L}(\tau), I^{L^{\prime}}\left(\tau^{\prime}\right)\right]\right\rangle \theta\left(\tau-\tau^{\prime}\right),
$$

where $\theta(x)$ is the Heaviside theta-function. Now we switch to frequency space and use that causal Green's function are analytic around $\omega=0$. Then, the spherical symmetry of the problem dictates the following general expression for the causal Green's function

$$
G_{L}^{\mathrm{ret} L^{\prime}}(\omega)=\sum_{n=0} \omega^{2 n}\left(\hat{\lambda}_{2 n L}^{\text {loc. } L^{\prime}}+i \hat{\lambda}_{2 n+1}^{\text {non-loc. } L_{L}^{\prime}} \omega\right)
$$

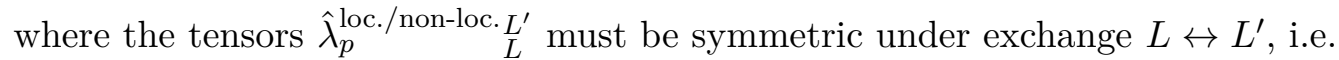

$$
\hat{\lambda}_{p}^{\text {loc./non-loc. } L^{\prime}}=\text { const } \cdot \delta_{\left\langle L^{\prime}\right\rangle}^{\langle L\rangle} \text {. }
$$

The terms in the expansion (3.12) that are symmetric under time-reversal symmetry (i.e., are even under $\omega \rightarrow-\omega$ ) are dubbed local ("loc."). We will see shortly that they correspond to local terms in the effective action for small-wavelength fluctuations. These terms are manifestly time reversal invariant, and hence they correspond to conservative dynamics. However, the terms dubbed non-local ("non-loc.") are not time reversal invariant and hence they describe dissipative effects. Importantly, in the case of the Schwarzschild metric they disappear in the limit of static perturbations $\omega \rightarrow 0$.

The physical response also receives contributions from local operators in the worldline action. Effectively, this leads to a renormalization of the conservative response coefficients. All in all, the total conservative response can be described by a set of the following local wordline operators involving only the long-wavelenght degrees of freedom,

$$
S_{I \mathcal{E}}^{\mathrm{eff}}=\frac{1}{2 \ell !} \int d \tau d^{4} x \delta^{(4)}(x-x(\tau))\left(\lambda_{\ell} \mathcal{E}_{L} \mathcal{E}^{L}+\lambda_{\ell\left(\omega^{2}\right)} \dot{\mathcal{E}}_{L} \dot{\mathcal{E}}^{L}+\ldots\right)
$$

This action can be rewritten in the covariant form by using covariant derivatives along body's 4 -velocity $v^{\mu}=\frac{d x^{\mu}}{d \tau}$ and the projector onto directions orthogonal to $v^{\mu}$

$$
P_{\nu}^{\mu}=\delta_{\nu}^{\mu}+v^{\mu} v_{\nu}, \quad D \equiv v^{\mu} \nabla_{\mu} .
$$

\footnotetext{
${ }^{10}$ For clarity, we have omitted the background multipole moments. These are absent for Schwarschild black holes, but are present for Kerr black holes. These moments can be easily taken into account (see e.g. [40]), but they do not contribute to the tidal response and hence are irrelevant for our discussion.
} 
As a result, the leading worldline interaction term describing finite-size effects in the static limit is given by $[5,7]$

$$
S_{\text {Love }}=\frac{\lambda_{\ell}}{2 \ell !} \int d^{4} x \int d \tau \delta^{(4)}(x-x(\tau))\left[P_{\left\langle\mu_{1}\right.}^{\nu_{1}} \ldots P_{\left.\mu_{\ell}\right\rangle}^{\nu_{\ell}} \partial_{\nu_{1} \ldots \nu_{\ell}} \Phi\right]\left[P_{\sigma_{1}}^{\left\langle\mu_{1}\right.} \ldots P_{\sigma_{\ell}}^{\left.\mu_{\ell}\right\rangle} \partial^{\sigma_{1} \ldots \sigma_{\ell}} \Phi\right]
$$

where we have introduced the multi-derivative operator $\partial_{\nu_{1} \ldots \nu_{\ell}} \equiv \prod_{i=1}^{\ell} \partial_{\nu_{i}}$, whereas $\langle\ldots\rangle$ stands for the symmetrized traceless component. The leading non-static effects are captured by the following action

$$
S_{\omega^{2}}=\left.\frac{\lambda_{\ell\left(\omega^{2}\right)}}{2 \ell !} \int d \tau D\left[P_{\left\langle\mu_{1}\right.}^{\nu_{1}} \ldots P_{\left.\mu_{\ell}\right\rangle}^{\nu_{\ell}} \partial_{\nu_{1} \ldots \nu \ell} \Phi\right] D\left[P_{\sigma_{1}}^{\left\langle\mu_{1}\right.} \ldots P_{\sigma_{\ell}}^{\left.\mu_{\ell}\right\rangle} \partial^{\sigma_{1} \ldots \sigma_{\ell}} \Phi\right]\right|_{x=x(\tau)}
$$

The coupling $\lambda_{\ell}$ will be referred to as the EFT Love number in what follows. Eq. (3.16) can be viewed as a gauge-independent definition of the Love numbers, as the corresponding worldline operator is manifestly covariant. We will see momentarily that it is precisely this operator that generates the $r^{-\ell-1}$ term in the Newtonian expansion. Importantly, we will see that the coupling $\lambda_{\ell}$ does not exhibit logarithmic running in general relativity in four dimensions. This will guarantee that result of the Newtonian matching is meaningful. The situation is different for frequency-dependent Love numbers, which generically depend on distance, and hence introduce some ambiguity in the direct application of the Newtonian matching. We will discuss this in section 6 .

In what follows we will work in the body's rest frame where $v^{\mu}=\delta_{0}^{\mu}$, hence $P_{0}^{\mu}=0$, $P_{j}^{i}=\delta_{j}^{i}$, which removes all operators with time derivatives in eq. (3.16). This gives us the following action relevant for the study of local static response

$$
S_{\text {Love }}=\frac{\lambda_{\ell}}{2 \ell !} \int d^{4} x \int d \tau \delta^{(4)}(x-x(\tau)) \partial_{\left\langle i_{1} \ldots i_{\ell}\right\rangle} \Phi \partial^{\left\langle i_{1} \ldots i_{\ell}\right\rangle} \Phi .
$$

Static response in the EFT. Our goal here is to compute the static scalar field profile $\Phi$ in the presence of interactions with gravitons and an external source $\bar{\Phi}$. As a first step, we need to compute the leading order graviton field, which describes the gravitational potential of the black hole on large scales. In the EFT context, the black hole solution is recovered perturbatively order by order in the long-distance expansion controlled by $2 M /\left(M_{P}^{2} r\right)$. Let us start with the first order. The equations of motion for the graviton modes are given by

$$
H_{2}=r \partial_{r} H_{0}, \quad \Delta H_{0}=\frac{M}{2 M_{P}^{2}} \int d \tau \delta^{(4)}(x-x(\tau)) .
$$

Let us work in the black hole's rest frame, where the unperturbed center-of-mass position is given by $x^{i}(\tau)=0, x^{0}=\tau$, such that

$$
\Delta H_{0}=\frac{M}{2 M_{P}^{2}} \delta^{(3)}(\mathbf{x}) \quad \Rightarrow \quad H_{0}=-\frac{M}{8 \pi M_{P}^{2} r}=-\frac{r_{s}}{2 r},
$$

where we have introduced the Schwarzschild radius $r_{s}=M /\left(4 \pi M_{P}^{2}\right)$. The second metric perturbation is given by

$$
H_{2}=-H_{0}=\frac{r_{s}}{2 r}
$$


Now we have to compute the scalar field profile. The total static equation of motion for the scalar field, which includes the leading interaction with gravity and the tidal response is given by

$$
\Delta \Phi-\frac{2}{r^{2}} \partial_{r}\left(r^{2} H_{2} \partial_{r}\right) \Phi+(-1)^{\ell} \frac{\lambda_{\ell}}{\ell !} \partial_{\left\langle i_{1} \ldots i_{\ell}\right\rangle}\left(\partial^{\left\langle i_{1} \ldots i_{\ell}\right\rangle} \Phi \delta^{(3)}(\mathbf{x})\right)=0 .
$$

In order to compute the black hole response, we introduce an external scalar field source $\bar{\Phi}$, satisfying the free Poisson equation $\Delta \bar{\Phi}=0$ in the $r \rightarrow \infty$ asymptotic. Assuming that $\bar{\Phi}$ has an orbital number $\ell$, we find

$$
\Delta \bar{\Phi}=0 \quad \Rightarrow \quad \bar{\Phi}=\mathcal{E}_{i_{1} \ldots i_{\ell}}^{(0)} x^{i_{1}} \ldots x^{i_{\ell}}
$$

where $\mathcal{E}_{i_{1} \ldots i_{\ell}}^{(0)}$ is a symmetric trace-free tensor. Note that the solution (3.23) corresponds to $\bar{\Phi}=\sum_{\ell m} \mathcal{E}_{\ell m}^{(0)} r^{\ell} Y_{\ell m}(\theta, \phi)$ in the spherical coordinates. We want to solve eq. (3.22) perturbatively expanding in $r_{s} / r$, but keep the explicit $\lambda_{\ell}$-dependence,

$$
\Phi=\bar{\Phi}+\Phi_{h}^{(1)}+\Phi_{\text {Love }}^{(1)}+\ldots
$$

We will formally retain corrections linear in $\lambda_{\ell}$, but $\lambda_{\ell}$ itself does not need to be small. Let us first compute the correction to the source coming from the interaction with the graviton. We have

$$
\Delta \Phi_{h}^{(1)}=\frac{1}{r^{2}} \partial_{r}\left(r^{2} 2 H_{2} \partial_{r}\right) \bar{\Phi} .
$$

Expanding $\Phi_{h}^{(1)}$ over spherical harmonics and using the solution from eq. (3.21) we obtain

$$
\left(\partial_{r}^{2}+\frac{2}{r} \partial_{r}-\frac{\ell(\ell+1)}{r^{2}}\right) \Phi_{h \ell m}^{(1)}=\frac{r_{s}}{r^{2}} \partial_{r}\left(r \partial_{r} \bar{\Phi}\right) .
$$

Note that in the above expression we can lift all restrictions on $\ell$ and treat it as a generic number. Plugging our source from eq. (3.23), this equation can be easily solved. The full solution including the source plus the leading graviton correction is given by

$$
\Phi=\sum_{m=-\ell}^{\ell} \mathcal{E}_{\ell m}^{(0)} Y_{\ell m} r^{\ell}\left(1-\frac{\ell}{2} \frac{r_{s}}{r}\right)=\mathcal{E}_{i_{1} \ldots i_{\ell}}^{(0)} x^{i_{1}} \ldots x^{i_{\ell}}\left(1-\frac{\ell}{2} \frac{r_{s}}{r}\right) .
$$

We observe that the interaction with the graviton induces the sub-leading corrections to the source. Note that this correction is calculable, i.e. its strength is fixed in the EFT itself. Importantly, because the graviton propagator scales like $1 / r$, these corrections are naturally organized as the following power series

$$
\Phi \supset r^{\ell}\left(1+c_{1} \frac{r_{s}}{r}+c_{2}\left(\frac{r_{s}}{r}\right)^{2}+\ldots\right),
$$

for any $\ell \in \mathbb{R}$. This gives an interpretation of the subleading source corrections: these are just generated by the coupling between the source and perturbative gravity. Our result also justifies the use of the analytic continuation $\ell \rightarrow \mathbb{R}$ for the source-response split, because 
this indeed allows one to isolate the series eq. (3.28) and avoid a possible overlap with corrections induced by finite-size effects.

Now let us compute the correction to the source coming from the Love interaction, which corresponds to induced multipoles. We have

$$
\Delta \Phi_{\text {Love }}^{(1)}=-(-1)^{\ell} \lambda_{\ell} \mathcal{E}^{(0)^{i_{1} \ldots i_{\ell}}} \partial_{i_{1} \ldots i_{\ell}} \delta^{(3)}(\mathbf{x}) .
$$

This equation can be easily solved in Fourier space,

$$
\begin{aligned}
\Phi_{\text {Love }}^{(1)} & =(-i)^{\ell} \lambda_{\ell} \mathcal{E}^{(0)}{ }_{i_{1} \ldots i_{\ell}} \int \frac{d^{3} k}{(2 \pi)^{3}} e^{i \mathbf{k} \cdot \mathbf{x}} \frac{k_{i_{1}} \ldots k_{i_{\ell}}}{\mathbf{k}^{2}} \\
& =B_{\ell} \lambda_{\ell} \mathcal{E}^{(0)}{ }_{i_{1} \ldots i_{\ell}} x^{i_{1}} \ldots x^{i_{\ell}} \frac{1}{r^{2 \ell+1}},
\end{aligned}
$$

where we have introduced the following normalization constant

$$
B_{\ell} \equiv(-1)^{\ell} \frac{2^{\ell-2}}{\pi^{1 / 2} \Gamma(1 / 2-\ell)} .
$$

The total solution including linear order correction in $\lambda_{\ell}$ and $r_{s} / r$ is given by

$$
\Phi=\mathcal{E}_{{ }_{1} \ldots i_{\ell}}^{(0)} x^{i_{1}} \ldots x^{i_{\ell}}(\underbrace{1}_{\text {source }}-\underbrace{\frac{\ell}{2} \frac{r_{s}}{r}}_{\text {graviton interaction }}+\underbrace{B_{\ell} \lambda_{\ell} \frac{1}{r^{2 \ell+1}}}_{\text {induced multipole }}) .
$$

Comparing this to (3.2), we can see that the EFT provides a tool to define the response coefficients and avoid ambiguity in the source/response split. The subleading corrections in the source expansion, which scale as $\left(r_{s} / r\right)^{n} \times r^{\ell}$, naturally correspond to diagrams produced by the interaction between the source field and the graviton. These diagrams are fixed by the structure of Einstein-Hilbert and the Klein-Gordon actions. Thus, all graviton corrections can be unambiguously computed by expanding the Einstein-Hilbert equation in higher order operators involving the graviton field. Hence, in principle, during the matching procedure, one can identify all corrections coming from the graviton vertices and subtract them from the microscopic solution obtained in GR. The remaining piece will correspond to the response coefficients. In practice, however, the number of diagrams to be computed can be very large. From the practical point of view, it is more convenient to do the analytic continuation $\ell \rightarrow \mathbb{R}$, which allows one to easily achieve the same goal of isolating non-linear corrections to the source from those generated by the finite-size effects.

Inclusion of spin. In order to reproduce the GR solution in the EFT, one has to perturbatively recover the Kerr metric at order $a^{2} / r^{2}$, where $a \equiv J / M$ is the normalized black hole's spin. To that end one has to introduce vector degrees of freedom of metric perturbations and consider their coupling to black hole's spin via the MathissonPapapetrou/Routhian formalism [5]. This procedure has been recently presented in ref. [27], see also refs. $[55,56]$. In order to obtain the $a^{2} / r^{2}$ corrections, we need to take into account the cubic interaction between the scalar and vector graviton modes. The details of 
this calculation are not essential for our discussion. Once we obtain the following graviton perturbation $^{11}$

$$
h_{\phi \phi}=\frac{a^{2}}{r^{2}} \cdot r^{2} \sin ^{2} \theta
$$

it can be coupled to the scalar field through the kinetic term,

$$
\int d^{4} x \sqrt{-g} g^{\mu \nu} \partial_{\mu} \Phi \partial_{\nu} \Phi \supset \int d^{4} x h^{\phi \phi}\left(\partial_{\phi} \Phi\right)^{2} \propto \int d t d \phi d \theta d r r^{2} \sin \theta\left[\frac{a^{2} m^{2}}{r^{4}} \Phi^{2}\right],
$$

where in the last equation we used the expansion over spherical harmonics $\Phi \propto e^{i m \phi}$. Then, we can easily account for perturbations that are produced by the interactions between the source and the gravitons at the leading order in spin. Varying the action (3.34) over $\Phi$ and using the perturbative expansion

$$
\Phi=\bar{\Phi}+\Phi_{h}^{(1)}+\Phi_{a}^{(1)}
$$

we obtain that the correction due to black hole spin $\Phi_{a}^{(1)}$ satisfies the following differental equation

$$
\left(\partial_{r}^{2}+\frac{2}{r} \partial_{r}-\frac{\ell(\ell+1)}{r^{2}}\right) \Phi_{a}^{(1)}=\frac{a^{2}}{r^{4}} \partial_{\phi}^{2} \bar{\Phi} .
$$

Using our ansatz for the source (3.23), we obtain the following net expression for the source interacting with the Kerr black hole at the leading orders in black hole's mass and spin

$$
\Phi=\sum_{m} \mathcal{E}^{(0)}{ }_{\ell m} Y_{\ell m} r^{\ell}\left(1-\frac{\ell}{2} \frac{2 M}{r}+\frac{m^{2} a^{2}}{4 \ell-2} \frac{1}{r^{2}}\right) .
$$

Iterating this procedure at higher orders in $r_{s} / r$, we can reconstruct all power series responsible for the interaction between the source and the graviton.

Finite size operators in the presence of spin. The structure of finite-size operators becomes more complicated due to the spin, because now we can produce new tensor structures using the spin vector $s^{i}$ and the Levi-Civita antisymmetric symbol $\epsilon_{i j k}$. Therefore, now we can write the following general expression [27] for the causal response function introduced in eq. (3.10)

$$
G_{L}^{\text {ret } L^{\prime}}(\omega)=\sum_{n=0} \omega^{2 n}\left(\hat{\lambda}_{2 n}^{\text {loc. } L^{\prime}}+i \hat{\epsilon}_{2 n+1}^{\text {loc. }} L_{L^{\prime}} \omega+\hat{\epsilon}_{2 n}^{\text {non-loc. } L_{L}^{\prime}}+i \hat{\lambda}_{2 n+1}^{\text {non-loc. } L} L_{L^{\prime}} \omega\right)
$$

Let us now specify symmetry properties of different response tensors in this expression. From the axial symmetry of the problem, the tensors that are even and odd w.r.t. $L \leftrightarrow L^{\prime}$ must be constructed from even and odd numbers of spin vectors, respectively. ${ }^{12}$ The local terms in eq. (3.38) must be symmetric w.r.t. time reversal invariance (which includes the spin flip), which now corresponds to simultaneous exchange $L \leftrightarrow L^{\prime}, \omega \rightarrow-\omega$. These terms

\footnotetext{
${ }^{11} \mathrm{We}$ assume a gauge consistent with the Boyer-Lindquist coordinates [57].

${ }^{12}$ The only available tensor structures are $\delta_{i j}, \epsilon_{i j k}$ and $s_{j}=s z_{j}$, where $z_{j}=\delta_{j}^{3}$. Hence, any tensor that is odd w.r.t. $L \leftrightarrow L^{\prime}$ (but is still STF w.r.t. multi-indices $L$ and $L^{\prime}$ ) has to look like $\epsilon_{i i^{\prime} k} s_{k}$ times a tensor that is even w.r.t. remaining multi-index exchange $(L-1) \leftrightarrow(L-1)^{\prime}$.
} 
correspond to local operators in the point-particle EFT in the body's rotation frame. In contrast, the non-local terms correspond to internal (gapless) worldline degrees of freedom that capture dissipation [27] and hence they must be odd w.r.t. exchange $L \leftrightarrow L^{\prime}, \omega \rightarrow-\omega$. This implies that tensors $\hat{\lambda} \hat{\lambda}_{p}^{\text {loc./non-loc. } L_{L}^{\prime}}$ and $\hat{\epsilon}_{p}^{\text {loc./non-loc. } L_{L}^{\prime}}$ in eq. (3.38) must be even and odd w.r.t. $L \leftrightarrow L^{\prime}$, respectively. Going back to proper time we get,

$$
\left\langle I_{L}\right\rangle=\sum_{n}(-1)^{n}\left(\hat{\lambda}_{2 n}^{\text {loc. } L^{\prime}}+\hat{\epsilon}_{2 n+1}^{\text {loc. }} \frac{L}{L^{\prime}} \frac{d}{d \tau}+\hat{\epsilon}_{2 n}^{\text {non-loc. } L_{L}^{\prime}}+\hat{\lambda}_{2 n+1}^{\text {non-loc. } L} \frac{d}{L^{\prime}} \frac{d}{d \tau}\right) \frac{d^{2 n}}{d \tau^{2 n}} \mathcal{E}_{L^{\prime}} .
$$

Unlike the Schwazschild black hole, the terms with time derivatives here do not vanish in the static limit (w.r.t. a fixed inertial frame) because of the rotation of the body. Hence, if we want to capture the effects of spin to all orders, we need to keep track of all powers of frequency here. This problem can be solved if we rewrite eq. (3.38) in a fixed inertial frame. To that end we can expand time derivatives in body's rotation frame as

$$
\left.\frac{d}{d \tau} \mathcal{E}_{L^{\prime}}=\partial_{t} \mathcal{E}_{L^{\prime}}-\ell \Omega_{\left(i_{1}^{\prime} q\right.} \mathcal{E}_{q} i_{2}^{\prime} \ldots i_{\ell}^{\prime}\right),
$$

where $\partial_{t}$ is the time derivative in the fixed inertial (source) frame, $\Omega_{i j}$ is the antisymmetric angular velocity tensor, and we have symmetrized the rightmost term w.r.t. its free indices. To simplify the argument, let us neglect terms with partial time derivatives $\partial_{t}$, which is reasonable since we are interested in the static limit.

In this limit, applying an even number of time derivative in the body's local rotating frame will produce a tensor built out of the same even number of the angular velocity tensors. Multiplying an original response matrix by this tensor will not change its parity properties w.r.t. multi-index exchange,

$$
\hat{\lambda}_{2 n L}^{\text {loc. } L^{\prime}} \frac{d^{2 n}}{d \tau^{2 n}} \rightarrow \lambda_{2 n L}^{\text {loc. } L^{\prime}}\left(\text { even } L \leftrightarrow L^{\prime}\right), \quad \hat{\epsilon}_{2 n}^{\text {non-loc. } L^{\prime}} \frac{d^{2 n}}{d \tau^{2 n}} \rightarrow \epsilon_{2 n}^{\text {non-loc. } L^{\prime}}\left(\text { odd } L \leftrightarrow L^{\prime}\right),
$$

where $\lambda_{2 n}$ and $\epsilon_{2 n}$ are response tensors written in the inertial frame. However, applying an odd number of time derivatives produces a tensor that contains the same odd number of the angular velocity tensors. This tensor is odd w.r.t. to the exchange of its multi-indices, and hence it will change the parity of the corresponding response matrices w.r.t. exchange $L \leftrightarrow L^{\prime}$,

$\hat{\lambda}_{2 n+1}^{\text {non-loc. } L_{L}^{\prime}} \frac{d^{2 n+1}}{d \tau^{2 n+1}} \rightarrow \lambda_{2 n}^{\text {non-loc. } L^{\prime}}\left(\right.$ odd $\left.L \leftrightarrow L^{\prime}\right), \quad \hat{\epsilon}_{2 n+1}^{\text {loc. }} \frac{L^{\prime}}{d \tau^{2 n+1}} \rightarrow \epsilon_{2 n+1 L}^{\text {loc. } L^{\prime}}\left(\right.$ even $\left.L \leftrightarrow L^{\prime}\right)$,

where $\lambda_{2 n+1}$ and $\epsilon_{2 n+1}$ are again response tensors in the inertial frame. We see that after we have changed the frame, all terms of non-local origin can be collected into a new antisymmetric response matrix $\kappa_{L}^{L^{\prime}}$, whereas all local terms effectively sum up into a new symmetric matrix $\lambda_{L}^{L^{\prime}}$. Importantly, the transition to the inertial frame did not mix the properties of the response matrices written in eq. (3.38) - the local response is still captured by a matrix that is even w.r.t. $L \leftrightarrow L^{\prime}, \omega \rightarrow-\omega$. All in all, we can write

$$
\begin{aligned}
\left\langle I_{L}\right\rangle & =\left(\lambda_{L}^{L^{\prime}}+\kappa_{L}^{L^{\prime}}\right) \mathcal{E}_{L^{\prime}}+\left(\Lambda_{L}^{L^{\prime}}+\Lambda_{L}^{\prime L^{\prime}}\right) \partial_{t} \mathcal{E}_{L^{\prime}}+\ldots, \quad \text { where } \\
\lambda_{L}^{L^{\prime}} & =\lambda_{L^{\prime}}^{L}, \quad \kappa_{L}^{L^{\prime}}=-\kappa_{L^{\prime}}^{L}, \quad \Lambda_{L}^{L^{\prime}}=-\Lambda_{L}^{L^{\prime}}, \quad \Lambda_{L}^{L^{\prime}}=\Lambda_{L^{\prime}}^{L},
\end{aligned}
$$


and "..." stands for operators that involve more than one partial time derivatives. We stress that the instantaneous contribution proportional to $\kappa_{L^{\prime}}^{L}$, in fact, stems from non-local operators, which was nicely explained in ref. [27]. The corresponding induced multipole $I_{L} \propto \kappa_{L}^{L^{\prime}} \mathcal{E}_{L^{\prime}}$ describes dissipative effects such as tidal torques or mass loss/accretion [25, 27]. In contrast, the part of the response proportional to $\lambda_{L^{\prime}}^{L}$ in eq. (3.43) captures the local static deformation produced by external fields, it is indistinguishable from the effect of local operators in the point particle action.

All in all, the leading-order local finite-size effects are described by the following generalized response operator (3.16),

$$
S_{\text {Love }}^{a}=\frac{1}{2 \ell !} \int d^{4} x \int d \tau \delta^{(4)}(x-x(\tau)) \lambda^{(0)^{j_{1} \ldots j_{\ell}}}{ }_{i_{1} \ldots i_{\ell}}^{j_{\left\langle j_{1} \ldots j_{\ell}\right\rangle}} \Phi \partial^{\left\langle i_{1} \ldots i_{\ell}\right\rangle} \Phi,
$$

which accounts for the violation of the spherical symmetry by the spinning black hole background. The coupling eq. (3.44) can always be recast in the manifestly covariant form by means of the projection operator (3.15). Note that $\lambda$ is a symmetric trace-free tensor w.r.t. upper and lower sets of indices,

$$
\lambda^{(0)}{ }_{i_{1} \ldots i_{\ell}}^{j_{1} \ldots j_{\ell}}=\lambda^{(0)} \underset{\left\langle i_{1} \ldots i_{\ell}\right\rangle}{\left\langle j_{1} \ldots j_{\ell}\right\rangle},
$$

but its trace w.r.t. the contraction of lower and upper indices does not vanish. We will refer to $\lambda^{(0)}$ and its analogs for higher spins as "Love tensor" in what follows.

The calculation of the response of the scalar field induced by this operator is identical to one presented above, and it yields

$$
\Phi_{\text {Love }}^{(1)}=B_{\ell} r^{-\ell-1} \mathcal{E}^{(0)} L^{\prime} n^{L} \lambda_{L}^{(0)}{ }_{L}^{L^{\prime}},
$$

where the constant $B_{\ell}$ is given in eq. (3.31).

\subsection{Microscopic computation}

To find the SRCs $k_{\ell m}^{(0)}$, we need to solve the vacuum Klein-Gordon equation in the Kerr background, assuming that the scalar field varies very slowly in time. It is instructive to carry out our microscopic (i.e. general relativity) calculation in two different coordinate systems. Let us start with the advanced Kerr coordinates, which are manifestly regular at the horizon.

Advanced Kerr coordinates. The interval of the Kerr spacetime in the advanced Kerr coordinates is given by [58]

$$
\begin{aligned}
d s^{2}= & -\left(1-\frac{2 M r}{\Sigma}\right) d v^{2}+2 d v d r-\frac{4 M r a}{\Sigma} \sin ^{2} \theta d v d \tilde{\phi}-2 a \sin ^{2} \theta d r d \tilde{\phi} \\
& +\Sigma d \theta^{2}+\left(r^{2}+a^{2}+\frac{2 M r}{\Sigma} a^{2} \sin ^{2} \theta\right) \sin ^{2} \theta d \tilde{\phi}^{2},
\end{aligned}
$$

where $a \equiv J / M$ is the reduced spin parameter and $\Sigma \equiv r^{2}+a^{2} \cos ^{2} \theta$. It is well known that in the static case $(\omega=0)$ the Klein-Gordon equation for the massless scalar field factorizes 
into usual scalar spherical harmonics in the Kerr background $[52,59]$. In the advanced Kerr coordinates this decomposition takes the following form

$$
\Phi=\sum_{\ell m} \mathcal{E}_{\ell m}^{(0)} \tilde{R}_{\ell m}(r) Y_{\ell m}(\theta, \tilde{\phi})
$$

To match the source boundary condition at infinity, we impose $\tilde{R}_{\ell m} \rightarrow r^{\ell}$ at $r \rightarrow \infty$ and demand this function to be smooth at the external black hole horizon. The equation defining the radial mode function $\tilde{R}_{\ell m}$ takes the following form

$$
x(1+x) \tilde{R}_{\ell m}^{\prime \prime}(x)+[(1+2 x)+2 i m \gamma] \tilde{R}_{\ell m}^{\prime}(x)-\ell(\ell+1) \tilde{R}_{\ell m}(x)=0,
$$

where $^{\prime} \equiv \partial / \partial x$ and we have defined

$$
x \equiv \frac{r-r_{+}}{r_{+}-r_{-}}, \gamma \equiv \frac{a}{r_{+}-r_{-}}, \quad r_{ \pm}=M \pm \sqrt{M^{2}-a^{2}} .
$$

Note that $r_{+}$and $r_{-}$are the outer and inner horizons of the Kerr black hole, respectively. In what follows we will be mostly focusing on $r_{+}$and we will refer to it simply as "black hole's horizon." We will also use the following notation

$$
r_{s a} \equiv r_{+}-r_{-}
$$

The solution of eq. (3.49) regular at the horizon $(x \rightarrow 0)$ is given by

$$
\tilde{R}_{\ell m}=\text { const } \cdot{ }_{2} F_{1}(\ell+1,-\ell ; 1+2 i m \gamma,-x),
$$

where ${ }_{2} F_{1}$ stands for the Gauss hypergeometric function (see appendix A for more detail). In the physical case $\ell \in \mathbb{N}$, the function $\tilde{R}_{\ell m}$ is a polynomial in $x \propto r$ and hence it does not contain any decaying power of $r$. Thus, by looking at eq. (3.2) one may be tempted to conclude that the SRCs are zero for the Kerr background. However, we have to ensure that this is not a result of a cancellation between body's response and the subleading source contributions due to graviton interactions. To that end, let us consider an analytic continuation $\ell \rightarrow \mathbb{R}$, in which case the solution eq. (3.52) can be Taylor-expanded at spatial infinity as (see appendix A)

$$
\begin{aligned}
\tilde{R}_{\ell m}= & \text { const } \cdot\left(\frac{\Gamma(1+2 i m \gamma) \Gamma(2 \ell+1)}{\Gamma(\ell+1) \Gamma(1+\ell+2 i m \gamma)} x^{\ell} \cdot{ }_{2} F_{1}\left(-\ell,-\ell-2 i m \gamma,-2 \ell,-x^{-1}\right)\right. \\
& \left.+\frac{\Gamma(1+2 i m \gamma) \Gamma(-2 \ell-1)}{\Gamma(-\ell) \Gamma(-\ell+2 i m \gamma)} x^{-\ell-1} \cdot{ }_{2} F_{1}\left(\ell+1, \ell+1-2 i m \gamma, 2 \ell+2,-x^{-1}\right)\right) \\
& \underset{x \rightarrow \infty}{\longrightarrow} r_{s a}^{\ell} x^{\ell}\left(1+\frac{\Gamma(-2 \ell-1) \Gamma(\ell+1) \Gamma(1+\ell+2 i m \gamma)}{\Gamma(2 \ell+1) \Gamma(-\ell) \Gamma(-\ell+2 i m \gamma)} x^{-2 \ell-1}\right) .
\end{aligned}
$$

Since the first distinctive contribution in eq. (3.53) scales as $r^{\ell}$ at infinity, it is natural to associate it with the external source and its corrections produced by non-linear gravitational interactions. The second distinctive contribution in eq. (3.53) scales as $r^{-\ell-1}$ at infinity, and hence it is natural to interpret it as black hole's response. Comparing eq. (3.53) with 
the (post-)Newtonian expansion formula (3.2), we find that for a general multipolar index $\ell$ the response coefficients are given by

$$
k_{\ell m}^{(0)}=\frac{\Gamma(-2 \ell-1) \Gamma(\ell+1) \Gamma(1+\ell+2 i m \gamma)}{\Gamma(2 \ell+1) \Gamma(-\ell) \Gamma(-\ell+2 i m \gamma)}\left(\frac{r_{s a}}{r_{s}}\right)^{2 \ell+1} .
$$

The scalar tidal response coefficients extracted by means of the analytic continuation do not vanish even in physical limit $\ell \rightarrow \mathbb{N}$. In this case eq. (3.54) can be simplified

$$
\begin{aligned}
k_{\ell m}^{(0)} & =-i m \gamma \frac{(\ell !)^{2}}{(2 \ell) !(2 \ell+1) !} \prod_{n=1}^{\ell}\left(n^{2}+4 m^{2} \gamma^{2}\right)\left(\frac{r_{s a}}{r_{s}}\right)^{2 \ell+1} \\
& =-\frac{i m \chi}{2} \frac{(\ell !)^{2}}{(2 \ell) !(2 \ell+1) !} \prod_{n=1}^{\ell}\left(n^{2}\left(1-\chi^{2}\right)+m^{2} \chi^{2}\right),
\end{aligned}
$$

where in the last line we have introduced the dimensionless spin $\chi \equiv a / M$ and used eq. (A.18). Note that the expression (3.55) vanishes in the limit $\chi \rightarrow 0$, reproducing the well-established result that the scalar response coefficients of non-spinning black holes are zero $[7,11]$. Importantly, the response coefficients (3.55) are purely imaginary. As discussed before, they correspond to dissipative effects and not to the classic conservative static response coefficients which we will refer to as scalar Love numbers.

All in all, we have obtained that the radial solution (3.52) in the advanced Kerr coordinates is a polynomial without any decaying power of $r$, and, at the same time, the response coefficients are non-zero. The only possibility to reconcile these two facts is that the GR corrections to the source and the induced response happened to exactly cancel one another in the advanced Kerr coordinates in the physical limit $\ell \rightarrow \mathbb{N}$. This is exactly what happens. To see this, we expand the relevant source solution at infinity as follows

$$
\begin{aligned}
& x_{2}^{\ell} F_{1}\left(-\ell,-\ell-2 i m \gamma,-2 \ell,-x^{-1}\right) \\
& \quad=x^{\ell} \sum_{n=0}^{\infty} \frac{\Gamma(-\ell+n) \Gamma(-\ell-2 i m \gamma+n) \Gamma(-2 \ell)}{\Gamma(-\ell) \Gamma(-\ell-2 i m \gamma) \Gamma(-2 \ell+n)}(-1)^{n} \frac{x^{-n}}{n !} .
\end{aligned}
$$

Let's focus on the $n=2 \ell+1$ 'th term in the hypergeometric series above. This term scales like $r^{-\ell-1}$ just like the response contribution. We have

$$
\begin{aligned}
& x^{2 \ell+1}{ }_{2} F_{1}\left(-\ell,-\ell-2 i m \gamma,-2 \ell,-x^{-1}\right) \\
& \quad \supset \frac{\Gamma(-2 \ell-1) \Gamma(\ell+1) \Gamma(1+\ell-2 i m \gamma)}{\Gamma(2 \ell+1) \Gamma(-\ell) \Gamma(-\ell-2 i m \gamma)}=i m \gamma \frac{(\ell !)^{2}}{(2 \ell) !(2 \ell+1) !} \prod_{n=1}^{\ell}\left(n^{2}+4 m^{2} \gamma^{2}\right),
\end{aligned}
$$

where we took the physicacl limit $\ell \rightarrow \mathbb{N}$ in the second line. This exactly equals minus the coefficient in front of the response part in eq. (3.53), hence the subleading source contribution exactly cancels the response in the advanced Kerr coordinates.

The upshot of our discussion is that the naive identification of the response coefficients from the solution to the Klein-Gordon equation may be ambiguous due to uncertainty in the source/response split. This ambiguity can be removed by means of the analytic continuation $\ell \rightarrow \mathbb{R}[25]$. This is explicitly confirmed by a calculation in the Boyer-Lindquist coordinates, to which we proceed now. 
Boyer-Lindquist coordinates. The line element of the Kerr spacetime in the BoyerLindquist coordinates [57] is given by

$$
\begin{aligned}
d s^{2}= & -\left(1-\frac{2 M r}{\Sigma}\right) d t^{2}-\left(\frac{4 M a r \sin ^{2} \theta}{\Sigma}\right) d t d \phi+\frac{\Sigma}{\Delta} d r^{2}+\Sigma d \theta^{2} \\
& +\sin ^{2} \theta\left(r^{2}+a^{2}+\frac{2 M a^{2} r \sin ^{2} \theta}{\Sigma}\right) d \phi^{2},
\end{aligned}
$$

where $\Delta \equiv r^{2}-2 M r+a^{2}, \Sigma \equiv r^{2}+a^{2} \cos ^{2} \theta$. The Boyer-Lindquist and advanced Kerr coordinates are related via

$$
d v=d t+d r\left(1+\frac{2 M r}{\Delta}\right), \quad d \tilde{\phi}=d \phi+d r \frac{a}{\Delta} .
$$

The static scalar field equation factorizes in these coordinates as follows [52]

$$
\Phi=\sum_{\ell m} \mathcal{E}_{\ell m}^{(0)} R_{\ell m}(r) Y_{\ell m}(\theta, \phi)
$$

where the radial mode function $R_{\ell m}$ satisfies the following equation:

$$
x(x+1) R_{\ell m}^{\prime \prime}(x)+(2 x+1) R_{\ell m}^{\prime}(x)+\left(-\left(\ell^{2}+\ell\right)+\frac{\gamma^{2} m^{2}}{x(x+1)}\right) R_{\ell m}=0 .
$$

We are looking for a solution which is smooth at the black hole's horizon and has a singularity at spatial infinity. Identifying this solution in the Boyer-Lindquist coordinates is not evident, as these coordinates are singular at the Kerr horizon. Nevertheless, it can be shown that the regularity at the horizon in the Boyer-Lindquist coordinates corresponds to the following condition, obtained by Press (1972) [53] and Teukolsky (1973) [49]

$$
R_{\ell m}=\text { const } \cdot\left(r-r_{+}\right)^{+i m \gamma} \quad \text { as } \quad r \rightarrow r_{+} .
$$

The constant should be chosen such that $R_{\ell m} / r^{\ell} \rightarrow 1$ at $r \rightarrow \infty$. The solution satisfying these boundary conditions is given by

$$
\begin{aligned}
R_{\ell m} & =\mathrm{const} \cdot\left(\frac{x}{1+x}\right)_{2}^{i m \gamma}{ }_{2} F_{1}(\ell+1,-\ell, 1+2 i m \gamma,-x) \\
& \underset{x \rightarrow \infty}{\longrightarrow} r_{s a}^{\ell} \cdot\left(x^{\ell}+\frac{\Gamma(-2 \ell-1) \Gamma(\ell+1) \Gamma(1+\ell+2 i m \gamma)}{\Gamma(2 \ell+1) \Gamma(-\ell) \Gamma(-\ell+2 i m \gamma)} x^{-\ell-1}\right),
\end{aligned}
$$

where in the last step we used an analytic continuation of the hypergeometric function at spatial infinity and retained only the leading asymptotics. Assuming that $\ell \in \mathbb{R}$, we can extract the SRCs just like in eq. (3.53) and find the same expression (3.55). However, in contrast to the advanced Kerr coordinates, the part of the solution containing the power $r^{-\ell-1}$ does not get canceled by the graviton corrections to the source even in the physical case $\ell \rightarrow \mathbb{N}$. This happens due to the presence of the prefactor $\left(\frac{x}{1+x}\right)^{i m \gamma}$.

We see that the coefficient in front of the power $r^{-\ell-1}$ depends on a choice of coordinates in the physical case $\ell \rightarrow \mathbb{N}$. If we were to use the naive Newtonian matching, we 
would find coordinate-dependent SRCs. The agreement between the different coordinate systems is restored if we use the analytic continuation $\ell \rightarrow \mathbb{R}$.

Finally, we note that non-vanishing of SRCs for Kerr black holes was, in fact, first discovered by Press in 1972 [53], who also argued that they had to be purely imaginary in order for the solution to satisfy the complex regularity condition at the black hole horizon. ${ }^{13}$ Moreover, Press (1972) has also shown that the SRCs capture the spin down produced by the perturbing scalar field. A similar connection was recently discussed in Le Tiec et al. (2020) [25] in the context of gravitational perturbations.

\subsection{Matching to the EFT}

To match the microscopic and the EFT calculation we need to compare the coefficients in front of the $1 / r^{\ell+1}$ power from the two calculations. We have

$$
B_{\ell} n^{\langle L\rangle} \lambda_{L}^{L^{\prime}} \mathcal{E}_{L^{\prime}}=r_{s}^{2 \ell+1} \sum_{m=-\ell}^{\ell} \mathcal{E}_{\ell m}^{(0)} Y_{\ell m} k_{\ell m}^{(0)}
$$

Rewriting the r.h.s. of this equation in the basis of the constant STF tensors $\mathscr{Y}_{\ell m}^{L}$ on $\mathbb{S}^{2}$ as $[41]$

$$
\sum_{m=-\ell}^{\ell} \mathcal{E}_{\ell m}^{(0)} Y_{\ell m} k_{\ell m}^{(0)}=\sum_{m=-\ell}^{\ell} k_{\ell m}^{(0)} \mathscr{Y}_{\ell m}^{L *} n^{\langle L\rangle} \frac{4 \pi \ell !}{(2 \ell+1) ! !} \mathscr{Y}_{\ell m}^{L^{\prime}} \mathcal{E}_{L^{\prime}}^{(0)}
$$

we arrive at the following equation

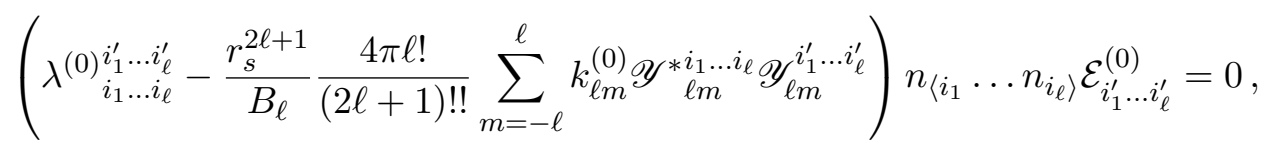

where we have restored the explicit tensorial indices. In what follows we will refer to the constant STF tensors $\mathscr{Y}_{\ell m}^{L}$ as "Thorne tensors" [41]. They allow us to write the following

\footnotetext{
${ }^{13}$ Note that the results presented in that work seem to be affected by an insignificant typo ref. [53] presents the following solution to the radial models of Klein-Gordon equation in the Kerr background (their eq. (10))
}

$$
\text { Press'72: } \quad R_{\ell m}=\left(r-r_{-}\right)^{-i m \gamma}\left(r-r_{+}\right)_{2}^{i m \gamma} F_{1}\left(\ell+1,-\ell, 1+2 i m \gamma ;\left(r-r_{-}\right) /\left(r_{+}-r_{-}\right)\right),
$$

which, in fact, does not satisfy the radial Teukolsky equation [49]. The actual solution may be written in one of the following equivalent forms

$$
\begin{aligned}
R_{\ell m} & =\left(r-r_{-}\right)^{-i m \gamma}\left(r-r_{+}\right)_{2}^{i m \gamma} F_{1}\left(\ell+1,-\ell, 1-2 i m \gamma ;\left(r-r_{-}\right) /\left(r_{+}-r_{-}\right)\right), \\
& =\left(r-r_{-}\right)^{-i m \gamma}\left(r-r_{+}\right)_{2}^{i m \gamma} F_{1}\left(\ell+1,-\ell, 1+2 i m \gamma ;\left(r_{+}-r\right) /\left(r_{+}-r_{-}\right)\right),
\end{aligned}
$$

which differs from eq. (3.64) either by the argument or by the sign in front of the complex part of the third order parameter of the hypergeometric function. This typo has resulted in a sign difference for response coefficients compared to our result. Correcting this typo, eqs. (10), (15c) of Press (1972) imply

$$
\text { Press'72: }\left.\quad \operatorname{Im} R_{\ell m}\right|_{\ell=1, m=1}=-\frac{a}{3}\left(\frac{M}{r}\right)^{2} \quad \text { as } \quad r \rightarrow \infty,
$$

which coincides with our expression (3.63) at linear order in $a$. 
expression for the scalar response tensor

$$
\lambda^{(0)^{i_{1}^{\prime} \ldots i_{\ell}^{\prime}}}=\frac{r_{s}^{2 \ell+1}}{B_{\ell} \ldots i_{\ell}} \frac{4 \pi \ell !}{(2 \ell+1) ! !} \sum_{m=-\ell}^{\ell} k_{\ell m}^{(0)} \mathscr{Y}_{\ell m}^{* i_{1} \ldots i_{\ell}} \mathscr{Y}_{\ell m}^{i_{1}^{\prime} \ldots i_{\ell}^{\prime}}
$$

which is valid up to terms antisymmetric in $i_{1}^{\prime} \ldots i_{\ell}^{\prime}$ and $i_{1} \ldots i_{\ell}$, and up to a Kronecker delta in any combination of $i_{p}$ and $i_{q}^{\prime}$. Note that the expression (3.68) does not impose any restrictions on the symmetry properties of $\lambda^{(0)} L^{\prime}$ w.r.t. multi-index exchange $L \leftrightarrow L^{\prime}$. Hence, our matching procedure based on the expression (3.65) computes both dissipative and conservative responses.

If the Newtonian SRCs $k_{\ell m}^{(0)}$ do not depend on the magnetic number $m$, i.e. $k_{\ell m}^{(0)}=k_{\ell}^{(0)}$, which is the case for Schwarzschild black holes, then the sum over the STF tensors can be explicitly taken, $\frac{4 \pi \ell !}{(2 \ell+1) ! !} \sum_{m=-\ell}^{\ell} \mathscr{Y}_{\ell m}^{* L} \mathscr{Y}_{\ell m}^{L^{\prime}}=\delta_{L L^{\prime}}$. In this case we reproduce the expression for SLNs obtained in Hui et al. [7] for the Schwarzschild black holes (upon identification $\hat{L} \rightarrow \ell$ and $D \rightarrow 4)$

$$
\lambda^{(0)}{ }_{L^{\prime}}^{L}=\lambda_{\ell} \delta_{L^{\prime}}^{L}, \quad \lambda_{\ell}=(-1)^{\ell} \frac{\pi^{1 / 2} \Gamma(1 / 2-\ell)}{2^{\ell-2}} \frac{\Gamma(-2 \ell-1) \Gamma(\ell+1)^{2}}{\Gamma(2 \ell+1) \Gamma(-\ell)^{2}} r_{s}^{2 \ell+1}
$$

These response coefficients vanish identically in the physical case $\ell \in \mathbb{N}$.

Let us now explicitly compute the response matrix (3.68) for the $\ell=1$ and $\ell=2$ sectors. Using formulas from appendix A, we obtain the following expression for $\ell=1$

$$
\lambda^{(0)}{ }_{j}^{i}=\frac{a r_{s}^{2}}{12 B_{1}}\left(\begin{array}{ccc}
0 & 1 & 0 \\
-1 & 0 & 0 \\
0 & 0 & 0
\end{array}\right)
$$

where for simplicity we have retained only the terms linear in black hole's spin $a$. This matrix is antisymmetric, which means that the corresponding dipole worldline coupling vanishes because it contracts two gradients of the scalar field. Hence, the EFT Love tensor is zero in this case even though the Newtonian response coefficients are not. Thus, the Kerr black hole's response is purely dissipative.

Now let us consider the quadrupolar sector $\ell=2$. The corresponding Love tensor computed from eq. (3.68) takes the following form

$$
\lambda^{(0)}{ }_{k l}^{i j}=-(4 \pi) \frac{\chi M^{5}}{135}\left[4\left(1-\chi^{2}\right)^{2} I_{i j, k l}^{(1)}+5 \chi^{2}\left(1-\chi^{2}\right)^{2} I_{i j, k l}^{(3)}+\chi^{4} I_{i j, k l}^{(5)}\right],
$$

where we have introduced the dimensionless black hole spin $\chi=a / M$ and used the following real-valued tensors (defined for any $n$ )

$$
I_{L, L^{\prime}}^{(2 n-1)} \equiv \frac{8 \pi \ell !}{(2 \ell+1) ! !} \sum_{m=-\ell}^{\ell} m^{2 n-1} \operatorname{Im}\left(\mathscr{Y}_{L}^{* \ell m} \mathscr{Y}_{L^{\prime}}^{\ell m}\right)=\frac{1}{2}\left(\begin{array}{ccc}
-2^{2 n-1} \mathbf{M}_{12} & 2^{2 n-1} \mathbf{M}_{11}-\mathbf{M}_{23} \\
2^{2 n-1} \mathbf{M}_{11} & 2^{2 n-1} \mathbf{M}_{12} & \mathbf{M}_{13} \\
-\mathbf{M}_{23} & \mathbf{M}_{13} & \mathbf{0}
\end{array}\right)
$$


which are composed of the STF basis matrices given by $[23,25]$

$$
\mathbf{M}_{11}=\left(\begin{array}{ccc}
1 & 0 & 0 \\
0 & -1 & 0 \\
0 & 0 & 0
\end{array}\right), \quad \mathbf{M}_{12}=\left(\begin{array}{lll}
0 & 1 & 0 \\
1 & 0 & 0 \\
0 & 0 & 0
\end{array}\right), \quad \mathbf{M}_{13}=\left(\begin{array}{lll}
0 & 0 & 1 \\
0 & 0 & 0 \\
1 & 0 & 0
\end{array}\right), \quad \mathbf{M}_{23}=\left(\begin{array}{lll}
0 & 0 & 0 \\
0 & 0 & 1 \\
0 & 1 & 0
\end{array}\right),
$$

and $\mathbf{0}$ is a trivial $3 \times 3$ matrix. For small spin the response matrix takes the following simplified form:

$$
\lambda_{k l}^{(0)}{ }_{k l}^{i j}=-\frac{4 \pi}{3} \frac{\gamma r_{s}^{5}}{180} I_{i j, k l}^{(1)}+\mathcal{O}\left(\gamma^{2}\right)=-(4 \pi) \frac{4 \chi M^{5}}{135} I_{i j, k l}^{(1)}+\mathcal{O}\left(\chi^{2}\right) .
$$

We observe that the tensor $\lambda^{(0)^{i j}}$ is antisymmetric w.r.t. the upper and lower groups of indices, i.e. $\lambda^{(0)^{i j}}=-\lambda^{(0)}{ }_{i j}^{k l}$. This means that the local quadrupole worldline operator vanishes as well. This result can be extended to higher order multipoles.

The antisymmetry of the corresponding matrices stems from the fact that the scalar response numbers are purely imaginary. To see this, let us consider the following general ansatz for response coefficients consistent with the reality requirement for the scalar field multipole expansion (3.1) [25]:

$$
k_{\ell m}^{(0)}=k_{\ell 0}+\chi \sum_{n=1}^{\infty} k_{\ell n}(\chi)(i m)^{n}
$$

where $k_{\ell n}$ are real spin-dependent functions. Plugging this into eq. (3.68) we obtain:

$$
\lambda_{L}^{(0) L^{\prime}}=\frac{r_{s}^{2 \ell+1}}{A_{1}^{\ell}}\left(k_{\ell 0} \delta_{L^{\prime}}^{L}+\chi \sum_{n=1}^{\infty}(-1)^{n}\left[k_{\ell(2 n-1)} I_{L, L^{\prime}}^{(2 n-1)}+k_{\ell(2 n)} R_{L, L^{\prime}}^{(2 n)}\right]\right),
$$

where $I_{L, L^{\prime}}$ are the antisymmetric tensors (w.r.t. exchange $L \leftrightarrow L^{\prime}$ ) introduced in eq. (3.72), whereas $R_{L, L^{\prime}}$ are new fully symmetric tensors defined as follows [25]:

$$
R_{L, L^{\prime}}^{(2 n)} \equiv \frac{8 \pi \ell !}{(2 \ell+1) ! !} \sum_{m=-\ell}^{\ell} m^{2 n} \operatorname{Re}\left(\mathscr{Y}_{L}^{* \ell m} \mathscr{Y}_{L^{\prime}}^{\ell m}\right)=\frac{1}{2}\left(\begin{array}{ccc}
2^{2 n} \mathbf{M}_{11} & 2^{2 n} \mathbf{M}_{12} & \mathbf{M}_{13} \\
2^{2 n} \mathbf{M}_{12} & -2^{2 n} \mathbf{M}_{11} & \mathbf{M}_{23} \\
\mathbf{M}_{13} & \mathbf{M}_{23} & \mathbf{0}
\end{array}\right) .
$$

Comparing eq. (3.75) with eq. (3.76) we see that the real part of the response coefficient generates a symmetric part of $\lambda^{(0)}$ (i.e. even w.r.t. $L \leftrightarrow L^{\prime}$ ), and eventually contributes to the symmetric Love tensor implying non-trivial local EFT operators. However, the imaginary part of the response coefficients generates an antisymmetric part of the response,

$$
I_{L^{\prime}} \propto \kappa_{L^{\prime} L} \mathcal{E}_{L}, \quad \kappa_{L^{\prime} L}=-\kappa_{L L^{\prime}}
$$

which can be identified with a quasi-local contribution given in eq. (3.43). Since the response that we have found is purely imaginary, we conclude that (a) the Love numbers vanish identically, (b) the tidal response of Kerr black holes to static scalar perturbations is entirely dissipative. Note that our antisymmetric quadrupolar response tensor (3.71) coincides (up to a numerical factor) with the tensors that describe the black hole's torque obtained in refs. [25, 27]. 
Finally, we compare the first corrections to the source due to the graviton interaction. We will focus on the first $r_{s} / r$ correction and the first non-trivial spin contribution. Taylor expanding eq. (3.63) we obtain

$$
\Phi \subset r^{\ell}\left(1-\frac{\ell}{2} \frac{r_{s}}{r}+\frac{a^{2} m^{2}}{(4 \ell-2) r^{2}}+\ldots\right),
$$

which agrees with the EFT calculation (3.37).

\section{Spin-1 response coefficients}

In this section we extend our static response calculation to the spin-1 field and compute the response of a Kerr black hole to a long-wavelength electromagnetic perturbation. Similar calculations were done for Schwarzschild black holes in four dimensions in ref. [60] and in a general number of dimensions in ref. [7]. We will start with the definition of spin-1 response coefficients and Love numbers. Then we will compute the electromagnetic field around the Kerr black hole by means of the Newton-Penrose formalism [61, 62], and extract the vector response coefficients from this solution. Finally, we will match our general relativity calculations to the EFT, which will help us fix the relevant tensor Wilson coefficients. As in the scalar field case, our matching procedure will imply the vanishing of the EFT Love tensor, and hence the spin-1 response will be identified to be purely dissipative.

\subsection{Definition}

EFT Love numbers. The local worldline EFT for the electromagnetic field to zeroth order in metric perturbations is given by the following action [7]

$$
\begin{aligned}
S_{\mathrm{EFT}}^{\mathrm{em}}= & S_{\mathrm{pp}}-\frac{1}{4} \int d^{4} x F_{\mu \nu} F^{\mu \nu} \\
& +\sum_{\ell=1} \frac{1}{2 \ell !} \int d^{4} x \int d \tau \delta^{(4)}(x-x(\tau)) \lambda^{(1)}{ }_{L^{\prime}}^{L}\left(\partial_{\left\langle i_{1}\right.} \ldots \partial_{i_{\ell-1}} E_{\left.i_{\ell}\right\rangle}\right)\left(\partial^{\left\langle i_{1}^{\prime}\right.} \ldots \partial^{i_{\ell-1}^{\prime}} E^{\left.i_{\ell}^{\prime}\right\rangle}\right) \\
& +\sum_{\ell=1} \frac{1}{4 \ell !} \int d^{4} x \int d \tau \delta^{(4)}(x-x(\tau)) \tilde{\lambda}_{L^{\prime}}^{(1)}\left\langle\partial_{\left(i_{1}\right.} \ldots \partial_{i_{\ell-1}} B_{\left.i_{\ell}\right\rangle j}\right)\left(\partial^{\left\langle i_{1}^{\prime}\right.} \ldots \partial^{i_{\ell-1}^{\prime}} B^{\left.i_{\ell}^{\prime}\right\rangle j}\right),
\end{aligned}
$$

where $F_{\mu \nu} \equiv 2 \partial_{[\mu} A_{\nu]}$ is the Maxwell tensor, $A_{\mu}$ is the U(1) gauge potential, $S_{\mathrm{pp}}$ is the usual point-particle action (3.8), and we have introduced the electric field vector and the magnetic tensor as

$$
E_{i}=F_{0 i}, \quad B_{i j}=F_{i j} .
$$

They can be defined in a manifestly covariant way by means of the body's 4-velocity and the projector $P_{\mu}^{\nu}=\delta_{\mu}^{\nu}+v_{\mu} v^{\nu}$,

$$
E_{\nu}=F_{\mu \nu} v^{\mu}, \quad B_{\mu \nu}=P_{\mu}^{\sigma} P_{\nu}^{\rho} F_{\sigma \rho} .
$$

The Wilson coefficients $\lambda^{(1)} L_{L^{\prime}}$ and $\tilde{\lambda}_{L^{\prime}}^{(1)} L_{\text {are }}$ the electric and magnetic Love tensors, respectively. In the static limit in the particle's rest frame

$$
E_{i}=F_{\mu i} v^{\mu}=-\partial_{i} A_{0},
$$


which implies that the EFT for the electric field only depends on a scalar field $A_{0}$, and effectively it reduces to the EFT for a massless scalar field that we have studied in the previous section. In order to study the magnetic field it is convenient to employ the transverse gauge $\partial_{i} A_{i}=0$, in which case the kinetic term for the electromagnetic field takes the following form in the zero-frequency limit

$$
-\frac{1}{4} \int d^{4} x F^{\mu \nu} F_{\mu \nu} \rightarrow \frac{1}{2} \int d^{4} x\left[\left(\partial_{i} A_{0}\right)^{2}-\partial_{i} A_{j} \partial_{i} A_{j}\right] .
$$

Definition à la Newtonian matching. It is also useful to introduce a definition of electromagnetic response coefficients in the way similar to the gravitational potential in the Newtonian approximation. This will prove convenient to extract the response coefficients from the general relativity solution that we will obtain in the harmonic space. To that end we can use the fact that in the static limit the electric field is fixed by $A_{0}$. The equation of motion for $A_{0}$ reduces to the Poisson equation in the long-distance limit,

$$
\nabla_{i}^{2} A_{0}=0
$$

In the static limit $A_{0}$ becomes gauge-independent, ${ }^{14}$ and hence we can use it to define response coefficients just like in the case of the Newtonian gravitational potential. Since $A_{0}$ transforms as a scalar under rotations, it can be written as a series over the scalar spherical harmonics ${ }^{15}$

$$
A_{0}=\sum_{\ell=1} \sum_{m=-\ell}^{\ell} Y_{\ell m} \alpha_{\ell m} r^{\ell}\left[1+k_{\ell m}^{(1)}\left(\frac{r}{r_{s}}\right)^{-2 \ell-1}\right],
$$

where $\alpha_{\ell m}$ are source harmonic coefficients, which satisfy $\alpha_{\ell m}^{*}=(-1)^{m} \alpha_{\ell(-m)}$ such that $A_{0}$ is real. Hence, we can use an analog of the Newtonian matching supplemented with the analytic continuation $\ell \rightarrow \mathbb{R}$ to extract electric response coefficients. Note that we have neglected the background electric monopole contribution because we consider neutral black holes in this paper.

One can define the magnetic response using an expansion for the vector part of the gauge potential similar to (4.7). It is easiest to do that at the level of the angular component of the electromagnetic tensor [7]

$$
F_{a b}=2 \nabla_{[a} A_{b]}=2 \sum_{\ell=1} \sum_{m=-\ell}^{\ell} \nabla_{[a} Y_{b]}^{\mathrm{RW}} \frac{\beta_{\ell m}}{\sqrt{\ell(\ell+1)}} r^{\ell+1}\left[1-\frac{\ell+1}{\ell} \tilde{k}_{\ell m}^{(1)}\left(\frac{r}{r_{s}}\right)^{-2 \ell-1}\right]
$$

where $[a, b]$ denotes antisymmetrization, $\beta_{\ell m}$ are magnetic source coefficients, satisfying $\beta_{\ell m}^{*}=(-1)^{m} \beta_{\ell(-m)}$, and $Y_{b \ell m}^{\mathrm{RW}}$ are the Regge-Wheeler transverse vector spherical harmonics [63], see appendix A for detail. The normalization factor $-(\ell+1) / \ell$ is inserted for

\footnotetext{
${ }^{14}$ Indeed, the relevant $\mathrm{U}(1)$ gauge transformation $A_{\mu} \rightarrow A_{\mu}+\partial_{\mu} \alpha$ does not alter $A_{0}$ in the stationary limit $\partial_{t} \rightarrow 0$.

${ }^{15}$ Recall that $A_{0}$ and $A_{r}$ transform as scalars under the $\mathrm{SO}(3)$ group transformations, whereas the vector $A_{a}(a=(\theta, \phi))$ has two distinctive contributions, which transform as a scalar and as a vector under $\mathrm{SO}(3)[7]$.
} 
convenience. With this factor the multipole expansion for the magnetic field $B^{i}=\frac{1}{2} \epsilon^{i j k} F_{j k}$ and the electric field $E^{i}$ take very similar forms, e.g. for the radial component we have

$$
\left(\begin{array}{c}
E^{r} \\
B^{r}
\end{array}\right)=\sum_{\ell=1} \sum_{m=-\ell}^{\ell}\left(\begin{array}{c}
\ell \alpha_{\ell m} \\
\beta_{\ell m}
\end{array}\right) Y_{\ell m} r^{\ell-1}\left(1-\frac{\ell+1}{\ell}\left(\begin{array}{c}
k_{\ell m}^{(1)} \\
\tilde{k}_{\ell m}^{(1)}
\end{array}\right)\left(\frac{r_{s}}{r}\right)^{2 \ell+1}\right) .
$$

From this expression our convention becomes natural as we expect the magnetic and electric response coefficients to coincide in 4 dimensions due to the electric-magnetic duality [7, 40], $k_{\ell m}^{(1)}=\tilde{k}_{\ell m}^{(1)}$. Hence, we will focus on the electric field in the main body of the paper and present an explicit calculation of the magnetic response in appendix $\mathrm{C}$.

\subsection{Newman-Penrose formalism}

To compute $A_{\mu}$ in the Kerr black hole background, we will work within the NewmanPenrose (NP) formalism [61, 62]. In this formalism, the electromagnetic tensor is represented by 3 complex scalars $\Phi_{0}, \Phi_{1}, \Phi_{2}$ as

$$
F_{\mu \nu}=2\left[\Phi_{1}\left(n_{[\mu} l_{\nu]}+m_{[\mu} m_{\nu]}^{*}\right)+\Phi_{2} l_{[\mu} m_{\nu]}+\Phi_{0} m_{[\mu}^{*} n_{\nu]}\right]+\text { c.c. },
$$

where $l_{\mu}, n_{\mu}, m_{\mu}\left(m_{\mu}^{*}\right.$ is the complex conjugate of $\left.m_{\mu}\right)$ are the so-called Newman-Penrose null tetrades. Their explicit expressions in the Boyer-Lindquist coordinates are given in Kinnersley (1969) [64],

$$
\begin{aligned}
\ell^{\mu} & =\left(\frac{\left(r^{2}+a^{2}\right)}{\Delta}, 1,0, \frac{a}{\Delta}\right), \quad n^{\mu}=\left(\frac{r^{2}+a^{2}}{2 \Sigma},-\frac{\Delta}{2 \Sigma}, 0, \frac{a}{2 \Sigma}\right) \\
m^{\mu} & =\frac{1}{\sqrt{2}(r+i a \cos \theta)}\left(i a \sin \theta, 0,1, \frac{i}{\sin \theta}\right) .
\end{aligned}
$$

The quantities $\Phi_{0}, \Phi_{1}, \Phi_{2}$ will be referred to as Maxwell-Newmann-Penrose (MNP) scalars in what follows. The components that we will need for the Newtonian matching are $F_{0 r}$ and $F_{\theta \phi}$. They are given by

$$
\begin{aligned}
F_{0 r}= & -2 \operatorname{Re} \Phi_{1}+\frac{a \sin \theta\left(a \cos \theta \operatorname{Re} \Phi_{0}+r \operatorname{Im} \Phi_{0}\right)}{\sqrt{2} \Sigma}+\frac{a \sin \theta \sqrt{2}\left(a \cos \theta \operatorname{Re} \Phi_{2}-r \operatorname{Im} \Phi_{2}\right)}{\Delta}, \\
F_{\theta \phi}= & 2 \operatorname{Im} \Phi_{1}\left(a^{2}+r^{2}\right) \sin \theta+\frac{a r \operatorname{Re} \Phi_{0} \sin ^{2} \theta \Delta}{\sqrt{2} \Sigma}-\sqrt{2} a r \operatorname{Re} \Phi_{2} \sin ^{2} \theta \\
& -\sqrt{2} a^{2} \operatorname{Im} \Phi_{2} \sin ^{2} \theta \cos \theta-\frac{a^{2} \operatorname{Im} \Phi_{0} \sin ^{2} \theta \cos \theta \Delta}{\sqrt{2} \Sigma},
\end{aligned}
$$

where $\Delta=r^{2}-2 M r+a^{2}, \Sigma=r^{2}+a^{2} \cos ^{2} \theta$. The quantities $\Phi_{0}$ and $\tilde{\Phi}_{2}$, defined as

$$
\tilde{\Phi}_{2} \equiv \frac{(r-i a \cos \theta)^{2}}{\left(r_{+}-r_{-}\right)^{2}} \Phi_{2}
$$

are separable solutions of the Teukolsky equations for spin weights $s=+1$ and $s=-1$, respectively [48, 49] (see also refs. [65-67]). Assuming that the external source is located at spatial infinity, the leading asymptotic behaviors of these MNP scalars are given by $[49,65]$

$$
\Phi_{0} \sim \Phi_{1} \sim \Phi_{2} \sim r^{\ell-1}+\mathrm{const} \cdot r^{-\ell-2},
$$


where "const" is some calculable constant. Hence, in the asymptotic limit $r \rightarrow \infty$ both the magnetic and electric response coefficients can be extracted from a single MNP scalar $\Phi_{1}$

$$
\left.F_{\theta \phi}\right|_{r \rightarrow \infty}=2 \operatorname{Im} \Phi_{1} r^{2} \sin \theta,\left.\quad F_{0 r}\right|_{r \rightarrow \infty}=-2 \operatorname{Re} \Phi_{1} .
$$

\subsection{From Maxwell-Newman-Penrose scalars to response coefficients}

In order to compute $\Phi_{1}$ for the Kerr metric, we will follow the algorithm proposed in Bičák and Dvořák (1976) [65]. We will use the Maxwell equations rewritten in terms of the Newman-Penrose quantities, which are presented in appendix B. As a first step, we compute $\tilde{\Phi}_{2}$. In the Kerr background it factorizes as

$$
\tilde{\Phi}_{2}=\sum_{\ell=1}^{\infty} \sum_{m=-\ell}^{\ell} a_{\ell m} R_{\ell m}^{(2)}(r){ }_{-1} Y_{\ell m}(\theta, \phi),
$$

where ${ }_{-1} Y_{\ell m}$ are the spin-weighted spherical harmonics with weight -1 , and $a_{\ell m}$ are the source-dependent constant harmonic coefficients. Note that we do not impose any restrictions on $a_{\ell m}$ - they are generic complex numbers because $\Phi_{2}$ is complex. The radial function ${ }^{(2)} R_{\ell m}(r)$ satisfies the $s=-1$ Teukolsky equation [48, 49],

$$
\left(-\ell^{2}-\ell+\frac{\gamma m(\gamma m-i(2 x+1))}{x(x+1)}\right) R_{\ell m}^{(2)}(x)+x(x+1) R_{\ell m}^{\prime \prime(2)}(x)=0,
$$

supplemented with the following smoothness boundary condition at the horizon $\left(r \rightarrow r_{+}\right)[49]$

$$
R_{\ell m}^{\prime \prime(2)}(x)=\text { const } \cdot x^{1+i m \gamma}, \quad \text { as } \quad x \rightarrow 0 .
$$

The relevant solution is given by

$$
R_{\ell m}^{(2)}(x)=\left(\frac{x}{x+1}\right)^{+i \gamma m} x(x+1){ }_{2} F_{1}(\ell+2,1-\ell, 2+2 i \gamma m ;-x),
$$

which can be conveniently written as $R_{\ell m}(x) \equiv\left(\frac{x}{x+1}\right)^{-i \gamma m} y_{\ell m}(x)$ with

$$
y_{\ell m} \equiv\left(\frac{x}{x+1}\right)^{2 i \gamma m} x(x+1){ }_{2} F_{1}(\ell+2,1-\ell, 2+2 i \gamma m ;-x) .
$$

Now we can compute the second MNP scalar $\Phi_{0}$, which also factorizes in the spherical coordinates

$$
\Phi_{0}=\sum_{\ell=1}^{\infty} \sum_{m=-\ell}^{\ell} a_{\ell m} R_{\ell m}^{(0)}(r){ }_{+1} Y_{\ell m}(\theta, \phi),
$$

where ${ }_{+1} Y_{\ell m}$ are the spin-weighted spherical harmonics with weight $s=+1$. The radial part $R_{\ell m}^{(0)}$ can be extracted from $R_{\ell m}^{(2)}$ using the Maxwell equations in the NP formalism (see appendix B for more detail)

$$
R_{\ell m}^{(0)}=\frac{2 r_{s a}^{2}}{\ell(\ell+1)}\left(\frac{d}{d r}+\frac{i a m}{\Delta}\right)^{2} R_{\ell m}^{(2)}=\left(\frac{x}{1+x}\right)^{-i \gamma m} \frac{2}{\ell(\ell+1)} \frac{d^{2}}{d x^{2}} y_{\ell m} .
$$


Once $\Phi_{0}$ and $\Phi_{2}$ are determined, we can use the remaining two Maxwell equations in the NP formalism to extract $\Phi_{1}$. This quantity does not fully factorize in the Kerr background

$$
\begin{aligned}
\Phi_{1}= & \frac{\sqrt{2}\left(r_{+}-r_{-}\right)^{2}}{(r-i a \cos \theta)^{2}} \sum_{\ell=1} \sum_{m=-\ell}^{\ell} \frac{a_{\ell m}}{\ell(\ell+1)}\left(\frac{x}{x+1}\right)^{-i \gamma m} \\
& \cdot\left\{[(\ell+1) \ell]^{1 / 2}\left[\frac{(r-i a \cos \theta)}{r_{s a}} \frac{d}{d x}\left(y_{\ell m}\right)-y_{\ell m}\right] Y_{\ell m}-i a \sin \theta \frac{d}{d x}\left(y_{\ell m}\right) \cdot{ }_{+1} Y_{\ell m}\right\},
\end{aligned}
$$

where we have neglected the monopole contribution that corresponds to a shift of black hole's charge. The leading asymptotic in the limit $x \rightarrow \infty$, which is relevant for the Newtonian matching, reads

$$
\left.\Phi_{1}\right|_{x \rightarrow \infty}=\frac{\sqrt{2}}{x^{2}} \sum_{\ell=1} \sum_{m=-\ell}^{\ell} \frac{a_{\ell m}}{(\ell(\ell+1))^{1 / 2}} \cdot\left[x \frac{d}{d x}\left(y_{\ell m}\right)-y_{\ell m}\right] Y_{\ell m}
$$

As anticipated, $\Phi_{1}$ factorizes in the asymptotic limit $r \rightarrow \infty$. Recall that we are eventually interested in $A_{0}$, which is related to $\Phi_{1}$ via

$$
\partial_{r} A_{0}=-F_{0 r}=2 \operatorname{Re} \Phi_{1}
$$

At this point we can rewrite $a_{\ell m}$ as,

$$
\frac{2 \sqrt{2}}{(\ell(\ell+1))^{1 / 2}} a_{\ell m} r_{s a}=\left(\alpha_{\ell m}+i \beta_{\ell m}\right)
$$

where $\alpha_{\ell m}$ and $\beta_{\ell m}$ satisfy the reality conditions $\alpha_{\ell m}^{*}=(-1)^{m} \alpha_{\ell(-m)}^{*}$ and $\beta_{\ell m}^{*}=(-1)^{m} \beta_{\ell(-m)}^{*}$. The harmonic coefficients $\alpha_{\ell m}$ and $\beta_{\ell m}$ capture electric and magnetic parts of the Maxwell tensor, respectively. To obtain $A_{0}$, we integrate eq. (4.23) as follows

$$
\begin{aligned}
A_{0} & =2 \operatorname{Re} \int^{r} d r^{\prime} \Phi_{1}\left(r^{\prime}\right)=\operatorname{Re} \sum_{\ell=1} \sum_{m=-\ell}^{\ell} \frac{2 \sqrt{2} a_{\ell m} r_{s a}}{(\ell(\ell+1))^{1 / 2}} Y_{\ell m} \int^{x} \frac{d x^{\prime}}{x^{\prime 2}}\left[x^{\prime} \frac{d}{d x^{\prime}}\left(y_{\ell m}\left(x^{\prime}\right)\right)-y_{\ell m}\left(x^{\prime}\right)\right] \\
& =\sum_{\ell=1} \sum_{m=-\ell}^{\ell} Y_{\ell m} \alpha_{\ell m} \frac{y_{\ell m}}{x}=\sum_{\ell=1} \sum_{m=-\ell}^{\ell} \alpha_{\ell m} A_{\ell m}^{(0)}(r) Y_{\ell m},
\end{aligned}
$$

where in the last line we used that the sum $\sum_{\ell m} \alpha_{\ell m} Y_{\ell m} y_{\ell m}$ is real, which is a consequence of $\alpha_{\ell m}^{*}=\alpha_{\ell(-m)}, Y_{\ell m}^{*}=(-1)^{m} Y_{\ell(-m)}$, and $y_{\ell m}^{*}=y_{\ell(-m)}$ (see eq. (4.19)). Expanding the radial mode functions at spatial infinity we obtain

$$
\begin{aligned}
A_{\ell m}^{(0)}(r) & =\text { const } \cdot(x+1)_{2} F_{1}(\ell+2,1-\ell, 2+2 i \gamma m,-x) \\
& \underset{x \rightarrow \infty}{\longrightarrow} r_{s a}^{\ell} x^{\ell}\left(1+\frac{\Gamma(-2 \ell-1) \Gamma(\ell+2) \Gamma(\ell+2 i m \gamma+1)}{\Gamma(1-\ell) \Gamma(2 \ell+1) \Gamma(-\ell+2 i m \gamma)} x^{-2 \ell-1}\right) .
\end{aligned}
$$


Now we can compare this result with the large-distance approximation (4.7) and read off the following electromagnetic response coefficients

$$
\begin{aligned}
k_{\ell m}^{(1)} & =\frac{\Gamma(-2 \ell-1) \Gamma(\ell) \Gamma(\ell+2 i m \gamma+1)}{\Gamma(-1-\ell) \Gamma(2 \ell+1) \Gamma(-\ell+2 i m \gamma)}\left(\frac{r_{s a}}{r_{s}}\right)^{2 \ell+1} \\
& =i m \gamma \frac{(\ell+1) !(\ell-1) !}{(2 \ell) !(2 \ell+1) !} \prod_{n=1}^{\ell}\left(n^{2}+4 m^{2} \gamma^{2}\right)\left(\frac{r_{s a}}{r_{s}}\right)^{2 \ell+1} \\
& =\frac{i m \chi}{2} \frac{(\ell+1) !(\ell-1) !}{(2 \ell) !(2 \ell+1) !} \prod_{n=1}^{\ell}\left(n^{2}\left(1-\chi^{2}\right)+m^{2} \chi^{2}\right),
\end{aligned}
$$

where we replaced $\Gamma(\ell+2) / \Gamma(1-\ell) \rightarrow \Gamma(\ell) / \Gamma(-\ell-1)$ in the first line, then assumed the physical values $\ell \in \mathbb{N}$, and finally used eq. (A.18). As in the scalar case, the electromagnetic response coefficients are purely imaginary, which means that the static Love numbers must vanish. We will confirm that shortly.

It is instructive to take the limit $\gamma \rightarrow 0$ and compare our resulting expression with the Schwarzschild black hole electromagnetic Love numbers $k_{S}$ computed in ref. [7]. This work defined electromagnetic Love numbers w.r.t. the scalar mode $\Psi_{S}$, defined as

$$
\Psi_{S}=\frac{r^{2}}{\sqrt{\ell(\ell+1)}} \partial_{r} A_{0}
$$

in four dimensions. This means that we need to differentiate $A_{0}$ w.r.t. the radial coordinate $r$ to obtain $\Psi_{S}$, which produces an additional factor $-(\ell+1) / \ell$ in front of the Love number. With this factor taken into account, we have

$$
\begin{aligned}
k_{S} \equiv-\left.\frac{(\ell+1)}{\ell} k_{\ell m}^{(1)}\right|_{\gamma=0} & =-\frac{(\ell+1)}{\ell} \frac{\Gamma(-2 \ell-1) \Gamma(\ell+2) \Gamma(\ell+1)}{\Gamma(1-\ell) \Gamma(2 \ell+1) \Gamma(-\ell)} \\
& =\frac{\Gamma(-2 \ell-1) \Gamma(\ell+2) \Gamma(\ell)}{\Gamma(1-\ell) \Gamma(2 \ell+1) \Gamma(-\ell-1)},
\end{aligned}
$$

where we have used $\Gamma(x) x=\Gamma(x+1)$. This expression exactly coincides with the electromagnetic Love numbers given in ref. [7] after the identification $\hat{L} \rightarrow \ell$ and $D \rightarrow 4$. It vanishes once we take the physical limit $\ell \rightarrow \mathbb{N}$.

\subsection{Matching to the EFT}

In this section, we perform an explicit matching of the worldline point-particle effective field theory that includes electromagnetism to the results of the full GR calculation. This will allow us to extract the electric polarizability operator in the EFT from the electric response coefficients that we have previously found in this section. The calculation of the magnetic susceptibilities can be easily performed in the same fashion. We present this calculation in appendix $\mathrm{C}$ for completeness.

The calculation of the electric Love numbers is identical to the scalar field Love number matching. Introducing an external background source as

$$
\bar{A}_{0}=\bar{\alpha}_{i_{1} \ldots i_{\ell}} x^{i_{1}} \ldots x^{i_{\ell}}
$$


where $\bar{\alpha}_{i_{1} \ldots i_{\ell}}$ is an STF tensor, and solving the equation of motion for $A_{0}$ just like in the scalar field case we obtain

$$
A_{0}=\sum_{\ell m} \bar{\alpha}_{\ell m} r^{\ell} Y_{\ell m}+\lambda^{(1)}{ }_{L^{\prime}}^{L} \bar{\alpha}_{L} n^{L^{\prime}}(-1)^{\ell+1} \frac{2^{\ell-2}}{\pi^{1 / 2} \Gamma(1 / 2-\ell)} r^{-\ell-1} .
$$

In principle, we could match directly eq. (4.25) and eq. (4.31) as $A_{0}$ is gauge-independent in the static limit. However, generally it is more appropriate to match the components of the electric tensor, such as $E_{r}$, in order to ensure that the result in gauge-independent. Acting on eqs. (4.25) and eq. (4.31) with one derivative w.r.t. the radial coordinate $r$, matching the two results, and rewriting the sum over the spherical harmonics in terms of the Thorne STF tensors we obtain the following expression for the electromagnetic response matrix

$$
\lambda^{(1)}{ }_{i_{1} \ldots i_{\ell}}^{i_{1}^{\prime} \ldots i_{\ell}^{\prime}}=-\frac{r_{s}^{2 \ell+1}}{B_{\ell}} \frac{4 \pi \ell !}{(2 \ell+1) ! !} \sum_{m=-\ell}^{\ell} k_{\ell m}^{(1)} \mathscr{Y}_{\ell m}^{* i_{1} \ldots i_{\ell}} \mathscr{Y}_{\ell m}^{i_{1}^{\prime} \ldots i_{\ell}^{\prime}}
$$

where $B_{\ell}$ is a constant given in eq. (3.31). As in the scalar case, we see that the requirement that $\lambda^{(1)} L_{L}^{\prime}$ is even w.r.t. exchange $L \leftrightarrow L^{\prime}$ has disappeared in the expression (4.32), and hence we can interpret it as a general expression for response coefficients that includes conservative and dissipative effects on the same footing.

Plugging $\gamma=0$, we find that this expression reduces the Love numbers for the Schwarzschild black holes given in eq. (5.30) of ref. [7] (upon identification $\hat{L} \rightarrow \ell, D \rightarrow 4$, and up to a sign),

$$
\begin{aligned}
\lambda^{(1)} L_{L^{\prime}}^{L} & =\lambda_{\ell}^{(E)} \delta_{L^{\prime}}^{L}, \quad \text { where } \\
\lambda_{\ell}^{(E)} & =(-1)^{\ell+1} \frac{\pi^{1 / 2} \Gamma(1 / 2-\ell)}{2^{\ell-2}} \frac{\Gamma(-2 \ell-1) \Gamma(\ell+2) \Gamma(\ell+1)}{\Gamma(1-\ell) \Gamma(2 \ell+1) \Gamma(-\ell)} r_{s}^{2 \ell+1} .
\end{aligned}
$$

This Wilson coefficient vanishes for physical values of the orbital number $\ell \in \mathbb{N}$.

Now let us get back to the expression for the electromagnetic response tensor (4.32). As in the scalar case, we see that the electric response tensors are antisymmetric for all $\ell$ 's as a result of vanishing of the real part of $k_{\ell m}^{(1)}$. For instance, in the quadrupolar sector we have

$$
\begin{aligned}
\lambda_{k l}^{(1)} \underset{k j}{i j} & =-(4 \pi) \frac{\chi M^{5}}{45} \frac{1}{2}\left[4\left(1-\chi^{2}\right)^{2} I_{i j, k l}^{(1)}+5 \chi^{2}\left(1-\chi^{2}\right)^{2} I_{i j, k l}^{(3)}+\chi^{4} I_{i j, k l}^{(5)}\right] \\
& =-\frac{4 \pi}{3} \frac{\gamma r_{s}^{5}}{120} I_{i j, k l}^{(1)}+\mathcal{O}\left(\gamma^{2}\right)=-(4 \pi) \frac{2 \chi M^{5}}{45} I_{i j, k l}^{(1)}+\mathcal{O}\left(\chi^{2}\right),
\end{aligned}
$$

where we used the dimensionless spin $\chi=a / M$ and took the $\gamma \rightarrow 0$ limit in the last line. The STF basis tensors $I^{(1)}, I^{(3)}, I^{(5)}$ are defined in eqs. (3.72), (3.73). We see that just like in the scalar case, the local electromagnetic worldline EFT couplings vanish even though the imaginary electric response coefficients do not. We conclude that the spin- 1 response is purely dissipative. 


\section{Spin-2 response coefficients}

For completeness, in this section we present the computation of the static response of Kerr black hole to the external gravitational perturbation. This calculation has been discussed in detail in refs. $[25,26]$, and some important technical results were previously obtained in refs. [43, 68]. Our main novel result here will be an explicit matching of the spin-2 Kerr black hole response coefficients to the worldline EFT Wilson coefficients along the lines of the previous sections.

\subsection{Definition}

EFT Love numbers. The local worldline EFT of gravitational perturbations is built out of various operators constructed from the Weyl tensor [5, 7]. In four dimensions this tensor has two distinctive components,

$$
E_{\mu \sigma}=C_{\mu \nu \sigma \rho} v^{\nu} v^{\rho}, \quad B_{\mu \nu \sigma}=P_{\mu}^{\mu^{\prime}} P_{\nu}^{\nu^{\prime}} P_{\sigma}^{\sigma^{\prime}} C_{\rho \mu^{\prime} \nu^{\prime} \sigma^{\prime}} v^{\rho} .
$$

In the body's rest frame these components reduce to

$$
E_{i j}^{(2)} \equiv C_{0 i 0 j}, \quad B_{i j k}^{(2)} \equiv C_{0 i j k}
$$

Note that magnetic tensor can also be dualized as $B_{\mu \sigma}=\frac{1}{2} \epsilon_{\mu \alpha \beta \nu} C^{\alpha \beta}{ }_{\sigma \rho} v^{\nu} v^{\rho}$, but here we will not do that in order to match the convention of ref. [7]. The most generic quadratic action for $E_{i j}^{(2)}$ and $B_{i j k}^{(2)}$ is given by

$$
\begin{aligned}
S_{\mathrm{EFT}}^{\mathrm{grav}}= & S_{\mathrm{pp}}+\int d^{4} x h \mathcal{D}^{2} h \\
& +\sum_{\ell=2} \frac{1}{2 \ell !} \int d^{4} x \int d \tau \delta^{(4)}(x-x(\tau)) \lambda^{(2)}{ }_{L}^{L^{\prime}} \partial_{\left\langle i_{1}\right.} \ldots \partial_{i_{\ell-2}} E_{\left.i_{\ell-1} i_{\ell}\right\rangle}^{(2)} \partial^{\left\langle i_{1}^{\prime}\right.} \ldots \partial^{i_{\ell-2}^{\prime}} E^{\left.(2)^{i_{\ell-1}^{\prime}} i_{\ell}^{\prime}\right\rangle} \\
& +\sum_{\ell=2} \frac{1}{4 \ell !} \int d^{4} x \int d \tau \delta^{(4)}(x-x(\tau)) \tilde{\lambda}^{(2)}{ }_{L}^{L^{\prime}} \partial_{\left\langle i_{1}\right.} \ldots \partial_{i_{\ell-2}} B_{\left.i_{\ell-1} i_{\ell}\right\rangle j}^{(2)} \partial^{\left\langle i_{1}^{\prime}\right.} \ldots \partial^{i_{\ell-2}^{\prime}} B^{\left.(2)^{i_{\ell-1}^{\prime}} i_{\ell}^{\prime}\right\rangle j},
\end{aligned}
$$

where $\int d^{4} x h \mathcal{D}^{2} h$ denotes the graviton kinetic term, whose explicit expression can be found e.g. in refs. [7, 54]. The tensorial Wilson coefficients $\lambda^{(2)}{ }_{L}^{L^{\prime}}$ and $\tilde{\lambda}_{L}^{(2)}{ }_{L}^{L^{\prime}}$ will be referred to as the electric and magnetic spin-2 Love tensors, respectively.

Response coefficients in the Newtonian limit. The general spin-2 tidal response coefficients can be related to the harmonic expansion of the Newtonian potential in the large distance limit. Their calculation relies on the curvature Weyl scalar, defined as

$$
\psi_{0} \equiv C_{\alpha \beta \gamma \delta} l^{\alpha} m^{\beta} l^{\gamma} m^{\delta},
$$

where $C_{\alpha \beta \gamma \delta}$ is the Weyl tensor projected onto the Newman-Penrose null tetrades [61, 62]. In the Newtonian limit the Weyl scalar takes the following form,

$$
\psi_{0}=-2 m^{i} m^{j} \nabla_{i} \nabla_{j} U
$$


where $U$ is the Newtonian potential, and $\nabla_{i}$ is covariant derivative of the 3 -dimensional euclidean spacetime. Plugging the expression for the Newtonian potential (2.12) into eq. (5.5), we find

$$
\left.\psi_{0}\right|_{r \rightarrow \infty}=\sum_{\ell=2}^{\infty} \sum_{m=-\ell}^{\ell} \sqrt{\frac{(\ell+2)(\ell+1)}{\ell(\ell-1)}} r^{\ell-2} \mathcal{E}_{\ell m}\left[1+k_{\ell m}\left(\frac{r_{s}}{r}\right)^{2 \ell+1}\right]{ }_{+2} Y_{\ell m}(\theta, \phi),
$$

where ${ }_{+2} Y_{\ell m}$ denotes the $s=+2$ spin-weighted spherical harmonics. In the relativistic regime this expression can be generalized as follows [25, 43, 68]:

$$
\left.\psi_{0}\right|_{r \rightarrow \infty}=\sum_{\ell=2}^{\infty} \sum_{m=-\ell}^{\ell} \sqrt{\frac{(\ell+2)(\ell+1)}{\ell(\ell-1)}} r^{\ell-2}\left(\mathcal{E}_{\ell m}+i \frac{\ell+1}{3} \mathcal{B}_{\ell m}\right)\left[1+k_{\ell m}\left(\frac{r_{s}}{r}\right)^{2 \ell+1}\right]{ }_{+2} Y_{\ell m}(\theta, \phi),
$$

where $\mathcal{E}_{\ell m}, \mathcal{B}_{\ell m}$ are the spherical harmonic coefficients of the electric-type and magnetictype tidal tensors, defined by means of the Weyl tensor as follows:

$$
\mathcal{E}_{L} \equiv \frac{1}{(\ell-2) !} \nabla_{\left\langle i_{3} \ldots i_{\ell}\right.} C_{0\left|i_{1}\right| 0\left|i_{2}\right\rangle}, \quad \mathcal{B}_{L} \equiv \frac{3}{2(\ell-2) !(\ell+1) !} \nabla_{\left\langle i_{3} \ldots i_{\ell}\right.} \epsilon_{j k \mid i_{1}} C_{i_{2}|0 j k\rangle} .
$$

The electric-type tidal tensor is a relativistic generalization of the Newtonian tidal tensor discussed in section 2. Note that the response coefficients are the same for magnetictype and electric-type perturbations as a consequence of the gravitational electric-magnetic duality, which takes place for fluctuations around Kerr black holes in four dimensions [40].

\subsection{From Weyl scalar to response coefficients}

Eq. (5.7) can be used to extract the Newtonian response coefficients from the full general relativity calculation. Indeed, the Weyl scalar $\psi_{0}$ factorizes in the Kerr background as [25]

$$
\psi_{0}=\sum_{\ell=2}^{\infty} \sum_{m=-\ell}^{\ell} \sqrt{\frac{(\ell+2)(\ell+1)}{\ell(\ell-1)}}\left(\mathcal{E}_{\ell m}+i \frac{\ell+1}{3} \mathcal{B}_{\ell m}\right) R_{\ell m}^{s=+2}(r)_{+2} Y_{\ell m}(\theta, \phi)
$$

where the radial function $R_{\ell m}^{s=+2}$ satisfies the following differential equation

$$
\left[\left(-\ell^{2}-\ell+6+\frac{\gamma m(\gamma m+i 2(2 x+1))}{x(x+1)}\right)+3(2 x+1) \frac{d}{d x}+x(x+1) \frac{d^{2}}{d x^{2}}\right] R_{\ell m}^{s=+2}(x)=0 .
$$

The solution smooth at the black hole horizon must satisfy the following boundary condition in the Boyer-Lindquist coordinates [49],

$$
R_{\ell m}^{s=+2}=\text { const } \cdot x^{-2+i m \gamma} \quad \text { as } \quad r \rightarrow r_{+}(x \rightarrow 0) .
$$

The desired radial function is given by

$$
R_{\ell m}^{s=+2}=\text { const } \cdot(1+x)^{-i m \gamma-2} x^{i m \gamma-2}{ }_{2} F_{1}(-\ell-2, \ell-1 ;-1+2 i \gamma m ;-x) .
$$

Taylor-expanding this function at spatial infinity we find

$$
R_{\ell m}^{s=+2}=x^{\ell-2} r_{s a}^{\ell-2}\left(1+x^{-2 \ell-1} \frac{\Gamma(-2 \ell-1) \Gamma(\ell-1) \Gamma(\ell+2 \gamma i m+1)}{\Gamma(-\ell-2) \Gamma(2 i \gamma m-\ell) \Gamma(2 \ell+1)}\right),
$$


which provides us with the following gravitational response coefficients

$$
k_{\ell m} \equiv k_{\ell m}^{(2)}=\frac{\Gamma(-2 \ell-1) \Gamma(\ell-1) \Gamma(\ell+2 \gamma i m+1)}{\Gamma(-\ell-2) \Gamma(2 i \gamma m-\ell) \Gamma(2 \ell+1)}\left(\frac{r_{s a}}{r_{s}}\right)^{2 \ell+1} .
$$

Note that this expression coincides with eqs. $(4.29,4.40)$ of ref. [25]. For the physical case $\ell \in \mathbb{N}$ we have

$$
\begin{aligned}
k_{\ell m}^{(2)} & =-i m \gamma \frac{(\ell-2) !(\ell+2) !}{(2 \ell) !(2 \ell+1) !} \prod_{n=1}^{\ell}\left(n^{2}+4 m^{2} \gamma^{2}\right)\left(\frac{r_{s a}}{r_{s}}\right)^{2 \ell+1} \\
& =-\frac{i m \chi}{2} \frac{(\ell-2) !(\ell+2) !}{(2 \ell) !(2 \ell+1) !} \prod_{n=1}^{\ell}\left(n^{2}\left(1-\chi^{2}\right)+m^{2} \chi^{2}\right),
\end{aligned}
$$

where in the last line we expressed the result in terms of the dimensionless black hole spin $\chi=a / M$. Importantly, the spin-2 response coefficients are purely imaginary just like their spin- 0 and spin- 1 counterparts. This means that the tidal spin-2 Love tensors must vanish identically.

\subsection{Matching to the EFT}

We will focus on the electric part of the spin-2 perturbations captured by $E_{i j}^{(2)} \equiv C_{0 i 0 j}$ in what follows. The calculation of the magnetic part can be carried out in a similar fashion. To match the electric-type Love numbers, it is sufficient to consider only the following scalar graviton modes

$$
g_{00}=-1+2 h_{00}, \quad g_{i j}=\delta_{i j}\left(1+2 \tilde{h}_{00}\right)
$$

which corresponds to Newtonian gauge. In this gauge the gravity kinetic term takes the following form

$$
\int d^{4} x h \mathcal{D}^{2} h=\frac{1}{16 \pi} \int d^{4} x\left[4 h_{00} \Delta \tilde{h}_{00}-2 \tilde{h}_{00} \Delta \tilde{h}_{00}\right] .
$$

The field $\tilde{h}_{00}$ does not appear in $S_{\mathrm{pp}}$ at zeroth order in particle's displacement from the center of mass position. Thus, in this approximation it can be integrated out from the action by means of its equation of motion $\tilde{h}_{00}=h_{00}$, which gives us

$$
\int d^{4} x h \mathcal{D}^{2} h=\frac{1}{8 \pi} \int d^{4} x h_{00} \Delta h_{00} .
$$

Then the electric part of the Weyl tensor takes the following form

$$
E_{i j}^{(2)}=-\partial_{i} \partial_{j} h_{00}
$$

All in all, in the static limit the EFT takes the same form as the EFT for a scalar field, modulo a factor $4 \pi$ in the graviton kinetic term

$$
\begin{aligned}
S_{\mathrm{EFT}}^{\mathrm{grav}}= & S_{\mathrm{pp}}+\frac{1}{8 \pi} \int d^{4} x h_{00} \Delta h_{00} \\
& +\sum_{\ell=2} \frac{1}{2 \ell !} \int d^{4} x \int d \tau \delta^{(4)}(x-x(\tau)) \lambda_{{ }^{(2)}{ }_{1}^{\prime} \ldots i_{\ell}^{\prime}}^{i_{1} \ldots i_{\ell}}\left(\partial_{\left\langle i_{1}\right.} \ldots \partial_{\left.i_{\ell}\right\rangle} h_{00}\right)\left(\partial^{\left\langle i_{1}^{\prime}\right.} \ldots \partial^{\left.i_{\ell}^{\prime}\right\rangle} h_{00}\right),
\end{aligned}
$$


where $S_{\mathrm{pp}}$ is the standard point-particle action (3.8). Repeating the scalar field calculation for a fixed multipolar index $\ell$, we can easily obtain the following static response

$$
h_{00}=\sum_{m=-\ell}^{\ell} \overline{\mathcal{E}}_{\ell m} r^{\ell} Y_{\ell m}+\lambda^{(2)}{ }_{L^{\prime}}^{L} n^{L^{\prime}} \overline{\mathcal{E}}_{L} \cdot(-1)^{\ell} \frac{8 \pi}{2} \frac{2^{\ell-2}}{\pi^{1 / 2} \Gamma(1 / 2-\ell)} r^{-\ell-1},
$$

where $\overline{\mathcal{E}}_{\ell m}$ are spherical modes of the background source and $\overline{\mathcal{E}}_{L}$ is the corresponding constant STF tensor. In order to be rigorous and ensure that the result for response coefficients is gauge-independent, we need to match gauge invariant quantities from both sides. The simplest such quantity is the $r r$ component of the electric part of the Weyl tensor [7],

$$
C_{0 r 0 r}=E_{r r}^{(2)}=-\partial_{r}^{2} h_{00} .
$$

Taking two derivatives w.r.t. $r$ in eq. (5.21) and in the formula for the Newtonian potential (2.12), and matching the two expressions we obtain

$$
\lambda^{(2)}{ }_{i_{1} \ldots i_{\ell}}^{i_{1}^{\prime} \ldots i_{\ell}^{\prime}}=\frac{2 r_{s}^{2 \ell+1}}{8 \pi B_{\ell}} \frac{4 \pi \ell !}{(2 \ell+1) ! !} \sum_{m=-\ell}^{\ell} k_{\ell m}^{(2)} \mathscr{Y}_{\ell m}^{* i_{1} \ldots i_{\ell}} \mathscr{Y}_{\ell m}^{i_{1}^{\prime} \ldots i_{\ell}^{\prime}},
$$

where $k_{\ell m}^{(2)}$ are given in eq. (5.15), $B_{\ell}$ a constant is given in eq. (3.31). ${ }^{16}$

Plugging $\gamma=0$, we find that expression (5.23) coincides with the Love number for the Schwarzschild black holes given in eq. (5.50) of ref. [7] for generic $\ell \in \mathbb{R}$ (upon identification $\hat{L} \rightarrow \ell, D \rightarrow 4$, and modulo the conventional factor $8 \pi$ ). However, this expression vanishes in the physical case $\ell \in \mathbb{N} /\{1\}$,

$$
\begin{aligned}
\lambda^{(2)}{ }_{i_{1} \ldots i_{\ell}}^{i_{1}^{\prime} \ldots i_{\ell}^{\prime}} & =\lambda_{\ell}^{\left(C_{E}\right)} \delta_{\left\langle i_{1} \ldots i_{\ell}\right\rangle}^{\left\langle i_{1}^{\prime} \ldots i_{\ell}^{\prime}\right\rangle}, \\
\lambda_{\ell}^{\left(C_{E}\right)} & =\frac{2}{8 \pi}(-1)^{\ell} \frac{\pi^{1 / 2} \Gamma(1 / 2-\ell)}{2^{\ell-2}} \frac{\Gamma(-2 \ell-1) \Gamma(\ell-1) \Gamma(\ell+1)}{\Gamma(-\ell-2) \Gamma(-\ell) \Gamma(2 \ell+1)} r_{s}^{2 \ell+1}=0 \quad \text { if } \quad \ell \in \mathbb{N} /\{1\} .
\end{aligned}
$$

Using explicit formulas for the Thorne tensors from appendix A, we obtain the following expression for the EFT quadrupolar worldline tensor coupling in terms of the dimensionless spin parameter $\chi=a / M$ :

$$
\lambda_{k l}^{(2)}=-\frac{2 \chi M^{5}}{45}\left[4\left(1-\chi^{2}\right)^{2} I_{i j, k l}^{(1)}+5 \chi^{2}\left(1-\chi^{2}\right)^{2} I_{i j, k l}^{(3)}+\chi^{4} I_{i j, k l}^{(5)}\right],
$$

where the STF basis tensors $I^{(1)}, I^{(3)}, I^{(5)}$ are defined in eqs. (3.72), (3.73). For small spin this expression simplifies as,

$$
\lambda^{(2)}{ }_{k l}^{i j}=-2 \frac{4 \pi}{3(8 \pi)} \frac{\gamma r_{s}^{5}}{30} I_{i j, k l}^{(1)}+\mathcal{O}\left(\chi^{2}\right)=-\frac{8 \chi M^{5}}{45} I_{i j, k l}^{(1)}+\mathcal{O}\left(\chi^{2}\right) .
$$

Just like in the case of spin- 0 and spin- 1 perturbations, the worldline finite-size operators vanish even though the Newtonian response coefficients do not. Note that our expressions for the response matrices (5.25), (5.26) coincide with those presented in Le Tiec

\footnotetext{
${ }^{16}$ Note that the same result can be obtained by a direct matching of $h_{00}$ from (5.21) and the Newtonian potential (2.12) because our choice of Newtonian gauge (5.16) is precisely the one that reproduces the Newtonian limit at large distances.
} 
et al. (2020) [25] and those obtained in Goldberger et al. (2020) [27] (up to a conventional numerical factor). The antisymmetric response captured by these matrices is responsible for the dissipative effect of tidal torques.

\section{Master formula for black hole response coefficients}

In this section we demonstrate that black hole's response coefficients for any perturbing boson field can be extracted directly from Teukolsky equations for relevant Newman-Penrose scalars. Then we will present the response coefficients for time-dependent perturbations.

\subsection{Static responses}

An important observation is that the Kerr black hole response coefficients for all fields can be extracted directly from the solution to the radial Teukolsky equation for a generic spin weight $s[49]$,

$$
\begin{aligned}
{\left[s^{2}+s-\ell^{2}-\ell+\right.} & \frac{(\gamma m)^{2}+i \gamma m s(2 x+1)}{x(x+1)} \\
& \left.+(s+1)(2 x+1) \frac{d}{d x}+x(x+1) \frac{d^{2}}{d x^{2}}\right] R(x)=0 .
\end{aligned}
$$

This solution needs to satisfy the following boundary condition at the future horizon

$$
R=\text { const } \times\left(r-r_{+}\right)^{i \gamma m-s}, \quad \text { as } r \rightarrow r_{+},
$$

which ensures that the in-falling observes sees only the so-called "non-special" fields (= fields that are not singular and not identically equal to zero). Moreover, these boundary condition guarantees that the energy momentum flux flows strictly into the black hole [48, 49, 51]. The desired solution can be easily constructed [69, 70],

$$
R=\text { const } \cdot(1+x)^{-i m \gamma-s} x^{i m \gamma-s}{ }_{2} F_{1}(-\ell-s, \ell+1-s ; 1+2 i \gamma m-s ;-x) .
$$

Taylor-expanding this solution at spatial infinity $x \rightarrow \infty$ (see appendix A for the relevant analytic continuation formula) we find

$$
R=\mathrm{const} \cdot x^{\ell-s} r_{s a}^{\ell}\left(1+x^{-2 \ell-1} \frac{\Gamma(-2 \ell-1) \Gamma(\ell-s+1) \Gamma(\ell+2 \gamma i m+1)}{\Gamma(-\ell-s) \Gamma(2 i \gamma m-\ell) \Gamma(2 \ell+1)}\right),
$$

which provides us with the following dimensionless response coefficients

$$
\begin{aligned}
k_{\ell m}^{(s)} & =\frac{\Gamma(-2 \ell-1) \Gamma(\ell-s+1) \Gamma(\ell+2 \gamma i m+1)}{\Gamma(-\ell-s) \Gamma(2 i \gamma m-\ell) \Gamma(2 \ell+1)}\left(\frac{r_{s a}}{r_{s}}\right)^{2 \ell+1} \\
& =(-1)^{s+1} \frac{i m \chi}{2} \frac{(\ell+s) !(\ell-s) !}{(2 \ell) !(2 \ell+1) !} \prod_{n=1}^{\ell}\left(n^{2}\left(1-\chi^{2}\right)+m^{2} \chi^{2}\right),
\end{aligned}
$$

where $\chi=J / M^{2}$ is black hole's dimensionless spin, and $r_{s}=2 M$ is the Schwarzschild radius. This expression recovers the scalar response coefficients for $s=0$ (see eq. (3.54)), the spin-1 (electromagnetic) response coefficients for $s=1$ (see eq. (4.27)), and the spin-2 (gravitational) response coefficients for $s=2$ (see eq. (5.14)). 


\subsection{Time-dependent responses}

Using the Teukolsky equation, we can also obtain expressions for non-static responses to all orders in frequency, which we present here for completeness. Let us consider a generic field $\psi$ of spin weight $s$ that factorizes in the Kerr background as [48, 49]

$$
\psi=e^{-i \omega t+i m \phi} R(r) S(\theta) .
$$

The functions $R$ and $S$ satisfy the following frequency-dependent equations

$$
\begin{aligned}
& {\left[\frac{\left(\omega\left(a^{2}+r^{2}\right)-a m\right)\left(\omega\left(a^{2}+r^{2}\right)-a m+2 i s(M-r)\right)}{a^{2}+r(r-2 M)}-a^{2} \omega^{2}+2 a m \omega-A+4 i r s \omega\right.} \\
& \left.+\left(a^{2}+r(r-2 M)\right) \frac{d^{2}}{d r^{2}}+2(s+1)(r-M) \frac{d}{d r}\right] R(r)=0, \\
& \frac{1}{\sin \theta} \frac{d}{d \theta}\left(\sin \theta \frac{d S(\theta)}{d \theta}\right)+\left(a^{2} \omega^{2} \cos ^{2} \theta-\frac{m^{2}}{\sin ^{2} \theta}-2 a \omega s \cos \theta\right. \\
& \left.-\frac{2 m s \cos \theta}{\sin ^{2} \theta}-s^{2} \cot ^{2} \theta+s+A-a^{2} \omega^{2}-2 a m \omega\right) S(\theta)=0,
\end{aligned}
$$

where $A$ denotes angular eigenvalues. For small aw they are given by

$$
A=(\ell-s)(\ell+s+1)-a \omega \frac{2 m s^{2}}{\ell(\ell+1)}+\mathcal{O}\left(a^{2} \omega^{2}\right) .
$$

This expression provides a rational behind the analytic continuation $\ell \rightarrow \mathbb{R}$ : the angular eigenvalues are actually always non-integer for non-zero frequencies [59, 69, 70].

The purely incoming boundary condition for $R$ at the black hole horizon has the following form in the Boyer-Lindquist coordinates [49, 69]

$$
R=\text { const } \times\left(r-r_{+}\right)^{i Q-s}, \quad \text { as } r \rightarrow r_{+},
$$

where we have introduced

$$
Q \equiv \gamma m-\frac{2 M r_{+}}{r_{+}-r_{-}} \omega=\frac{a m-2 M r_{+} \omega}{r_{+}-r_{-}} .
$$

The solution of the Teukolsky equation at finite frequency that satisfies the purely incoming boundary condition at the horizon can be obtained in the form of a series over hypergeometric functions, see refs. [71-74],

$$
\begin{aligned}
R= & e^{-i \frac{\epsilon x}{\sqrt{1-\chi^{2}}}} x^{-s-i \epsilon / 2-i \tilde{Q}}(1+x)^{i \epsilon / 2+i \tilde{Q}}\left(\sum_{n=-\infty}^{\infty} a_{n}^{\nu} \frac{\Gamma(1-s+2 i Q) \Gamma(2 n+2 \nu+1)}{\Gamma(n+\nu+1+2 i \tilde{Q}) \Gamma(n+\nu+1-s-i \epsilon)}\right. \\
& \times x^{\nu+n}{ }_{2} F_{1}\left(-n-\nu+2 i \tilde{Q},-n-\nu+s+i \epsilon,-2 n-2 \nu,-x^{-1}\right) \\
& +\sum_{n=-\infty}^{\infty} a_{n}^{-\nu-1} \frac{\Gamma(1-s+2 i Q) \Gamma(2 n-2 \nu-1)}{\Gamma(n-\nu+2 i \tilde{Q}) \Gamma(n-\nu-s-i \epsilon)} \\
& \left.\times x^{-\nu-1+n}{ }_{2} F_{1}\left(-n+\nu+1+2 i \tilde{Q},-n+\nu+1+s+i \epsilon, 2 \nu+2-2 n,-x^{-1}\right)\right)
\end{aligned}
$$


where we have used $Q=\tilde{Q}-\epsilon / 2$ along with

$$
\begin{aligned}
\epsilon & \equiv r_{s} \omega, \quad \tilde{Q}=\frac{m \chi-r_{s} \omega}{2 \sqrt{1-\chi^{2}}}, \quad \nu=\ell+\Delta \ell \\
\Delta \ell & =\frac{\epsilon^{2}}{2 \ell+1}\left[-2-\frac{s^{2}}{\ell(\ell+1)}+\frac{\left((\ell+1)^{2}-s^{2}\right)^{2}}{(2 \ell+1)(2 \ell+2)(2 \ell+3)}-\frac{\left(\ell^{2}-s^{2}\right)^{2}}{(2 \ell-1) 2 \ell(2 \ell+1)}\right]+\mathcal{O}\left(\epsilon^{3}\right),
\end{aligned}
$$

The quantity $\nu$ is called "renormalized angular momentum." The coefficients $a_{n}^{\nu}$ and $a_{n}^{-\nu-1}$ satisfy certain recursion relations that are given in refs. [71-74]. In general they depend on $\epsilon$ parametrically and they are suppressed in the low-frequency limit, e.g. $a_{n}^{\nu}=\mathcal{O}\left(\epsilon^{|n|}\right)$ for $n \geq-\ell$. In what follows we will extract the part of the solution that has the desired source and response asymptotics at large distances. We will work at linear order in $\epsilon=r_{s} \omega$, in which case we will need only the following coefficients,

$$
\begin{aligned}
a_{0}^{-\nu-1} & =a_{0}^{\nu}=1, \quad a_{-1}^{\nu}=a_{1}^{-\nu-1}=i \epsilon \frac{(\ell+s)^{2}(\ell-2 i \tilde{Q})}{2 \ell^{2}(2 \ell+1)} \sqrt{1-\chi^{2}}+\mathcal{O}\left(\epsilon^{2}\right), \\
a_{1}^{\nu} & =a_{-1}^{-\nu-1}=i \epsilon \frac{(\ell-s+1)^{2}(\ell+1+2 i \tilde{Q})}{2(\ell+1)^{2}(2 \ell+1)} \sqrt{1-\chi^{2}}+\mathcal{O}\left(\epsilon^{2}\right) .
\end{aligned}
$$

The relevant solution that scales as $r^{\nu-s}\left(1+\mathcal{O}\left(r^{-2 \nu-1}\right)\right)$ at large distances in the smallfrequency limit is given by:

$$
\begin{aligned}
\left.R\right|_{r \rightarrow \infty} \supset & \text { const } \times e^{-i \frac{\omega r}{1-\chi^{2}}} r^{\nu-s}\left(1+\varkappa_{\nu m}^{(s)}\left(\frac{r_{s a}}{r}\right)^{2 \ell+1}\right)\left[\frac{\Gamma(2 \nu+1)}{\Gamma(\nu+1+2 i \tilde{Q}) \Gamma(\nu+1-s-i \epsilon)}\right. \\
& -a_{1}^{\nu} \frac{\Gamma(2 \nu+3)(-1-\nu+2 i \tilde{Q})(-1-\nu+s+i \epsilon)}{\Gamma(\nu+2+2 i \tilde{Q}) \Gamma(\nu+2-s-i \epsilon)(-2 \nu-2)} \\
& \left.-a_{1}^{\nu}(\nu+1) \frac{r_{+}}{r_{s a}} \frac{\Gamma(2 \nu+3)}{\Gamma(\nu+2+2 i \tilde{Q}) \Gamma(\nu+2-s-i \epsilon)}\right]+\mathcal{O}\left(\epsilon^{2}\right)
\end{aligned}
$$

where we have used the frequency-dependent response coefficient $k_{\nu m}^{(s)} \equiv \varkappa_{\nu m}^{(s)}\left(r_{s a} / r_{s}\right)^{2 \ell+1}$,

$$
\begin{aligned}
\varkappa_{\nu m}^{(s)} \equiv & \frac{\Gamma(\nu-s+1-i \epsilon) \Gamma(\nu+2 i \tilde{Q}+1) \Gamma(-2 \nu-1)}{\Gamma(2 \nu+1) \Gamma(-\nu-s-i \epsilon) \Gamma(2 i \tilde{Q}-\nu)} \\
& \times\left(1-a_{1}^{-\nu-1} \frac{(\nu+2 i \tilde{Q})(2 \nu+1)}{(\nu-2 i \tilde{Q})}-a_{1}^{\nu} \frac{(2 \nu+1)(1+\nu-2 i \tilde{Q})}{(\nu+1+2 i \tilde{Q})}\right. \\
& \left.+a_{1}^{\nu} \frac{r_{+}}{r_{s a}} \frac{2(\nu+1)^{2}(2 \nu+1)}{(\nu+1+2 i \tilde{Q})(\nu+1-s)}+a_{1}^{-\nu-1} \frac{r_{+}}{r_{s a}} \frac{2 \nu^{2}(2 \nu+1)}{(\nu-2 i \tilde{Q})(\nu+s)}\right)+\mathcal{O}\left(\epsilon^{2}\right) .
\end{aligned}
$$

For $\omega=0$ the response coefficient that appears in eq. (6.15) reduces to eq. (6.5). We will expand now this coefficient to linear order in $\epsilon$, while retaining black hole's spin to all orders. The $\epsilon \rightarrow 0$ limit of eq. (6.15) is complicated by the presence of a pole in the gamma functions. The ambiguity associated with this pole can be eliminated if we formally consider $\Delta \ell$ and $\epsilon$ as independent parameters, and use the expression eq. (6.12) only after 
regularizing the singularity. The presence of the pole also generates a finite logarithmic contribution, see appendix D. We obtain

$$
\begin{aligned}
\varkappa_{\nu m}^{(s), \text { finite }}= & {\left[\frac{i \gamma m}{\sinh (2 \pi \gamma m)} \sinh \left\{2 \pi \frac{r_{s} r_{+}}{r_{s a}}(\omega-m \Omega)\right\}-2\left(r_{s} \omega\right) \gamma m \ln x\right.} \\
& \left.-\frac{m a \omega(2 \ell+1)}{2(\ell+1) \ell}\left(\ell^{2}+\ell+s^{2}+2 i m \gamma\left(2 s+\frac{s^{2}}{\ell(\ell+1)}\right)\right)+(2 \ell+1) \gamma m r_{+} \omega\right] \\
& \times(-1)^{s} \frac{(\ell+s) !(\ell-s) !}{(2 \ell) !(2 \ell+1) !} \prod_{n=1}^{\ell}\left(n^{2}+4(\gamma m)^{2}\right)+\mathcal{O}\left(\left(r_{s} \omega\right)^{2},\left(r_{s} \omega\right)^{2} \ln x\right),
\end{aligned}
$$

where $\Omega$ is the black hole's angular velocity $\Omega \equiv a /\left(r_{+}^{2}+a^{2}\right)=a /\left(2 M r_{+}\right)$, and it is useful to recall that $x=\left(r-r_{+}\right) / r_{s a}$. The first important observation is that if we expand the response coefficient at leading order in black hole's spin and frequency of the external perturbation, we will find that eq. (6.16) matches the Newtonian expression (2.10) with the vanishing static Love number $\lambda_{\ell}=0$, but a non-zero dissipative part,

$$
k_{\nu m}^{(s)}=i r_{s}(\omega-m \Omega)(-1)^{s} \frac{(\ell+s) !(\ell-s) !(\ell !)^{2}}{(2 \ell) !(2 \ell+1) !}+\mathcal{O}\left(\omega \Omega, \omega^{2}, \Omega^{2}\right) .
$$

The dissipative imaginary response part vanishes for the locking frequency $\omega=m \Omega$. Therefore, at leading order in black hole's spin and frequency of the external perturbation the Kerr black holes behave like rigidly rotating dissipative spheres.

The second important observation is that generically the conservative response coefficients $\mathcal{O}(m \Omega)$ is not zero. Indeed, at face value, eq. (6.16) implies that the following time-dependent worldline operator does not vanish

$$
\int d \tau \mathcal{E}^{L} \dot{\mathcal{E}}^{L^{\prime}} \Lambda_{L L^{\prime}}^{(\omega \Omega)}
$$

where the spin-dependent coefficient $\Lambda_{L L^{\prime}}^{(\omega \Omega)}$ is odd w.r.t. time reversal. The third important observation is that for non-zero $\omega$ the tidal response coefficients exhibit classical renormalization-group running. This means that only the logarithmic part of the conservative frequency-dependent Love number appearing in eq. (6.16) is universal and independent of the renormalization scheme. This situation can be contrasted with the $\omega=0$ Love numbers, which do not receive any logarithmic contributions and hence do not run with distance $[6,75] .{ }^{17}$ Fourth, eq. (6.11) can be used to extract the Love numbers that depend on the frequency squared. They will be interesting to compare with recent results on frequency-dependent Love numbers given in ref. [28]. We leave this question for future work.

Finally, let us comment on the near-field approximation, which has been recently used to compute Love numbers from the Teukolsky equation [26]. We present this calculation for a generic spin $s$ perturbation in appendix D. There we show that the near-field expansion does not exactly map onto the small frequency expansion. As a result, the leading order near-field approximation does not fully capture the $\mathcal{O}(\omega \Omega)$ corrections to the tidal response coefficients.

\footnotetext{
${ }^{17}$ This is true in four dimensions. In certain spacetime dimensions the static Love numbers also exhibit renormalization-group running $[7,11]$.
} 


\section{Discussion and conclusions}

In this work we have computed the static response of Kerr black holes to external electromagnetic and scalar perturbations in four dimensions. This complements the analysis of refs. [25, 26], which have calculated the response of Kerr black holes to spin-2 (gravitational) perturbations. Our main results are summarized in the master formula (6.5), which displays the Kerr black hole static response coefficients for a perturbing field with generic integer spin $s$. Importantly, all responses are purely dissipative, i.e. the Love numbers for spinning black holes identically vanish for spin-0, spin-1, and spin-2 fields to all orders in black hole spin. We have also extended our results to leading frequency-dependent effects, which also include the running of response coefficients, see eq. (6.16).

We have used the gauge-invariant definition of Love numbers as Wilson coefficients in the point-particle effective field theory (EFT). To that end we have introduced local finitesize operators in the EFT and have extracted the relevant Wilson coefficients by matching the EFT and full GR calculations. We have also shown that the EFT allows one to clearly separate dissipative and conservative responses. The key ingredient of our matching procedure is the analytic continuation of relevant static response solutions to non-integer values of the orbital multipole number $\ell$. We have explicatively shown that this procedure allows one to extract the response coefficients from full general relativity solutions in a coordinateindependent fashion. Moreover, we have interpreted this procedure in the EFT context and have shown that it helps to separate non-linear gravity corrections to perturbing sources (i.e. source-graviton EFT diagrams) from corrections generated by the induced multipole moments. Curiously, we have found that the subleading source corrections exactly cancel the response part in the advanced Kerr coordinates. It will be interesting to understand the origin of this cancellation in the future.

We have demonstrated that spinning black holes are very similar to the static ones from the EFT point of view: both of them can be described with a single point-particle term in the worldline action in the static limit. Our analysis suggests that the case of spinning black holes may be useful to understand the vanishing of tidal Love numbers in four dimensions and a possible EFT naturalness problem related to that. In particular, we have shown that this problem may be addressed at the level of the massless scalar field. This is a very simplistic model, yet it captures many qualitative details of Love number calculations relevant for both static and spinning black holes. This suggests that the scalar field toy model may play an important role in elucidating the nature of vanishing of local finite-size EFT operators for black holes.

Our analysis can be extended in multiple ways. First, one can compute Love numbers and the relevant Wilson coefficients of the point-particle EFT for spinning black holes in spacetime dimensions greater than four. The properties of higher dimensional spinning black holes are known to differ significantly from their four dimensional counterparts (see e.g. $[76,77])$, and hence we can expect interesting consequences for response coefficients there. Second, one can study the relationship between dissipative spin- 0 and spin- 1 response coefficients that we have computed and the phenomenon of black hole torques along the lines of $[25,27,53]$. Third, it would be interesting to compute the Love coefficients for 
charged spinning Kerr-Newman black holes [78]. Fourth, one can carry out a systematic analysis of the frequency-dependent Love numbers for spinning black holes. Eventually, it will be important to understand if there is an extra symmetry of the Schwarzschild and Kerr spacetimes which makes the conservative static black hole response vanish in four dimensions. We leave these research directions for future work.

Note added. While this paper was being prepared, refs. [26-28] appeared. These papers have some overlap with our work in the interpretation of dissipative response coefficients. In particular, we have independently obtained that the response coefficients presented as "Love numbers" in ref. [25] actually correspond to purely non-conservative effects.

\section{Acknowledgments}

This work is supported in part by the NSF award PHY-1915219 and by the BSF grant 2018068. MI is partially supported by the Simons Foundation's Origins of the Universe program.

\section{A Useful mathematical relations}

\section{A.1 Spherical harmonics}

Scalar Spherical Harmonics. We use the following definition for the (scalar) spherical harmonics

$$
Y_{\ell m}(\theta, \phi)=\frac{(-1)^{\ell+\frac{|m|+m}{2}}}{2^{\ell} \ell !}\left[\frac{2 \ell+1}{4 \pi} \frac{(\ell-|m|) !}{(\ell+|m|) !}\right]^{1 / 2} e^{i m \phi}(\sin \theta)^{|m|}\left(\frac{d}{d \cos \theta}\right)^{\ell+|m|}(\sin \theta)^{2 \ell},
$$

valid for $\ell \geq 0,-\ell<m<\ell$. These harmonics obey the following relations

$$
\Delta_{\mathbb{S}^{2}} Y_{\ell m}=-\ell(\ell+1) Y_{\ell m}, \quad Y_{\ell m}^{*}(\mathbf{n})=(-1)^{m} Y_{\ell(-m)}(\mathbf{n}), \quad \oint_{\mathbb{S}^{2}} d \Omega Y_{\ell m} Y_{\ell^{\prime} m^{\prime}}^{*}=\delta_{\ell \ell^{\prime}} \delta_{m m^{\prime}},
$$

where $\Delta_{\mathbb{S}^{2}}$ is the two-sphere Laplacian.

Spin-Weighted Spherical Harmonics. One can introduce the following spin $s$-raising and spin $s$-lowering operators,

$$
\partial \equiv-\left(\partial_{\theta}+\frac{i}{\sin \theta} \partial_{\phi}-s \frac{\cos \theta}{\sin \theta}\right), \quad \bar{\partial} \equiv-\left(\partial_{\theta}-\frac{i}{\sin \theta} \partial_{\phi}+s \frac{\cos \theta}{\sin \theta}\right) .
$$

Applying these operators on the (scalar) spherical harmonics $Y_{\ell m} \equiv{ }_{0} Y_{\ell m}$ one can define the spin-weighted spherical harmonics for $\ell \geq|s|$,

$$
\begin{aligned}
& \partial\left({ }_{s} Y_{\ell m}\right)=+\sqrt{(\ell-s)(\ell+s+1)_{s+1}} Y_{\ell m}, \\
& \bar{\partial}\left({ }_{s} Y_{\ell m}\right)=-\sqrt{(\ell+s)(\ell-s+1)_{s-1}} Y_{\ell m} .
\end{aligned}
$$

These harmonics obey the following relations

$$
{ }_{s} Y_{\ell m}^{*}(\mathbf{n})=(-1)^{m+s}{ }_{-s} Y_{\ell(-m)}(\mathbf{n}), \quad \oint_{\mathbb{S}^{2}} d \Omega{ }_{s} Y_{\ell m s} Y_{\ell^{\prime} m^{\prime}}^{*}=\delta_{\ell \ell^{\prime}} \delta_{m m^{\prime}} .
$$


Transverse Vector Spherical Harmonics. The transverse vector analog of scalar spherical harmonics are defined as follows

$$
\vec{Y}_{\ell m}^{T} \equiv-\frac{1}{\sqrt{\ell(\ell+1)}} \vec{r} \times \vec{\nabla} Y_{\ell m}, \quad Y_{i \ell m}^{T}=-\frac{\sqrt{\operatorname{det} g_{3}} \varepsilon_{i j k}}{\sqrt{\ell(\ell+1)}} x^{j} \nabla^{k} Y_{\ell m}
$$

where $g_{3}$ is the $3 \mathrm{~d}$ metric, $\varepsilon_{i j k}$ is the three-dimensional Levi-Civita symbol $\left(\varepsilon_{123}=1\right)$ and $\nabla^{k}$ is the corresponding covariant derivative. Note that there is a sign difference between our definition and the one adopted in ref. [41]. We also stress that $\varepsilon_{i j k}$ denotes the fully-antisymmetric symbol, whereas $\epsilon_{i j k}$ stands for the anti-symmetric tensor,

$$
\epsilon_{i j k}=\sqrt{g_{3}} \varepsilon_{i j k}, \quad \epsilon^{i j k}=\frac{\varepsilon^{i j k}}{\sqrt{g_{3}}} .
$$

The transverse spin-1 spherical harmonics are related to the spin-weighted spherical harmonics through

$$
Y_{i \ell m}^{T}=\frac{i}{\sqrt{2}}\left({ }_{-1} Y_{\ell m} m^{i}+{ }_{+1} Y_{\ell m} m^{* i}\right) .
$$

Since $\vec{Y}_{\ell m}^{T}$ are orthogonal to the radial direction $n_{i}$, it is also convenient to use their projections onto $\mathbb{S}^{2}$, known as the Regge-Wheeler (RW) vector spherical harmonics [63, 79]

$$
Y_{a}^{\mathrm{RW}}{ }_{\ell m} \equiv \frac{1}{\sqrt{\ell(\ell+1)}} \sqrt{g_{2}} \varepsilon_{a b} g_{2}^{b c} \nabla_{c} Y_{\ell m}
$$

where $a=(\theta, \phi), g_{2}^{a b}$ is the metric tensor on $\mathbb{S}^{2}, g_{2} \equiv \operatorname{det} g_{2}, \nabla_{a}$ is the covariant derivative on $\mathbb{S}^{2}$, and we have introduced the flat-space 2-dimensional Levi-Civita symbol

$$
\varepsilon_{\theta \phi}=-\varepsilon_{\phi \theta}=1, \quad \varepsilon_{\theta \theta}=\varepsilon_{\phi \phi}=0 .
$$

In our conventions the RW and the vector harmonics defined in eq. (A.6) coincide in the orthonormal spherical coordinates basis. The $2 \mathrm{~d}$ transverse spherical harmonics satisfy [7, 79]:

$$
\Delta_{\mathbb{S}^{2}} Y_{a \ell m}^{\mathrm{RW}}=-(\ell(\ell+1)-1) Y_{a \ell m}^{\mathrm{RW}}, \quad \oint_{\mathbb{S}^{2}} d \Omega g_{2}^{a b} Y_{a \ell m}^{\mathrm{RW}} Y_{b \ell^{\prime} m^{\prime}}^{\mathrm{RW} *}=\delta_{\ell \ell^{\prime}} \delta_{m m^{\prime}} .
$$

Symmetric Trace-Free Tensors. Finally, instead of the spherical harmonics it may be conveneient to use the basis of the symmetric trace-free tensors of rank $\ell$ ("STF- $\ell$ tensors") [41]. These tensors generate an irreducible representation of $\mathrm{SO}(3)$ and hence there exists a one-to-one mapping between them and the spherical harmoncis. This mapping is realized via

$$
Y_{\ell m}=\mathscr{Y}_{\ell m}^{* L} n_{\langle L\rangle}, \quad \text { or } \quad n^{\langle L\rangle}=\frac{4 \pi \ell !}{(2 \ell+1) ! !} \sum_{m=-\ell}^{\ell} Y_{\ell m} \mathscr{Y}_{\ell m}^{L}
$$

where the constant STF tensors $\mathscr{Y}_{\ell m}^{L}$ satisfy

$$
\mathscr{Y}_{\ell m}^{L}=\frac{(2 \ell+1) ! !}{4 \pi \ell !} \oint_{\mathbb{S}^{2}} d \Omega n_{\langle L\rangle} Y_{\ell m}^{*}, \quad \mathscr{Y}_{\ell(-m)}^{L}=(-1)^{m} \mathscr{Y}_{\ell m}^{* L} .
$$


Since $\mathscr{Y}_{\ell m}^{L}$ tensors form a basis for the $(2 \ell+1)$ dimensional vector space of the STF tensors on $\mathbb{S}^{2}$, any STF tensor can be expanded over them as

$$
\mathcal{F}^{L}=\sum_{m=-\ell}^{\ell} \mathscr{Y}_{\ell m}^{* L} \mathcal{F}_{\ell m}, \quad \mathcal{F}_{\ell m}=\frac{4 \pi \ell !}{(2 \ell+1) ! !} \mathscr{Y}_{\ell m}^{L} \mathcal{F}_{L} .
$$

All in all, any scalar function on $\mathbb{S}^{2}$ can be represented as

$$
F(\theta, \phi)=\sum_{\ell=0}^{\infty} \sum_{m=-\ell}^{\ell} \mathcal{F}_{\ell m} Y_{\ell m}=\sum_{\ell=0}^{\infty} \mathcal{F}^{L} n_{\langle L\rangle} .
$$

Some other important identities are

$$
\begin{gathered}
\varepsilon_{j p q} \mathscr{Y}_{p(L-1)}^{* \ell m} \mathscr{Y}_{q(L-1)}^{\ell m}=-i m \frac{(2 \ell+1) ! !}{4 \pi \ell ! \ell} \hat{\xi}_{j}^{0}, \\
\varepsilon_{j p q} \mathscr{Y}_{p(L-1)}^{* \ell m} \mathscr{Y}_{q(L-1)}^{\ell(m+1)}=-i \frac{(2 \ell+1) ! !}{4 \pi \ell ! 2 \ell}[2(\ell-m)(\ell+m+1)]^{1 / 2} \hat{\xi}_{j}^{-1}, \\
\varepsilon_{j p q} \mathscr{Y}_{p(L-1)}^{* \ell m} \mathscr{Y}_{q(L-1)}^{\ell(m-1)}=i \frac{(2 \ell+1) ! !}{4 \pi \ell ! 2 \ell}[2(\ell+m)(\ell-m+1)]^{1 / 2} \hat{\xi}_{j}^{+1}, \\
\varepsilon_{j p q} \mathscr{Y}_{p(L-1)}^{* \ell m} \mathscr{Y}_{q(L-1)}^{\ell(m+\mu)}=0 \text { if } \mu \neq 0 \text { or } \pm 1,
\end{gathered}
$$

where $\hat{\xi}_{j}^{0}=\delta_{j}^{3}, \hat{\xi}_{j}^{ \pm 1}=\mp\left(\delta_{j}^{1} \pm i \delta_{j}^{2}\right) / \sqrt{2}$.

\section{A.2 Gamma function}

The Euler Gamma funciton is defined via

$$
\Gamma(x+1)=\Gamma(x) x .
$$

We use several important relations in the main text

$$
|\Gamma(1+\ell+b i)|^{2}=\frac{\pi b}{\sinh (\pi b)} \prod_{n=1}^{\ell}\left(n^{2}+b^{2}\right) \quad \text { for } \quad \ell \in \mathbb{N},
$$

as well as $\Gamma\left(z^{*}\right)=\Gamma^{*}(z)$, and Euler's reflection formula,

$$
\Gamma(z) \Gamma(1-z)=\frac{\pi}{\sin (\pi z)} .
$$

We also need the Taylor expansions of the Gamma function around its poles that correspond to natural values of the orbital number $\ell$. To obtain them, we shift the argument of the Gamma function as $\ell \rightarrow \ell+\varepsilon, \varepsilon \ll 1$, which yields

$$
\frac{1}{\Gamma(2 \ell+1) \Gamma(-2 \ell)}=-2 \varepsilon, \quad \frac{1}{\Gamma(-\ell)}=(-1)^{\ell+1}(\ell !) \varepsilon .
$$

These expressions lead to the following relations

$$
\begin{array}{llrl}
\frac{\Gamma(-2 \ell-1)}{\Gamma(-\ell)} & =\frac{(-1)^{\ell+1} \ell !}{2(2 \ell+1) !}, & \frac{\Gamma(-2 \ell-1)}{\Gamma(-\ell-2)} & =\frac{(-1)^{\ell+1}(\ell+2) !}{2(2 \ell+1) !}, \\
\frac{\Gamma(-2 \ell-1)}{\Gamma(-\ell-1)} & =\frac{(-1)^{\ell}(\ell+1) !}{2(2 \ell+1) !}, & \frac{\Gamma(-2 \ell)}{\Gamma(-\ell)} & =\frac{(-1)^{\ell} \ell !}{2(2 \ell) !} .
\end{array}
$$




\section{A.3 Gauss hypergeometric function}

The classic hypergeometric equation has the following form

$$
x(1-x) y^{\prime \prime}+(c-(1+a+b) x) y^{\prime}-a b y=0 .
$$

If $c \neq 0,-1,-2, \ldots$, this equation has the following solution in terms of the Gauss hypergeometric function

$$
y={ }_{2} F_{1}(a, b, c, x) \equiv \sum_{n=0}^{\infty} \frac{\Gamma(n+a) \Gamma(n+b)}{\Gamma(a) \Gamma(b)} \frac{\Gamma(c)}{\Gamma(n+c)} \frac{x^{n}}{n !} .
$$

The other indepedent solution of eq. (A.22) is given by

$$
y=x^{1-c} F_{1}(b-c+1, a-c+1,2-c, x) .
$$

This solution is singular at $z=0$.

If $c=-n$, where $n=0,1,2, \ldots$, the (regular at $x=0$ ) solution to eq. (A.22) takes the following form

$$
y=x^{1+n} F_{1}(a+n+1, b+n+1, n+2, x) .
$$

If $a=-n, n=0,1,2, \ldots$ and $c=-m, m=n, n+1, n+2, \ldots$ the hypergeometric series truncates at order $m$. If $a+b-c<0$, the hypergeometric series converges at $|x|=1$. Otherwise it generically converges for $|x|<1$ (unless it is a polynomial).

The hypergeometric function (A.23) can be analytically continued at $x=\infty$ via

$$
\begin{aligned}
{ }_{2} F_{1}(a, b, c, x)= & \frac{\Gamma(c) \Gamma(b-a)}{\Gamma(b) \Gamma(c-a)}(-x)_{2}^{-a} F_{1}\left(a, a+1-c, a+1-b, x^{-1}\right) \\
& +\frac{\Gamma(c) \Gamma(a-b)}{\Gamma(a) \Gamma(c-b)}(-x)^{-b}{ }_{2} F_{1}\left(b, b+1-c, b+1-a, x^{-1}\right),
\end{aligned}
$$

and around $x=1$ via

$$
\begin{aligned}
{ }_{2} F_{1}(a, b, c, x)= & \frac{\Gamma(c) \Gamma(c-a-b)}{\Gamma(c-a) \Gamma(c-b)}{ }_{2} F_{1}(a, b, a+b+1-c, 1-x) \\
& +\frac{\Gamma(c) \Gamma(-c+a+b)}{\Gamma(a) \Gamma(b)}(1-x)^{c-a-b}{ }_{2} F_{1}(c-a, c-b, c+1-a-b, 1-x) .
\end{aligned}
$$

\section{B Calculation of Maxwell-Newman-Penrose scalars}

In this appendix we compute the stationary electromagnetic field around the Kerr black hole for a source located at spatial infinity. This is the calculation relevant for the extraction of the response coefficients. In the Newman-Penrose formalism, the electromagnetic tensor $F_{\mu \nu}$ is represented in terms of 3 complex scalar functions,

$$
\Phi_{0}=F_{\mu \nu} l^{\mu} m^{\nu}, \quad \Phi_{1}=\frac{1}{2} F_{\mu \nu}\left(l^{\mu} n^{\nu}+m^{* \mu} m^{\nu}\right), \quad \Phi_{2}=F_{\mu \nu} m^{* \mu} n^{\nu},
$$

where $l^{\mu}, n^{\mu}, m^{\mu}$ are the NP null tetrades and $m^{*}$ is the complex conjugate of $m^{\mu}$. In what follows we will use the Boyer-Lindquist coordinates, in which the Kinnersley tetrades are 
given by eq. (4.11). Instead of the usual scalars $\Phi_{0}, \Phi_{1}, \Phi_{2}$, it is convenient to work in terms of the rescaled scalars,

$$
\tilde{\Phi}_{0}=\Phi_{0}, \quad \tilde{\Phi}_{1}=\frac{(r-i a \cos \theta)^{2}}{\left(r_{+}-r_{-}\right)^{2}} \Phi_{1}, \quad \tilde{\Phi}_{2}=\frac{(r-i a \cos \theta)^{2}}{\left(r_{+}-r_{-}\right)^{2}} \Phi_{2} .
$$

The stationary (i.e. $\omega=0$ ) vacuum Maxwell equations take the following form in terms of the NP quantities $[48,49]$ :

$$
\begin{array}{r}
\sqrt{2} r_{s a}^{2}\left(\partial_{r}+\frac{a}{\Delta} \partial_{\phi}\right) \tilde{\Phi}_{1}-(r-i a \cos \theta)\left(\partial_{\theta}+\cot \theta-\frac{i}{\sin \theta} \partial_{\phi}\right) \tilde{\Phi}_{0}+i a \sin \theta \tilde{\Phi}_{0}=0 \\
\sqrt{2} r_{s a}^{2}\left(\partial_{\theta}+\frac{i}{\sin \theta} \partial_{\phi}\right) \tilde{\Phi}_{1}+(r-i a \cos \theta)\left(\partial_{r}-\frac{a}{\Delta} \partial_{\phi}\right) \Delta \tilde{\Phi}_{0}-\Delta \tilde{\Phi}_{0}=0 \\
\frac{1}{\sqrt{2}}\left(\partial_{\theta}-\frac{i}{\sin \theta} \partial_{\phi}\right) \tilde{\Phi}_{1}-(r-i a \cos \theta)\left(\partial_{r}+\frac{a}{\Delta} \partial_{\phi}\right) \tilde{\Phi}_{2}+\tilde{\Phi}_{2}=0 \\
\frac{1}{\sqrt{2}}\left(\partial_{r}-\frac{a}{\Delta} \partial_{\phi}\right) \tilde{\Phi}_{1}+(r-i a \cos \theta)\left(\partial_{\theta}+\cot \theta+\frac{i}{\sin \theta} \partial_{\phi}\right) \frac{\tilde{\Phi}_{2}}{\Delta}-\frac{i a \sin \theta}{\Delta} \tilde{\Phi}_{2}=0
\end{array}
$$

Teukolsky has shown that $\tilde{\Phi}_{0}$ and $\tilde{\Phi}_{2}$ factorize in the Kerr background as

$$
\tilde{\Phi}_{2}=\sum_{\ell m} a_{\ell m} R_{\ell m}^{(2)}(r){ }_{-1} Y_{\ell m}(\theta, \phi), \quad \tilde{\Phi}_{0}=\sum_{\ell m} a_{\ell m} R_{\ell m}^{(0)}(r)+1 Y_{\ell m}(\theta, \phi),
$$

where the radial harmonic $R_{\ell m}^{(2)}$ satisfies eq. (4.16). Applying the operator

$$
\partial_{\theta}-i \partial_{\phi} / \sin \theta
$$

to eq. (B.3a) and the operator

$$
\partial_{r}+a \partial_{\phi} / \Delta
$$

to eq. (B.3c), we find eq. (4.21), which means we have obtained both $\tilde{\Phi}_{0}$ and $\tilde{\Phi}_{2}$. It is important to express the radial function $R_{\ell m}^{(0)}$ as a second derivative over $R_{\ell m}^{(2)}$ because we will have to integrate over it to get $\tilde{\Phi}_{1}$.

The calculation of $\tilde{\Phi}_{1}$ is more intricate as it does not factorize in $\theta$ and $x$. The axial symmetry suggests the following ansatz

$$
\tilde{\Phi}_{1}=\sum_{m=-\infty}^{\infty}\left(\frac{x}{1+x}\right)^{-i \gamma m} e^{i m \phi} \tilde{\Phi}_{1 m}(x, \theta) .
$$

Plugging this into eq. (B.3a) and integrating over $x$ (which is related to the radial coordinate $r$ ) we obtain (4.22) plus an integration constant, which corresponds to black hole's charge. Since we consider the neutral black holes, we put this constant to zero.

\section{Spin-1 magnetic Love numbers}

Due to the presence of magnetic-electric duality in four dimensions, we have anticipated that the electric and magnetic response coefficients would coincide in the Kerr background. In this appendix we explicitly show it. To that end, we extract the magnetic response from 
the Maxwell-Newman-Penrose scalar $\Phi_{1}$ and match them to the Wilson coefficients of the magnetic field worldline EFT.

Our first goal is to extract the Newtonian response coefficients from the Kerr solution using eq. (4.8). We will match one particular component, $F_{\theta \phi}$. To proceed, we need to simplify the commutator $\nabla_{[a} Y_{b] \ell}^{\mathrm{RW}}$. A crucial observation is that ${ }^{18}$

$$
\begin{aligned}
\nabla_{[\theta} Y_{\phi]}^{\mathrm{RW}} \ell m & =\frac{1}{2 \sqrt{\ell(\ell+1)}}\left[\nabla_{\theta}\left(\sqrt{\operatorname{det} g_{2}} \varepsilon_{\phi \theta} g_{2}^{\theta \theta} \nabla_{\theta}\right)-\nabla_{\phi}\left(\sqrt{\operatorname{det} g_{2}} \varepsilon_{\theta \phi} g_{2}^{\phi \phi} \nabla_{\phi}\right)\right] Y_{\ell m} \\
& =\frac{1}{2 \sqrt{\ell(\ell+1)}}\left[-\sqrt{\operatorname{det} g_{2}} \Delta_{\mathbb{S}^{2}}\right] Y_{\ell m}=\frac{\sin \theta}{2} \sqrt{\ell(\ell+1)} Y_{\ell m} .
\end{aligned}
$$

Thus, we have

$$
F_{\theta \phi}=\sum_{\ell=1} \sum_{m=-\ell}^{\ell} \sin \theta Y_{\ell m} \beta_{\ell m} r^{1+\ell}\left[1-\frac{\ell+1}{\ell} \tilde{k}_{\ell m}^{(1)}\left(\frac{r}{r_{s}}\right)^{-2 \ell-1}\right] .
$$

To extract the magnetic Love numbers, we need to compare this expression with our formula for $F_{\theta \phi}$ that we have obtained by solving for $\Phi_{1}$. We have

$$
\begin{aligned}
\left.F_{\theta \phi}\right|_{r \rightarrow \infty} & =2 \operatorname{Im} \Phi_{1} r^{2} \sin \theta \\
& =\left.2 r^{2} \sin \theta \frac{\sqrt{2} r_{s a}^{2}}{r^{2}} \operatorname{Im} \sum_{\ell=1} \sum_{m=-\ell}^{\ell} \frac{a_{\ell m} Y_{\ell m}}{(\ell(\ell+1))^{1 / 2}}\left[x \frac{d}{d x}\left(y_{\ell m}\right)-y_{\ell m}\right]\right|_{x=\left(r-r_{+}\right) / r_{s a}} \\
& =\sin \theta \sum_{\ell=1} \sum_{m=-\ell}^{\ell} \beta_{\ell m} Y_{\ell m} F_{\ell m}^{\theta \phi}(r)
\end{aligned}
$$

where in the last line we have used eq. (4.24) and we have also introduced the new function

$$
F_{\ell m}^{\theta \phi}=r_{s a}^{\ell+1} x^{\ell+1} \cdot\left[1-x^{-2 \ell-1} \frac{\ell+1}{\ell} \frac{\Gamma(-2 \ell-1) \Gamma(\ell+2) \Gamma(\ell+2 i m \gamma+1)}{\Gamma(1-\ell) \Gamma(2 \ell+1) \Gamma(2 i m \gamma-\ell)}\right] .
$$

Note that the sum $\sum_{\ell m} \beta_{\ell m} Y_{\ell m}\left(x y_{\ell m}^{\prime}-y_{\ell m}\right)$ is real.

Matching this with eq. (C.2) we obtain that the magnetic and electric response coefficients coincide in the Kerr background

$$
\tilde{k}_{\ell m}^{(1)}=k_{\ell m}^{(1)} .
$$

Matching to the EFT can be done using the EFT electromagnetic action eq. (4.1). In analogy with the electric field we introduce an external background magnetic field source as

$$
\begin{aligned}
\bar{A}_{j}=\frac{r^{\ell}}{\sqrt{\ell(\ell+1)}} \sum_{m=-\ell}^{\ell} \bar{\beta}_{\ell m} Y_{j \ell m}^{T} & =-\frac{\sqrt{g_{3}} \varepsilon_{j i k} x^{i} \nabla^{k}}{\ell(\ell+1)} \sum_{m=-\ell}^{\ell} r^{\ell} \bar{\beta}_{\ell m} Y_{\ell m} \\
& =-\frac{\sqrt{g_{3}} \varepsilon_{j i k} x^{i} \nabla^{k}}{\ell(\ell+1)} \bar{\beta}_{i_{1} \ldots i_{\ell}} x^{i_{1} \ldots i_{\ell}},
\end{aligned}
$$

\footnotetext{
${ }^{18}$ It can be shown that in general $2 \nabla_{\left[a \sqrt{g_{2}}\right.} \varepsilon_{b] c} \nabla^{c}=-\sqrt{g_{2}} \varepsilon_{a b} \nabla^{2}$.
} 
where $\bar{\beta}_{i_{1} \ldots i_{\ell}}$ is the STF tensor. By construction, our source $\bar{A}_{j}$ is manifestly harmonic $\nabla^{2} \bar{A}_{j}=0$ and transverse $\nabla^{j} \bar{A}_{j}=0$ and hence it satisfies the spatial part of the Maxwell equations $\nabla^{\mu} F_{\mu j}=0$. Expanding the Maxwell action to quadratic order in $A_{j}$ and solving perturbatively its equation of motion with the coupling to the source included, we obtain

$$
A_{j}=\sum_{m=-\ell}^{\ell} \bar{\beta}_{\ell m} r^{\ell} Y_{j \ell m}^{T}-\frac{\sqrt{g_{3}} \varepsilon_{j i^{\prime} k^{\prime}} x^{i^{\prime}} \nabla^{k^{\prime}}}{\ell(\ell+1)} \tilde{\lambda}_{\substack{(1) i_{1} \ldots i_{\ell} \\ i_{1}^{\prime} \ldots i_{\ell}}}^{\bar{\beta}_{i_{1} \ldots i_{\ell}}} n^{\left\langle i_{1}^{\prime} \ldots i_{\ell}^{\prime}\right\rangle}(-1)^{\ell} \frac{2^{\ell-2}}{\pi^{1 / 2} \Gamma(1 / 2-\ell)} r^{-\ell-1} .
$$

Now we are in position to match the angular component of the magnetic tensor $F_{\theta \phi}$. As a first step we compute the response part of the Maxwell tensor $F_{a b}^{\text {response }}$ from eq. (C.7) and use that $2 \sqrt{g_{3}} \varepsilon_{[b j k} x^{j} \nabla^{k} \nabla_{a]}=\sqrt{g_{2}} \varepsilon_{a b} r \nabla_{\mathbb{S}^{2}}^{2}$ in the spherical coordinate basis, which yields

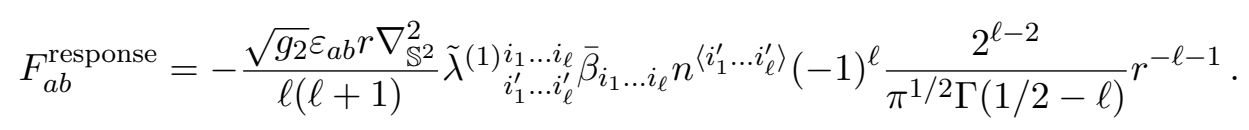

This can be compared with eq. (C.3), which we rewrite for a single orbital harmonic $\ell$ as follows:

$$
F_{\theta \phi}=-\frac{\sqrt{g_{2}} \varepsilon_{\theta \phi} r \nabla_{\mathbb{S}^{2}}^{2}}{\ell(\ell+1)} \sum_{m=-\ell}^{\ell} \beta_{\ell m} Y_{\ell m} F_{\ell m}^{\theta \phi}(r) / r .
$$

Now we can rewrite the expression above using the Thorne STF tensors and arrive at the anticipated result

$$
\tilde{\lambda}^{(1)^{i_{1}^{\prime} \ldots i_{\ell}^{\prime}}}=\frac{r_{s}^{2 \ell+1}}{B_{\ell} \ldots i_{\ell}} \frac{4 \pi \ell !}{(2 \ell+1) ! !} \frac{-(\ell+1)}{\ell} \sum_{m=-\ell}^{\ell} \tilde{k}_{\ell m}^{(1)} \mathscr{Y}_{\ell m}^{* i_{1} \ldots i_{\ell}} \mathscr{Y}_{\ell m}^{i_{1}^{\prime} \ldots i_{\ell}^{\prime}}
$$

where $B_{\ell}$ a constant is given in eq. (3.31). This expression coincides with the electric response coefficient tensor given in eq. (4.32) up to a factor $(\ell+1) / \ell$.

\section{Comment on the near-field approximation}

In this appendix we discuss the validity of the solution of the frequency-dependent radial Teukolsky equation (6.7) in the near-field approximation. We start with the Klein-Gordon equation in the Schwarzschild background.

\section{D.1 Scalar field example}

The differential equation defining the radial mode function of the scalar field in the Schwarzschild background takes the following form $[59,69]$

$$
x(1+x) R^{\prime \prime}+(2 x+1) R^{\prime}+\left[\left(r_{s} \omega\right)^{2} \frac{(1+x)^{4}}{x(1+x)}-\ell(\ell+1)\right] R=0 .
$$

The near field approximation amounts to replacing $[59,69,70]$

$$
\frac{\left(r_{s} \omega\right)^{2}(1+x)^{4}}{x(1+x)} \rightarrow \frac{\left(r_{s} \omega\right)^{2}}{x(1+x)}
$$


The corrections to the r.h.s. term are small as long as

$$
\left(r_{s} \omega\right) x \ll(\ell+1) .
$$

The near-zone approximation can be systematically formulated by an introduction of a formal expansion parameter $\alpha$ such that

$$
(1+x)^{4} \rightarrow(1+\alpha x)^{4}=1+\mathcal{O}(\alpha x)
$$

The final expressions have to be evaluated at $\alpha=1$. Then the corrections beyond eq. (D.2) can be systematically computed order-by-order in $\alpha$. However, we note that this does not correspond to a low-frequency expansion with the small parameter $r_{s} \omega \ll 1$. Indeed, sufficiently far from the horizon, i.e. for $x=\mathcal{O}(1)$, we have

$$
\left(r_{s} \omega\right)^{2} \sim\left(r_{s} \omega\right)^{2} x^{2}
$$

and hence keeping the term $\left(r_{s} \omega\right)^{2}$ while neglecting the term $\left(r_{s} \omega\right)^{2} x^{2}$ in eq. (D.2) is not justified by the smallness of $r_{s} \omega$.

Now let us write eq. (D.1) in the zeroth order near zone approximation $(\alpha x \ll 1)$,

$$
x(1+x) R^{\prime \prime}+(2 x+1) R^{\prime}+\left[\frac{\left(r_{s} \omega\right)^{2}}{x(1+x)}-\ell(\ell+1)\right] R=0 .
$$

The solution consistent with the purely incoming boundary condition at the horizon is given by

$$
R=\mathrm{const} \cdot\left(\frac{x}{1+x}\right)_{2}^{i \omega r_{s}}{ }_{2} F_{1}\left(\ell+1,-\ell, 1+2 i r_{s} \omega,-x\right)
$$

If we now analytically continue this solution for $x \gg 1$, we will obtain,

$$
\begin{aligned}
& R= \text { const } \cdot\left(\frac{x}{1+x}\right)^{i \omega r_{s}}\left(\frac{\Gamma\left(1+2 i r_{s} \omega\right) \Gamma(2 \ell+1)}{\Gamma(\ell+1) \Gamma\left(1+\ell+2 i r_{s} \omega\right)} x^{\ell}{ }_{2} F_{1}\left(-\ell,-\ell-2 i r_{s} \omega,-2 \ell,-x^{-1}\right)\right. \\
&\left.+\frac{\Gamma\left(1+2 i r_{s} \omega\right) \Gamma(-2 \ell-1)}{\Gamma(-\ell) \Gamma\left(-\ell+2 i r_{s} \omega\right)} x^{-\ell-1} \cdot{ }_{2} F_{1}\left(\ell+1, \ell+1-2 i r_{s} \omega, 2 \ell+2,-x^{-1}\right)\right) \\
& \underset{x \rightarrow \infty}{\longrightarrow} r_{s}^{\ell} x^{\ell}\left(1+k_{\ell}^{(0) \mathrm{NF}} x^{-2 \ell-1}\right)
\end{aligned}
$$

where the coefficient $k_{\ell}^{(0) \mathrm{NF}}$ might be interpreted as a frequency-dependent response coefficient,

$$
k_{\ell}^{(0) \mathrm{NF}} \equiv \frac{\Gamma(-2 \ell-1) \Gamma(\ell+1) \Gamma\left(1+\ell+2 i r_{s} \omega\right)}{\Gamma(2 \ell+1) \Gamma(-\ell) \Gamma\left(-\ell+2 i r_{s} \omega\right)} .
$$

Indeed, for $\omega=0$ this expression reproduces the scalar Love number for the Schwarzschild black hole. However, strictly speaking, we cannot use eq. (D.9) for the Love number matching because there may be other frequency-dependent contributions that have been omitted in the near zone approximation. This will be shown shortly when we compare eq. (D.9) with the accurate solution to the Teukolsky equation. 


\section{D.2 Teukolsky equation in the near-field approximation}

Now we compute the solution of the frequency-dependent radial Teukolsky equation (6.7) for the mode function $R$ in the potential region (near-field zone), characterized by

$$
x\left(r_{+}-r_{-}\right) \ll(\ell+1) / \omega .
$$

for a rotating black hole and perturbation of a generic spin $s$. We additionally expand over the small parameter $\omega M \sim \omega r_{s} \ll 1$. Using that $\omega a \leq \omega M \ll 1$, we can approximate $A=(\ell-s)(\ell+s+1)$ and hence the radial differential equation can be written in the following simple form [69, 70],

$$
\left[s^{2}+s-\ell^{2}-\ell+\frac{Q^{2}+i s Q(2 x+1)}{x(x+1)}+(s+1)(2 x+1) \frac{d}{d x}+x(x+1) \frac{d^{2}}{d x^{2}}\right] R(x)=0 .
$$

This is the same equation as (6.1), but with $\gamma m$ replaced by $Q$. Hence, the required solution is given by eq. (6.3) with $\gamma m \rightarrow Q$. If we now formally analytically continue this solution for $x>1$, we can obtain the following expression for response coefficients

$$
\begin{aligned}
k_{\ell m}^{(s) \mathrm{NF}} & =\frac{\Gamma(-2 \ell-1) \Gamma(\ell-s+1) \Gamma(\ell+2 i Q+1)}{\Gamma(-\ell-s) \Gamma(2 i Q-\ell) \Gamma(2 \ell+1)}\left(\frac{r_{+}-r_{-}}{r_{s}}\right)^{2 \ell+1} \\
& =(-1)^{s+1} \frac{i}{2}\left(m \chi-2 r_{+} \omega\right) \frac{(\ell+s) !(\ell-s) !}{(2 \ell) !(2 \ell+1) !} \prod_{n=1}^{\ell}\left[n^{2}\left(1-\chi^{2}\right)+\left(m \chi-2 r_{+} \omega\right)^{2}\right] \\
& =(-1)^{s+1} i r_{+}(m \Omega-\omega) \frac{(\ell+s) !(\ell-s) !}{(2 \ell) !(2 \ell+1) !} \prod_{n=1}^{\ell}\left[n^{2}\left(1-\chi^{2}\right)+4 r_{+}^{2}(m \Omega-\omega)^{2}\right] .
\end{aligned}
$$

where we used black hole's angular velocity $\Omega \equiv a /\left(r_{+}^{2}+a^{2}\right)=a /\left(2 M r_{+}\right)$. We can see that this expression is not invariant under time reversal transformations $\omega \rightarrow-\omega, m \rightarrow-m$, which implies that the near-field response is purely dissipative. However, this result is uncertain up to other frequency-dependent corrections. To estimate these corrections, let us use the perturbed angular eigenvalues (6.8) instead of the usual ones. We have:

$$
\tilde{\nu}=\ell+\Delta \ell=\ell+a \omega \frac{2 m s^{2}}{\ell(\ell+1)(2 \ell+1)}+\ldots
$$

Now we can easily find a solution to eq. (D.11) with $\ell$ replaced by $\nu$. It is given by

$$
\left.R\right|_{x \rightarrow \infty}=\text { const } \times x^{\tilde{\nu}}\left(1+x^{-2 \tilde{\nu}-1} \frac{\Gamma(-2 \tilde{\nu}-1) \Gamma(\tilde{\nu}-s+1) \Gamma(\nu+2 i Q+1)}{\Gamma(-\tilde{\nu}-s) \Gamma(2 i Q-\tilde{\nu}) \Gamma(2 \tilde{\nu}+1)}\right) .
$$

Therefore, the relevant response coefficient reads

$$
k_{\ell m}^{(s) \mathrm{NF}}=\frac{\Gamma(-2 \tilde{\nu}-1) \Gamma(\tilde{\nu}-s+1) \Gamma(\tilde{\nu}+2 i Q+1)}{\Gamma(-\tilde{\nu}-s) \Gamma(2 i Q-\tilde{\nu}) \Gamma(2 \tilde{\nu}+1)}\left(\frac{r_{s a}}{r_{s}}\right)^{2 \ell+1}(1-2 \Delta \ell \ln x),
$$

where the logarithm comes from the Taylor expansion of $x^{-2 \tilde{\nu}-1}$,

$$
x^{-2 \tilde{\nu}-1}=x^{-2 \ell-1}(1-2 \Delta \ell \ln x) .
$$


After some simplifications we obtain

$$
\begin{aligned}
k_{\ell m}^{(s) \mathrm{NF}}= & (-1)^{s+1} \sin (2 i Q \pi-\Delta \ell \pi) \frac{2 i Q}{\sin (2 i Q \pi)} \frac{(\ell-s) !(\ell+s) !}{2(2 \ell) !(2 \ell+1) !} \prod_{n=1}^{\ell}\left(n^{2}+4 Q^{2}\right) \\
& \times\left(r_{s a} / r_{s}\right)^{2 \ell+1}(1-2 \Delta \ell \ln x)+\mathcal{O}\left(\Delta \ell^{2}\right) .
\end{aligned}
$$

For $\Delta \ell=0$ the response coefficients reduce to eq. (D.12). However, we can see that the near field approximation misses $\mathcal{O}(\omega a)$ and $\mathcal{O}(\omega a \ln x)$ corrections. This can be confirmed by an explicit comparison with a solution obtained in a small-frequency expansion of the Teukolsky equation [71-74].

\section{D.3 Comparison with the low-frequency solution}

The systematic treatment of the Teukolsky equation in the low-frequency limit [71-74] gives a solution which is somewhat different from the near-field expression, cf. eq. (D.12) and eq. (6.16). Importantly, the relevant response coefficients are not purely imaginary in this case. To see this, let us expand eq. (6.14) to linear order in $\epsilon$, while keeping all powers of $\tilde{Q}$. Because of the presence of the simple pole at $\epsilon=0$, is important that we also expand the renormalized angular momentum $\nu=\ell+\Delta \ell$, where $\ell$ is an integer number satisfying $\ell \geq|s|$, and $\Delta \ell=\mathcal{O}\left(\epsilon^{2}\right)$. We have

$$
\begin{aligned}
\frac{\Gamma(-2 \nu-1)}{\Gamma(2 \nu+1)} & =\frac{1}{2 \Delta \ell(2 \ell+1) !(2 \ell) !}+\mathcal{O}\left(\epsilon^{0}\right), \\
\frac{\Gamma(\nu+1-i \epsilon-s)}{\Gamma(-\nu-s-i \epsilon)} & =(-1)^{\ell+s+1}(i \epsilon+\Delta \ell)(\ell-s) !(\ell+s) !+\mathcal{O}\left(\epsilon^{3}\right), \\
\frac{\Gamma(\nu+2 i \tilde{Q}+1)}{\Gamma(-\nu+2 i \tilde{Q})} & =(-1)^{\ell} \sin (2 i \tilde{Q} \pi-\Delta \ell \pi) \frac{2 i \tilde{Q}}{\sin (2 i \tilde{Q} \pi)} \prod_{n=1}^{\ell}\left(n^{2}+4 \tilde{Q}^{2}\right)+\mathcal{O}\left(\epsilon^{4}\right),
\end{aligned}
$$

This gives

$$
\begin{aligned}
\varkappa_{\nu m}^{(s)}= & {\left[\frac{-2 \tilde{Q} \epsilon}{\Delta \ell}+\frac{2 i \tilde{Q}}{\sinh (2 \tilde{Q} \pi)}(\sinh (2 \pi \tilde{Q})-\epsilon \pi \cosh (2 \pi \tilde{Q}))-\Delta \ell \frac{(2 \pi \tilde{Q}) \cosh (2 \pi \tilde{Q})}{\sinh (2 \pi \tilde{Q})}\right] } \\
& \times(-1)^{s+1} \frac{(\ell-s) !(\ell+s) !}{2(2 \ell+1) !(2 \ell) !}\left(\prod_{n=1}^{\ell}\left(n^{2}+4 \tilde{Q}^{2}\right)\right) .
\end{aligned}
$$

We see that our response coefficient has a pole at $\Delta \ell=0$. When we match the EFT result to the GR calculation, we use only finite parts, and hence this singular contribution can be ignored. ${ }^{19}$ However, it is important to note that this term also generates a finite 
logarithmic contribution,

$$
\begin{aligned}
\varkappa_{\nu m}^{(s)} x^{-2 \nu-1}= & \varkappa_{\nu m}^{(s)} x^{-2 \ell-1}\left(1-2 \Delta \ell \ln x+\mathcal{O}\left(\epsilon^{2}\right)\right) \\
= & x^{-2 \ell-1}\left[4 \tilde{Q} \epsilon \ln x+\frac{2 i \tilde{Q}}{\sinh (2 Q \pi)}(\sinh (2 \pi \tilde{Q})-\epsilon \pi \cosh (2 \pi \tilde{Q}))\right] \\
& \times(-1)^{s+1} \frac{(\ell-s) !(\ell+s) !}{2(2 \ell+1) !(2 \ell) !}\left(\prod_{n=1}^{\ell}\left(n^{2}+4 \tilde{Q}^{2}\right)\right)+\mathcal{O}\left(\epsilon^{2}\right) .
\end{aligned}
$$

Using that

$$
\sinh (2 \pi \tilde{Q})-\epsilon \pi \cosh (2 \pi \tilde{Q})=\sinh (2 \pi \tilde{Q}-\epsilon \pi)+\mathcal{O}\left(\epsilon^{2}\right),
$$

we obtain the first correction in eq. (6.16). We see that this expression coincidently matches the near-zone result eq. (D.12) at linear order in $\omega$ and zeroth order in $\omega \Omega$. However, the near-field approximation does not correctly capture $\mathcal{O}\left(\epsilon^{2}, \epsilon \Omega\right)$ corrections and their logarithmic running.

Extracting the other contributions from eq. (6.15) is straightforward. Collecting everything together we arrive at eq. (6.16).

\footnotetext{
${ }^{19}$ It is also worth stressing that the full GR solution is regular, the pole in eq. (D.18) in fact gets canceled by a similar singularity that is contained in the source series. To see this, we have to get back to the original solution (6.14) and regularize the hypergeometric function that is attached to the source solution $\propto x^{\nu}$ as follows
}

$$
\begin{aligned}
& { }_{2} F_{1}\left(-\nu+2 i \tilde{Q},-\nu+s+i \epsilon,-2 \nu,-x^{-1}\right) \\
& =(-x)^{-2 \ell-1} \frac{\Gamma(\ell+1+2 i \tilde{Q}) \Gamma(\ell+s+1+i \epsilon)}{\Gamma(2 \ell+2) \Gamma(-\ell+2 i \tilde{Q}) \Gamma(-\ell+s+i \epsilon)} \frac{-1}{(2 \ell) ! 2 \Delta \ell} \\
& \quad \times{ }_{2} F_{1}\left(\ell+1+2 i \tilde{Q}, \ell+s+1+i \epsilon, 2 \ell+2,-x^{-1}\right)+\mathcal{O}\left(\epsilon^{0}\right),
\end{aligned}
$$

which exactly cancels the divergence that we have encountered in the term $x^{-2 \nu-1} \varkappa_{\nu m}^{(s)}$, see eq. (D.18). 
Open Access. This article is distributed under the terms of the Creative Commons Attribution License (CC-BY 4.0), which permits any use, distribution and reproduction in any medium, provided the original author(s) and source are credited.

\section{References}

[1] A.E.H. Love, The Yielding of the Earth to Disturbing Forces, Proc. Roy. Soc. London Series A 82 (1909) 73.

[2] E.E. Flanagan and T. Hinderer, Constraining neutron star tidal Love numbers with gravitational wave detectors, Phys. Rev. D 77 (2008) 021502 [arXiv:0709.1915] [INSPIRE].

[3] V. Cardoso, E. Franzin, A. Maselli, P. Pani and G. Raposo, Testing strong-field gravity with tidal Love numbers, Phys. Rev. D 95 (2017) 084014 [Addendum ibid. 95 (2017) 089901] [arXiv: 1701.01116] [INSPIRE].

[4] W.D. Goldberger and I.Z. Rothstein, An Effective field theory of gravity for extended objects, Phys. Rev. D 73 (2006) 104029 [hep-th/0409156] [INSPIRE].

[5] R.A. Porto, The effective field theorist's approach to gravitational dynamics, Phys. Rept. 633 (2016) 1 [arXiv: 1601.04914] [INSPIRE].

[6] R.A. Porto, The Tune of Love and the Nature(ness) of Spacetime, Fortsch. Phys. 64 (2016) 723 [arXiv: 1606.08895 ] [INSPIRE].

[7] L. Hui, A. Joyce, R. Penco, L. Santoni and A.R. Solomon, Static response and Love numbers of Schwarzschild black holes, JCAP 04 (2021) 052 [arXiv: 2010.00593] [INSPIRE].

[8] H. Fang and G. Lovelace, Tidal coupling of a Schwarzschild black hole and circularly orbiting moon, Phys. Rev. D 72 (2005) 124016 [gr-qc/0505156] [InSPIRE].

[9] T. Damour and A. Nagar, Relativistic tidal properties of neutron stars, Phys. Rev. D 80 (2009) 084035 [arXiv:0906.0096] [INSPIRE].

[10] T. Binnington and E. Poisson, Relativistic theory of tidal Love numbers, Phys. Rev. D 80 (2009) 084018 [arXiv:0906.1366] [INSPIRE].

[11] B. Kol and M. Smolkin, Black hole stereotyping: Induced gravito-static polarization, JHEP 02 (2012) 010 [arXiv:1110.3764] [INSPIRE].

[12] V. Cardoso, L. Gualtieri and C.J. Moore, Gravitational waves and higher dimensions: Love numbers and Kaluza-Klein excitations, Phys. Rev. D 100 (2019) 124037 [arXiv:1910.09557] [INSPIRE].

[13] V. Cardoso, M. Kimura, A. Maselli and L. Senatore, Black Holes in an Effective Field Theory Extension of General Relativity, Phys. Rev. Lett. 121 (2018) 251105 [arXiv: 1808.08962] [INSPIRE].

[14] L. Bernard, Dipolar tidal effects in scalar-tensor theories, Phys. Rev. D 101 (2020) 021501 [arXiv: 1906.10735] [INSPIRE].

[15] N. Gürlebeck, No-hair theorem for Black Holes in Astrophysical Environments, Phys. Rev. Lett. 114 (2015) 151102 [arXiv: 1503.03240] [INSPIRE].

[16] R.P. Kerr, Gravitational field of a spinning mass as an example of algebraically special metrics, Phys. Rev. Lett. 11 (1963) 237 [InSPIRE].

[17] P. Pani, L. Gualtieri, A. Maselli and V. Ferrari, Tidal deformations of a spinning compact object, Phys. Rev. D 92 (2015) 024010 [arXiv: 1503.07365] [INSPIRE]. 
[18] P. Pani, L. Gualtieri and V. Ferrari, Tidal Love numbers of a slowly spinning neutron star, Phys. Rev. D 92 (2015) 124003 [arXiv:1509.02171] [InSPIRE].

[19] P. Landry and E. Poisson, Gravitomagnetic response of an irrotational body to an applied tidal field, Phys. Rev. D 91 (2015) 104026 [arXiv:1504.06606] [InSPIRE].

[20] P. Landry and E. Poisson, Tidal deformation of a slowly rotating material body. External metric, Phys. Rev. D 91 (2015) 104018 [arXiv:1503.07366] [INSPIRE].

[21] P. Landry, Tidal deformation of a slowly rotating material body: Interior metric and Love numbers, Phys. Rev. D 95 (2017) 124058 [arXiv:1703.08168] [INSPIRE].

[22] E. Poisson, Gravitomagnetic Love tensor of a slowly rotating body: post-Newtonian theory, Phys. Rev. D 102 (2020) 064059 [arXiv: 2007.01678] [INSPIRE].

[23] A. Le Tiec and M. Casals, Spinning Black Holes Fall in Love, Phys. Rev. Lett. 126 (2021) 131102 [arXiv: 2007.00214] [INSPIRE].

[24] S.E. Gralla, On the Ambiguity in Relativistic Tidal Deformability, Class. Quant. Grav. 35 (2018) 085002 [arXiv: 1710.11096] [INSPIRE].

[25] A. Le Tiec, M. Casals and E. Franzin, Tidal Love Numbers of Kerr Black Holes, Phys. Rev. D 103 (2021) 084021 [arXiv:2010.15795] [INSPIRE].

[26] H.S. Chia, Tidal Deformation and Dissipation of Rotating Black Holes, arXiv: 2010.07300 [INSPIRE].

[27] W.D. Goldberger, J. Li and I.Z. Rothstein, Non-conservative effects on Spinning Black Holes from World-Line Effective Field Theory, arXiv:2012.14869 [INSPIRE].

[28] E. Poisson, Compact body in a tidal environment: New types of relativistic Love numbers, and a post-Newtonian operational definition for tidally induced multipole moments, Phys. Rev. D 103 (2021) 064023 [arXiv: 2012.10184] [INSPIRE].

[29] E. Poisson and C.M. Will, Gravity: Newtonian, Post-Newtonian, Relativistic, Cambridge University Press (2014) [DOI].

[30] S. Chakrabarti, T. Delsate and J. Steinhoff, Effective action and linear response of compact objects in Newtonian gravity, Phys. Rev. D 88 (2013) 084038 [arXiv:1306.5820] [InSPIRE].

[31] S.J. Peale and P. Cassen, Contribution of tidal dissipation to lunar thermal history, Icarus 36 (1978) 245.

[32] P. Hut, Tidal evolution in close binary systems, Astron. Astrophys. 99 (1981) 126.

[33] M.E. Alexander, The Weak Friction Approximation and Tidal Evolution in Close Binary Systems, Astrophys. Space Sci. 23 (1973) 459.

[34] K.S. Thorne and J.B. Hartle, Laws of motion and precession for black holes and other bodies, Phys. Rev. D 31 (1984) 1815 [inSPIRE].

[35] J.M. Bardeen, W.H. Press and S.A. Teukolsky, Rotating black holes: Locally nonrotating frames, energy extraction, and scalar synchrotron radiation, Astrophys. J. 178 (1972) 347 [INSPIRE].

[36] P. Landry and E. Poisson, Dynamical response to a stationary tidal field, Phys. Rev. D 92 (2015) 124041 [arXiv:1510.09170] [INSPIRE].

[37] Y.B. Zel'Dovich, Generation of Waves by a Rotating Body, JETP Lett. 14 (1971) 180 [in russian, ZhETF Pisma Redaktsiiu 14 (1971) 270]. 
[38] I.D. Novikov and V.P. Frolov, Physics of black holes, Kluwer Academic, Dordrecht, The Netherlands (1989) [DOI] [INSPIRE].

[39] W.D. Goldberger and I.Z. Rothstein, Dissipative effects in the worldline approach to black hole dynamics, Phys. Rev. D 73 (2006) 104030 [hep-th/0511133] [InSPIRE].

[40] R.A. Porto, Absorption effects due to spin in the worldline approach to black hole dynamics, Phys. Rev. D 77 (2008) 064026 [arXiv:0710.5150] [InSPIRE].

[41] K.S. Thorne, Multipole Expansions of Gravitational Radiation, Rev. Mod. Phys. 52 (1980) 299 [INSPIRE].

[42] C.W. Misner, K.S. Thorne and J.A. Wheeler, Gravitation, W.H. Freeman, San Francisco U.S.A (1973).

[43] N. Yunes and J. Gonzalez, Metric of a tidally perturbed spinning black hole, Phys. Rev. D 73 (2006) 024010 [Erratum ibid. 89 (2014) 089902] [gr-qc/0510076] [INSPIRE].

[44] W. Israel, Event horizons in static vacuum space-times, Phys. Rev. 164 (1967) 1776 [INSPIRE].

[45] B. Carter, Axisymmetric Black Hole Has Only Two Degrees of Freedom, Phys. Rev. Lett. 26 (1971) 331 [INSPIRE].

[46] D.C. Robinson, Uniqueness of the Kerr black hole, Phys. Rev. Lett. 34 (1975) 905 [INSPIRE].

[47] S. Dubovsky, P. Tinyakov and M. Zaldarriaga, Bumpy black holes from spontaneous Lorentz violation, JHEP 11 (2007) 083 [arXiv:0706.0288] [INSPIRE].

[48] S.A. Teukolsky, Rotating black holes - separable wave equations for gravitational and electromagnetic perturbations, Phys. Rev. Lett. 29 (1972) 1114 [InSPIRE].

[49] S.A. Teukolsky, Perturbations of a rotating black hole. 1. Fundamental equations for gravitational electromagnetic and neutrino field perturbations, Astrophys. J. 185 (1973) 635 [INSPIRE].

[50] W.H. Press and S.A. Teukolsky, Perturbations of a Rotating Black Hole. II. Dynamical Stability of the Kerr Metric, Astrophys. J. 185 (1973) 649 [INSPIRE].

[51] S.A. Teukolsky and W.H. Press, Perturbations of a rotating black hole. III - Interaction of the hole with gravitational and electromagnet ic radiation, Astrophys. J. 193 (1974) 443 [INSPIRE].

[52] D.R. Brill, P.L. Chrzanowski, C. Martin Pereira, E.D. Fackerell and J.R. Ipser, Solution of the scalar wave equation in a kerr background by separation of variables, Phys. Rev. D 5 (1972) 1913 [INSPIRE].

[53] W.H. Press, Time Evolution of a Rotating Black Hole Immersed in a Static Scalar Field, Astrophys. J. 175 (1972) 243.

[54] J.F. Donoghue, M.M. Ivanov and A. Shkerin, EPFL Lectures on General Relativity as a Quantum Field Theory, arXiv:1702.00319 [INSPIRE].

[55] K. Martel and E. Poisson, Gravitational perturbations of the Schwarzschild spacetime: A Practical covariant and gauge-invariant formalism, Phys. Rev. D 71 (2005) 104003 [gr-qc/0502028] [INSPIRE].

[56] T. Kobayashi, H. Motohashi and T. Suyama, Black hole perturbation in the most general scalar-tensor theory with second-order field equations I: the odd-parity sector, Phys. Rev. D 85 (2012) 084025 [Erratum ibid. 96 (2017) 109903] [arXiv: 1202.4893] [INSPIRE]. 
[57] R.H. Boyer and R.W. Lindquist, Maximal analytic extension of the Kerr metric, J. Math. Phys. 8 (1967) 265 [INSPIRE].

[58] S. Chandrasekhar, The mathematical theory of black holes, Am. J. Phys. 53 (1985) 1013.

[59] A.A. Starobinskii, Amplification of waves during reflection from a rotating "black hole", JETP 37 (1973) 28.

[60] T. Damour and O.M. Lecian, On the gravitational polarizability of black holes, Phys. Rev. D 80 (2009) 044017 [arXiv: 0906. 3003] [INSPIRE].

[61] E. Newman and R. Penrose, An Approach to gravitational radiation by a method of spin coefficients, J. Math. Phys. 3 (1962) 566 [INSPIRE].

[62] E. Newman and R. Penrose, Errata: An Approach to Gravitational Radiation by a Method of Spin Coefficients, J. Math. Phys. 4 (1963) 998.

[63] T. Regge and J.A. Wheeler, Stability of a Schwarzschild singularity, Phys. Rev. 108 (1957) 1063 [INSPIRE].

[64] W. Kinnersley, Type D Vacuum Metrics, J. Math. Phys. 10 (1969) 1195 [inSPIRE].

[65] J. Bičák and L. Dvořák, Stationary electromagnetic fields around black holes. II. General solutions and the fields of some special sources near a Kerr black hole, Gen. Rel. Grav. 7 (1976) 959.

[66] J. Bicak and L. Dvorak, Stationary electromagnetic fields around black holes. I. General solutions and the fields of some special sources near a Schwarzschild black hole, Czech. J. Phys. 2 (1977) 127.

[67] J. Bicak and L. Dvorak, Stationary electromagnetic fields around black holes. III. General solutions and the fields of current loops near the Reissner-Nordström black hole, Phys. Rev. D 22 (1980) 2933 [INSPIRE].

[68] E. Poisson, Absorption of mass and angular momentum by a black hole: Time-domain formalisms for gravitational perturbations, and the small-hole / slow-motion approximation, Phys. Rev. D 70 (2004) 084044 [gr-qc/0407050] [INSPIRE].

[69] A.A. Starobinskii and S.M. Churilov, Amplification of electromagnetic and gravitational waves scattered by a rotating "black hole", JETP 38 (1974) 1.

[70] D.N. Page, Particle Emission Rates from a Black Hole: Massless Particles from an Uncharged, Nonrotating Hole, Phys. Rev. D 13 (1976) 198 [INSPIRE].

[71] S. Mano, H. Suzuki and E. Takasugi, Analytic solutions of the Teukolsky equation and their low frequency expansions, Prog. Theor. Phys. 95 (1996) 1079 [gr-qc/9603020] [InSPIRE].

[72] S. Mano, H. Suzuki and E. Takasugi, Analytic solutions of the Regge-Wheeler equation and the postMinkowskian expansion, Prog. Theor. Phys. 96 (1996) 549 [gr-qc/9605057] [INSPIRE].

[73] S. Mano and E. Takasugi, Analytic solutions of the Teukolsky equation and their properties, Prog. Theor. Phys. 97 (1997) 213 [gr-qc/9611014] [InSPIRE].

[74] M. Sasaki and H. Tagoshi, Analytic black hole perturbation approach to gravitational radiation, Living Rev. Rel. 6 (2003) 6 [gr-qc/0306120] [INSPIRE].

[75] S. Chakrabarti, T. Delsate and J. Steinhoff, New perspectives on neutron star and black hole spectroscopy and dynamic tides, arXiv:1304.2228 [INSPIRE]. 
[76] V.P. Frolov and D. Kubiznak, Higher-Dimensional Black Holes: Hidden Symmetries and Separation of Variables, Class. Quant. Grav. 25 (2008) 154005 [arXiv:0802.0322] [InSPIRE].

[77] M.M. Caldarelli, R. Emparan and B. Van Pol, Higher-dimensional Rotating Charged Black Holes, JHEP 04 (2011) 013 [arXiv: 1012.4517] [InSPIRE].

[78] E.T. Newman, R. Couch, K. Chinnapared, A. Exton, A. Prakash and R. Torrence, Metric of a Rotating, Charged Mass, J. Math. Phys. 6 (1965) 918 [InSPIRE].

[79] V.D. Sandberg, Tensor spherical harmonics on $S^{2}$ and $S^{3}$ as eigenvalue problems, J. Math. Phys. 19 (1978) 2441. 